\title{
THE ECB'S NEW
} MULTI-COUNTRY MODEL FOR THE

\section{EURO AREA}

\section{NMCM - SIMULATED \\ WITH RATIONAL EXPECTATIONS}

by Alistair Dieppe, Alberto González Pandiella and Alpo Willman 


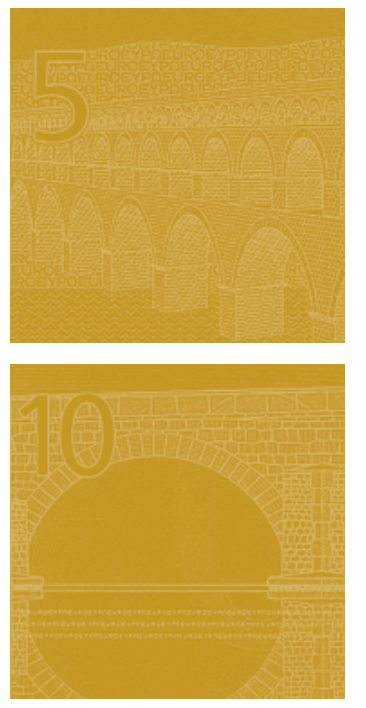

\section{WORKING PAPER SERIES}

NO I3I5 / APRIL 20II

\section{THE ECB'S NEW MULTI-COUNTRY MODEL FOR THE EURO AREA} NMCM - SIMULATED WITH
RATIONAL EXPECTATIONS '

by Alistair Dieppe, Alberto González Pandiella and Alpo Willman ${ }^{2}$

NOTE: This Working Paper should not be reported as representing the views of the European Central Bank (ECB).

The views expressed are those of the authors and do not necessarily reflect those of the ECB.

This paper can be downloaded without charge from http://www.ecb.europa.eu or from the Social Science Research Network electronic library at http://ssrn.com/abstract_id= 1788986 .

I Professor Stephan Hall acted as a consultant. Tommaso Aquilante, Thomas Kostka and Vladimir Georgescu provided valuable research assistance. We have also benefited from valuable input from Julian Morgan, Günter Coenen, Geoff Kenny,

Jérôme Henry, Elena Angelini, Pascal Jacquinot, Peter McAdam, Tohmas Karlsson as well as comments during presentations to the Eurosystem. All errors are our responsibility. 2 All authors: European Central Bank, Kaiserstrasse 29, D-603II Frankfurt am Main, Germany; e-mails: alistair.dieppe@ecb.europa.eu; alberto.gonzalez_pandiella@ecb.europa.eu; 


\section{(c) European Central Bank, 2011}

\section{Address}

Kaiserstrasse 29

60311 Frankfurt am Main, Germany

\section{Postal address}

Postfach 160319

60066 Frankfurt am Main, Germany

\section{Telephone}

+496913440

\section{Internet}

http://www.ecb.europa.eu

Fax

+496913446000

All rights reserved.

Any reproduction, publication and reprint in the form of a different publication, whether printed or produced electronically, in whole or in part, is permitted only with the explicit written authorisation of the ECB or the authors.

Information on all of the papers published in the ECB Working Paper Series can be found on the ECB's website, http://www. ecb.europa.eu/pub/scientific/wps/date/ html/index.en.html

ISSN 1725-2806 (online) 


\section{CONTENTS}

Abstract

Non technical summary

1 Introduction

2 Overview of the model framework

3 Consumer behaviour

3.1 The life-time budget constraint under uncertain life-time, uncertain labour income and front loaded information

3.2 The optimal consumption rule and the aggregate consumption function

4 Firm behaviour

4.1 Output, costs and dividends

4.2 Three-valued state dependent arrow-calvo signal

4.3 The first order conditions of the profit maximising firmdynamic euler conditions

4.4 Functional forms of the factor adjustment costs and the efficient to normal hours wage premium

4.5 Dynamic factor demand equations

4.6 New keynesian phillips-curve for prices

5 Union behaviour: wage setting

5.1 Calvo staggering wage inflation

5.2 The frictionless wage rate

6 The foreign trade block

6.1 Exports and export prices

6.2 Imports and import prices

7 Production function and the medium-run supply system

7.1 The normalised CES production function

7.2 The supply system

8 Estimation of the model

8.1 Data for estimation

8.2 Estimation of the supply system

8.3 Dynamic equation estimation

8.4 Households

8.5 External sector behaviour

8.6 Government, central banks and financial markets
9 Rational expectations 45

10 Indicative simulations 46

10.1 Short-term interest rate shock 47

10.2 Permanent government expenditure shock

10.3 Technology shock

10.4 Permanent exchange rate shock

10.5 Permanent world demand shock

10.6 Pre-announced (anticipated) shocks

10.7 Unannounced (unanticipated) change in policy

11 Conclusions 52

12 References 53

Appendix A. Instruments and normalisation 58 for some key equations

Appendix B. Figures

61 


\begin{abstract}
The model presented here is a New estimated medium-scale Multi-Country Model (NMCM) which covers the five largest euro area countries and is used for forecasting and scenarios analysis at the European Central Bank. The model has a tight theoretical structure which allows for non-unitary elasticity of substitution, non-constant augmenting technical progress and heterogeneous sectors with differentiated price and income elastiticites of demand across sectors. Furthermore, it has the explicit inclusion of expectations on the basis of three optimising private sector decision making units: i.e. firms, trade unions and households, where output is in the short run demand-determined and monopolistically competing firms set prices and factor demands. Labour is indivisible and monopoly-unions set wages and households make consumption/saving decisions.

We assume agents optimise under limited information where each agent knows only the parameters related to his/her optimization problem. Therefore we estimate with GMM, which implicitly assumes limited information boundedly rational expectations. In this paper we provide some simulation results under the assumption of model-consistent rational expectations, we show that there is some heterogeneity across countries and that the reactions of the economies to shocks depends strongly on whether the shocks are pre-announced, announced and credible or unannounced and uncredible.
\end{abstract}

Keywords: Macro model, Open-economy macroeconomics, Rational expectations

JEL Classification: C51, C6, E5 


\section{Non technical summary}

The model presented here is a new estimated medium-scale Multi-Country Model (NMCM) which covers the 5 largest euro area countries (Germany, France, Italy, Spain and the Netherlands) and can be used either on a single country basis or as a linked euro area multi-country model. The model is the main macroeconomic tool used to build the European Central Bank staff projections for individual euro area countries in the context of the Eurosystem Macroeconomic Projection exercises (see ECB 2001). Given the need by central bankers to analyse the interaction of a large set of variables in the economy, small models, by their nature, have limited policy use. Therefore, we have aimed to build a model with both economic coherence, that matches the key characteristics of the data and that is useful to build projections and to analyse policy issues via scenario analysis. The NMCM will enable us to analyse cross-country interaction of fiscal, monetary, and competitiveness issues, just to name a few of the possibilities.

The model has firm micro-economic foundations with the theoretic core of the model containing one exportable domestic good and one imported good. All central behavioural relations are based on the optimisation behaviour of three private sector decision making units (i.e. households, labour unions and firms) and the reaction functions of the government sector and the central bank. Expectation formation is treated explicitly and the model can be characterised as a micro-founded New Keynesian model. Although in all these respects the NMCM closely resembles DSGE models, its differences to them are also clear, especially, in the following two ways. Firstly, the model is specified to track both the short-run dynamics around the medium-run equilibrium and also the deviations of the medium-run developments from the balanced-growth-path. This is executed by a careful modelling of the supply-side allowing non-unitary elasticity of substitution between labour and capital and quite general factor augmenting technical progress accounting for cross-equation restrictions. Secondly, the DSGE assumption that all optimizing agents know fully the whole structure and all parameter values of the model including the stochastic processes generating exogenous shocks is replaced by bounded rationality. Thus, each agent knows only the parameters related to her optimization problem but does not need to know the rest of the model, i.e. other agent's optimising problems, nor, more importantly, the stochastic processes driving the variables predetermined to them. This modelling strategy allows the use of the model under rational expectations and to flexibly vary the degree at which the exact stochastic nature of the shock is correctly anticipated, as well as to extend the framework further to account for boundedly rational learning, where the true stochastic nature of the shock is gradually learned. However, we reserve the detailed presentation of this important extension for an accompanying separate report, see Dieppe et. al (2011).

Our modelling approach has been, within the constraints of the common theoretic setting, to allow the data to determine estimated parameter values, with only a limited amount of constraints. Therefore, we estimate the supply side by non-linear SUR which allows for cross-equation restrictions in the medium-run, and the dynamic equations are estimated by GMM, which implicitly assumes limited information bounded rational expectations.

Our indicative simulations are undertaken with agents' expectations based on rational, model consistent expectations. These simulations suggest the impulse responses of the model to exogenous shocks are plausible with cross-country differences reflecting estimated country heterogeneity. Furthermore, under our framework, the reaction of the economy to shocks depends strongly on whether it is pre-announced and/or correctly anticipated (perfectly informed), or unannounced and uncredible (imperfectly informed). With forward-looking rational expectations model responses to shocks are quick and the model solution converges fast to the long-run equilibrium. However, the shock response effects become more delayed and comparable to those in many DSGE models, if the shock generating processes are specified to follow some simple AR process, as it is the practice in the DSGE models. 


\section{Introduction}

The model presented here is a New estimated medium-scale Multi-Country Model (NMCM) developed at the European Central Bank. It covers the 5 biggest euro area countries (Germany, France, Italy, Spain and the Netherlands) and can be used either on a single country basis or as a linked euro area multi-country model. This model is the main country macroeconomic tool used at the ECB in the context of the Eurosystem Macroeconomic Projection exercises (see ECB 2001). Given the need by central bankers to analyse the interaction of a large set of variables in the economy, small models, by their nature, have limited policy use. Therefore, we have aimed to build a model with both economic coherence, that matches the key characteristics of the data and that is useful to build projections and to analyse policy issues via scenario analysis. The NMCM will enable us to analyse cross-country interaction of fiscal, monetary, and competitiveness issues, just to name a few of the possibilities.

The model has firm micro-economic foundations with the theoretic core of the model containing one exportable domestic good and one imported good. All central behavioural relations are based on the optimisation behaviour of three private sector decision making units (i.e. households, labour unions and firms) and the reaction functions of the government sector and the central bank. Expectation formation is treated explicitly and the model can be characterised as a micro-founded New Keynesian model. Although in all these respects the NMCM closely resembles DSGE models, its differences to them are also clear, especially, in the following two ways. Firstly, the model is specified to track both the short-run dynamics around the medium-run equilibrium and also the deviations of the medium-run developments from the balanced-growth-path. This is executed by a careful modelling of the supply-side allowing non-unitary elasticity of substitution between labour and capital and quite general factor augmenting technical progress accounting for cross-equation restrictions. Secondly, the DSGE assumption that all optimizing agents know fully the whole structure and all parameter values of the model including the stochastic processes generating exogenous shocks is replaced by bounded rationality. Thus, each agent knows only the parameters related to her optimization problem but does not need to know the rest of the model, i.e. other agent's optimising problems, nor, more importantly, the stochastic processes driving the variables predetermined to them. This modelling strategy allows the use of the model under rational expectations and to flexibly vary the degree at which the exact stochastic nature of the shock is correctly anticipated, as well as the use of the model under boundedly rational learning, where the true stochastic nature of the shock is gradually learned.

Our modelling approach has been, within the constraints of the common theoretic setting, to allow the data to determine estimated parameter values, with only a limited amount of constraints. Therefore, we estimate the supply side by non-linear SUR which allows for cross-equation restrictions in the medium-run, and the dynamic equations are estimated by GMM, which implicitly assumes limited information bounded rational expectations.

This report is the first in the series of reports presenting the NMCM. It presents the common theoretical structure of individual country blocks, their estimation and the simulation properties of each country block on a single county basis under rational model consistent expectations. The second report will present the boundedly rational learning version of the model and studies the behaviour of the model under alternative assumptions concerning expectation formation. The third report will present the linked NMCM model which includes a sixth block covering the rest of the euro area and studies the model properties under the common monetary and exchange rate policy and compares them to the simulation results done on a single country basis. 


\section{Overview of the Model Framework}

This section gives a concise overview of the main features of the theoretical model. The detailed theoretical framework and the derivation of the estimated specifications are presented in the following sections.

The theoretical core of the model consists of three optimising private sector decision making units, namely utility maximising households, profit maximising firms and trade unions. Monopolistically competing firms set prices, inventories, fixed investment and employment under the assumptions of indivisible labour. Output is in the short run demand-determined. Overlapping generation households make consumption/saving decisions and monopoly unions set wages by minimising the quadratic loss function under the staggered wage adjustment assumption. There are two types of trade unions: utilitarian and non-utilitarian. In a right-to-manage framework, the utilitarian unions maximize the member households' utility allowing the employment rate to vary. The non-utilitarian unions target the employment rate via real wage demands. The share of utilitarian and non-utilitarian unions can be flexibly changed, depending for instance on the purpose of the simulation exercise. This is useful for model diagnosis because a non-utilitarian trade union alternative implies a constant long-run NAIRU, while the utilitarian alternative implies that the NAIRU reacts to permanent shocks. In addition, the utilitarian union alternative renders the model suitable for welfare analysis of economic policy.

As the available data do not disaggregate government into separate institutional sectors, the theoretical core of the model assumes a single domestic good produced by an aggregated production function with total employment and total capital stock as inputs. Hence, the optimisation framework derives the "true" behavioural relations for total employment, investment, private consumption and corresponding deflators and factor prices. For forecasting purpose, however, the accounting framework of the model is markedly more disaggregated, but with no feed-back effects on the longer-run adjustment dynamics of the model.

The real world data which we have to confront stands in stark contrast to the predictions of many simple macroeconomic models. In particular, a simple model with a balanced growth path (BGP), as typically adopted e.g. by DSGE and other models related to the real business cycle (RBC) paradigm, would predict that the GDP-shares of capital, labour and total factor income as well as the capital-output ratio are stationary. In the real data for our five countries this is clearly not the case. Therefore, as discussed by McAdam and Willman (2008), Solow (2000) and Blanchard (1997), we adopt a medium-run view regarding the underlying "trend" developments of our data in the sample period. Accordingly, the medium run developments, towards which the short-run dynamics converges, are allowed to deviate from the BGP. However, this view does not exclude the possibility that many processes, which from a medium run perspective may be advisable to treat as exogenous, are from the very long-run perspective endogenous and drive the medium run development eventually to converge to the BGP. Acemoglu (2002, 2003) gives an excellent example by showing that while technical progress is necessarily labour-augmented along the BGP, it may become capitalaugmented in periods of transition reflecting the interplay of innovation activities, factor intensities and profitability. Given a below-unitary substitution elasticity, this pattern promotes the asymptotic stability of income shares while allowing them to fluctuate in the medium run. Accordingly, we allow non-unitary elasticity of substitution, non-constant augmenting technical progress and heterogeneous sectors with differentiated price and income elasticities of demand across sectors. We achieve this following McAdam and Willman (2008 and 2010), by assuming a normalised Constant Elasticity of Substitution (CES) production function and augmented technical progress modelled in terms of BoxCox functions (Box and Cox (1964)). The assumption of heterogeneous sectors, in turn, allows a non-stationary aggregate mark-up that can also be observed in the data. 
In addition to the relaxations concerning the medium-run development, the optimisation frameworks of agents contain a lot of frictional elements which are needed to explain realistically the observed stylised short-run features. Labour is indivisible with important implications for the behaviour of optimising agents. Regarding households' utility maximisation problem, the indivisibility assumption simplifies the analysis, because the labour supply adjusts to the demand for labour conditional on the wage contract set by unions maximising either the utility of member households or targeting the warranted wage rate consistent with a desired employment rate. The basic framework in household's utility maximisation is Blanchard's (1985) overlapping generation framework of perpetual youth that implies that the discount rate of the optimizing households is modified to include the probability of dying. This framework is especially useful in a small-open economy environment as it suffices to ensure that agents do not borrow indefinitely at the fixed external rate of interest. Hence, the long run stability of foreign assets is attained without resorting to the somewhat ad-hoc assumption of the foreign debt elastic interest rate or the endogenous discount factor, as is typically postulated in the infinite horizon DSGE models, see more closely Schmitt-Grohé and Uribe (2003). We extend Blanchard's overlapping generations framework in many ways with the final aim to improve its ability to trace the observed hump-shaped response pattern of consumption to shocks. Firstly we incorporate income uncertainty into the utility maximization framework. To do it in a tractable way, we apply a two-stage approach, see Willman (2007). In the first stage, the consumer evaluates her risk-adjusted non-human and human wealth conditional on uncertain lifespan and labour income. Thereafter, in the second stage, conditional on the risk adjusted life-time resource constraint, the consumer is assumed to determine her optimal planned path of consumption. This approach gives a closed form consumption function with precautionary saving depending positively on the income risk and death probability. The explicit treatment of wealth allows us also to account for asset price (i.e. stock and house prices) effects on the perceived wealth relevant for consumption decisions. Further, the assumption of imperfect-front loaded information on future income realisations changes also the weight structure in defining the present value of the expected income stream to be more front-loaded and increases the dependency of consumption on current income. Hence, we do not need to split households artificially into Ricardian utility maximisers and non-optimising income-constrained consumers to introduce the observed strong dependency of consumption on current income. Instead, all consumers are non-Ricardian utility maximisers. Finally, the assumption of habit persistency introduces the dependency of current consumption on lagged consumption and is able to generate a hump-shaped response profile of consumption to shocks.

In the profit maximising framework of the firm the assumptions of indivisible labour, adjustment costs with respect to number of workers and convex costs with respect to work intensity introduce the discrepancy between paid hours and efficient hours. This explains the observed pro-cyclicality in labour productivity, when labour input is measured in heads or paid hours. It also introduces the ratio of efficient hours (per worker) to normal hours into an argument of optimal price setting on top of the conventionally defined marginal cost of labour. The price setting of firms and the wage setting of unions are staggered with three-valued Calvo-signal, McAdam and Willman (2010). Part of firms (unions) keep prices (wages) fixed, another part changes prices (wages) following backward-looking rule and the rest set them optimally based on intertemporal optimization.

To capture the observed inertia in capital formation, capital stock and its rate of change are coupled with adjustment costs. Also the ceiling to debt-financing ratio and the irreversibility of investment are imposed with implications for the rate at which the future is discounted and for the sensitivity of investment with respect to the user cost. Regarding the stock formation, firms minimise quadratic losses induced by the deviations of inventories, on the one hand, and production, on the other hand, from their respective target levels related to the level of production implied by the production function, 
when existing inputs are utilised at their normal (cost minimising) rates.

The steady state form of the first-order conditions of profit maximising firms and the utility of member households maximising unions imply the 5-equation medium-run supply system that allows a consistent two-step estimation of the underlying deep parameters of the model. As the supply system contains cross-equation parameter constrains, it is estimated with the method of non-linear SUR that León-Ledesma et al. (2010) have proven to be a very efficient estimation approach outperforming all single-equation methods. This system defines all parameters related to technology, production function and the mark-up, allowing to define optimal frictionless prices, wages, labour input, marginal cost and marginal product concepts needed in estimating in the second stage the dynamic first order optimisation conditions of firms and unions.

All dynamic equations containing the leads of variables are estimated by the generalised method of moment (GMM) that is compatible with the assumption of bounded rationality.

All euro area countries are open economies and, therefore, also in our theoretic single domestic good framework, a part of output is exported. However, firms face separate demand functions in domestic and export markets leading to the pricing to market behaviour. This effectively separates the optimal price setting of exports from the rest of the firm's optimisation problem. We assume that the volume of exports is determined by the almost ideal demand system (AIDS) function. The advantage of this functional form compared to the conventional iso-elastic form is that now, compatibly with empirical evidence, the foreign competitors' price affect optimal export-price setting. The export demand and the optimal price setting result in a two-equation system with cross-equation parameter constraints. This allows a model consistent way to estimate the price elasticity of export demand. Import side of the model is more conventional being determined by domestic demand and the relative price of imports to domestic good.

In the following sections we go into more detail on the framework of the model, starting with consumer behaviour in section 3, then firm and union behaviour in sections 4 and 5 before turning to the foreign trade block in section 6 . Finally we specify the production function and the medium-run supply system in section 7 . Those who want to skip the derivation can go directly to section 8 where we provide the estimates of the key equations in the model.

\section{Consumer behaviour}

Following Willman (2007) we derive a solved-out forward-looking aggregate consumption function with constant relative risk aversion (CRRA) preferences that is able to capture precautionary saving as a response to income uncertainty. Although, this consumption function is derived in a unified optimization framework, it results in non-Ricardian behaviour without dividing consumers into optimizing and non-optimizing sub-groups.

We assume that in the mental frames of consumers the optimization problem is solved sequentially rather than simultaneously, which is an implicit assumption in the dynamic programming approach ${ }^{1}$. This assumption is needed to have a closed form solution. In the first stage the consumer is assumed to evaluate her risk-adjusted non-human and human wealth conditional on uncertain lifespan and labour income. Thereafter, in the second stage, conditional on the risk adjusted life-time resource constraint, the consumer determines her optimal planned path of consumption. ${ }^{2}$

\footnotetext{
${ }^{1}$ The sequential approach to the consumer's optimization problem can also be thought as a simplifying rule in line with bounded rationality. For instance, Akerlof and Yellen (1985), Akerlof (2002), Thaler (1994, 2000) and Gabaix and Laibson (2000) have argued that, rather than being fully rational, agents may use nearly rational decision strategies summarising information and making choices based on simplified mental frames.

${ }^{2} \mathrm{~A}$ familiar example of sequential approach elsewhere in economics is the analysis of the portfolio selection problem,
} 
The second key assumption concerns the information that consumers have on future income innovations. More explicitly, we assume that the amount of period-specific information is front-loaded so that, with the lengthening of the projection horizon, expected income changes converge to those implied by some stochastic time-series process, which for simplicity is assumed to be a random walk.

Furthermore, we connect this framework to Blanchard's (1985) overlapping generation model with the positive probability of death and complement it to include habit formation. Habit formation is the way our framework accounts for the observed excess sensitivity of aggregate consumption with respect to lagged information. After aggregating, we end up with a dynamic consumption function where current consumption depends on one period lead and lag of consumption as well as on fundamental variables, i.e. on real non-human wealth, current-period real labour income and the real interest rate as well as on the determinants of time-varying precautionary saving. In the following sections we discuss the effects of income uncertainty, front-loaded information and habit persistence on the life-time budget constraint and then derive the solved-out aggregate consumption function.

\subsection{The life-time budget constraint under uncertain life-time, uncertain labour income and front loaded information}

In this section we derive the risk adjusted expected life-time wealth of a consumer. We start by introducing income uncertainty into the overlapping generation framework. Thereafter, we define explicitly the income generating process, its front loaded information content and derive the expected risk-adjusted wealth. Finally we modify the implied life-time budget constraint accounting for habit formation.

\subsubsection{Risk adjusted life-time wealth}

As in Blanchard (1985), each agent faces a constant probability of death $\pi$. Agents are selfish in a sense that they have no bequest motive. To remove involuntary bequests resulting from uncertainty about death, access to fair annuity markets are assumed, i.e. agents can contract with life insurance companies to receive a payment contingent on their death. Denoting by $V_{k, t}$ the end of period $t$ real non-human wealth of an agent born in the beginning of period $k$, agents in the $k$-th cohort may contract to receive a payment $(\pi /(1-\pi)) V_{k, t-1}$, if they do not die and pay $V_{k, t-1}$, if they do die in the beginning of period $t$. Hence, no bequests to younger generations are left. Now the dynamic budget constraint of identical individuals in the $k$-th cohort is:

$$
V_{k, t}=\left(1+r_{t}\right)\left(\frac{V_{k, t-1}}{1-\pi}+y_{k, t}-c_{k, t}\right)
$$

In (1) $y_{k, t}$ is labour income (net of taxes minus transfers), $c_{k, t}$ is consumption and $r_{t}$ is the real interest rate in period $t$. For each consumer the dynamic budget constraint (1) implies the following (ex-post) intertemporal budget constraint:

$$
\sum_{i=0}^{\infty} R_{t, t+i}(1-\pi)^{i} c_{k, t+i}=\frac{V_{k, t-1}}{1-\pi}+\sum_{i=0}^{\infty} R_{t, t+i}(1-\pi)^{i} y_{k, t+i}
$$

where $R_{t, t+i}=\left(1+r_{t}\right) / \prod_{j=0}^{i}\left(1+r_{t+j}\right)$.

We treat the real interest rate deterministic and, for notational reasons, constant in our theoretical analysis. Accordingly $R_{t, t+i}=R^{i}$ when $i \geq 1$. However, to allow the separation of the (constant) where the choice of optimal portfolio is separated from the rest of the household's optimization problem (Markowitz, 1952). 
equilibrium long-term interest rate from the short-term rate in our analysis, we denote $(1-\bar{R})^{-1}=$ $\sum_{0}^{\infty} R^{i}\left(=\sum_{0}^{\infty} R_{t, t+i}\right)$.

Now, with an uncertain income stream (2) can be written in the form:

$$
\begin{gathered}
\sum_{i=0}^{\infty} R^{i}(1-\pi)^{i} c_{k, t+i}=\frac{V_{k, t-1}}{1-\pi}+\underbrace{\sum_{i=0}^{\infty} R^{i}(1-\pi)^{i} E_{t} y_{k, t+i}}_{H_{k, t}^{E}} \\
+\sum_{i=1}^{\infty} R^{i}(1-\pi)^{i} v_{k, t+i}=W_{k, t}
\end{gathered}
$$

where $v_{k, t+i}=y_{k, t+i}-E_{t} y_{k, t+i}, E_{t}$ is the expectation operator, $H_{k, t}^{E}\left(=E_{t} H_{k, t}\right)$ is expected human wealth and $W_{k, t}$ total (ex post) wealth. We see that (3) contains also the present value of stochastic income innovations $v_{k, t+i}$, the size of which is not known ex ante. However, a deterministic riskadjusted life time-wealth equivalent to the ex-ante uncertain wealth can be derived, if the stochastic properties of the income generation process are known. In addition, this risk-adjusted wealth is separable to expected wealth component $\left(V_{k, t-1} /(1-\pi)+H_{k, t}^{E}\right)$ and the risk-adjustment component, i.e. the present value of the planned precautionary saving, if the functional form of the utility function is CRRA. However, before showing this we discuss the information set available to the consumer concerning her future income realisations. In that regard we deviate from the convention that the consumer has either perfect foresight or that, besides the knowledge of the stochastic properties of the past income stream, the consumer has no additional information on her future income realisations. We assume that the consumer may have a lot of period-specific information concerning future income changes although this information may be strongly front-loaded.

\subsubsection{Front loaded information and the expected human wealth}

Denote by $x_{k, t+i}$ the set of the ex-ante possible outcomes of the future realizations of income changes $\Delta y_{k, t+i}$. Assume that there are two kinds of information concerning future income realizations, i.e. information on the stochastic properties of the past income stream, which for simplicity is assumed to follow a random walk, and period-specific information on future income realizations. The amount of period-specific information concerning the period $t+i$ is measured by the parameter $\gamma_{t+i}$, which can range from zero to one. The closer to zero (one) $\gamma_{t+i}$, the less (more) the amount of periodspecific information is available on period $t$ concerning the income realization on period $t+i$. Denote by $f\left(x_{k, t+i} \mid \gamma_{t+i}\right)$ the conditional probability density function containing both time series and period specific information available to the consumer on period $t$ about future realization $\Delta y_{k, t+i}$. It is apparent that the distribution function $f\left(\cdot \mid \gamma_{t+i}\right)$ is asymmetric being the more skewed towards the actual realisation, the closer to unity $\gamma_{t+i}$ is. As suggested by Marron and Wand (1992) the asymmetric density function $f\left(\cdot \mid \gamma_{t+i}\right)$ can quite flexibly be presented as a mixture of two normally distributed density function as follows:

$$
f\left(x_{k, t+i} \mid \gamma_{t+i}\right)=\gamma_{t+i} g\left(x_{k, t+i}\right)+\left(1-\gamma_{t+i}\right) h\left(x_{k, t+i}\right)
$$

where the density function $g\left(x_{k, t+i}\right)$, related to the period specific information, is normally distributed with mean $\Delta y_{k, t+i}$ and variance $\left(1-\gamma_{t+i}\right) \sigma_{g}^{2}$ and the density function $h\left(x_{k, t+i}\right)$, related to the random walk realizations, is normally distributed with mean zero and variance $\sigma_{h}^{2}$. The weights 
$\gamma_{t+i}$ and $1-\gamma_{t+i}$ show the shares of probability mass related to period-specific information and to the random-walk process respectively. Under that assumption it is straightforward to show that the mean and variance of the function are:

$$
\begin{gathered}
\int_{-\infty}^{\infty} x_{k, t+i} f\left(x_{k, t+i} \mid \gamma_{t+i}\right) d x_{k, t+i}=\gamma_{t+i} \Delta y_{k, t+i} \\
\int_{-\infty}^{\infty}\left(x_{k, t+i}-\gamma_{t+i}\right)^{2} f\left(x_{k, t+i} \mid \gamma_{t+i}\right) d x_{k, t+i} \\
=1-\gamma_{t+i}\left[\sigma_{h}^{2}+\gamma_{t+i}\left(\sigma_{g}^{2}+\Delta y_{k, t+i}\right)\right]
\end{gathered}
$$

We see that, when $\gamma_{t+i} \rightarrow 1$, then the mean of $f\left(\cdot \mid \gamma_{t+i}\right)$, i.e. the expected income realisation, approaches to $\Delta y_{k, t+i}$ and the variance to zero, i.e. to the limiting case of perfect foresight. Correspondingly, when $\gamma_{t+i} \rightarrow 0$, then $f\left(\cdot \mid \gamma_{t+i}\right)$ coincides with $h(\cdot)$ with zero mean and variance $\sigma_{h}^{2}$.

To be empirically applicable, one additional assumption concerning the distribution of the periodspecific information over the planning horizon is required. For that purpose, it is natural to assume that the information content is wider concerning income changes in the near future than regarding longer planning horizons. To be more explicit we assume that the information parameter $\gamma_{t+i}$ is determined by the following simple process $\gamma_{t+i}=\gamma^{i}$. Now on the basis of (6) $E_{t} y_{k, t+i}=y_{k, t}+\sum_{j=1}^{i} \gamma^{j} \Delta y_{k, t+j}$ and, hence, in (3) expected human wealth can be defined in terms of future realisations as follows:

$$
\begin{aligned}
H_{k, t}^{E} & =\sum_{i=0}^{\infty} R^{i}(1-\pi)^{i}\left(y_{k, t}+\sum_{j=1}^{i} \gamma^{j} \Delta y_{k, t+j}\right) \\
& =\frac{1-(1-\pi) \bar{R} \gamma}{1-(1-\pi) \bar{R}} \sum_{i=0}^{\infty}[R(1-\pi) \gamma]^{i} y_{k, t+i}
\end{aligned}
$$

The important property of relation (7) is that the average size of expected human wealth $H_{k, t}^{E}$ is practically unaffected by the size of the information parameter $\gamma$. This results from the fact that with $\bar{R}=R$ the term:

$$
\frac{1-(1-\pi) \bar{R} \gamma}{1-(1-\pi) \bar{R}} \sum_{i=0}^{\infty}[R(1-\pi) \gamma]^{i}=1 /[1-(1-\pi) R]
$$

which is independent from $\gamma$. Therefore, while a decrease in $\gamma$ reduces the size of the discounted income term $\sum_{j=1}^{i}[R(1-\pi) \gamma]^{j} y_{k, t+j}$ and makes the dependency of $H_{k, t}^{E}$ from future income stream more front loaded, it also increases the size of the scaling factor $\frac{1-(1-\pi) \bar{R} \gamma}{1-(1-\pi) \bar{R}}$, which compensates the effect of $\gamma$ on the discounted income term.

\subsubsection{Expected risk-adjusted wealth}

We assume that, in making risk adjustment to her uncertain expected life-time wealth the consumer applies the conventional Arrow-Pratt approach to risk, i.e. the consumer defines the deterministic equivalent for which she would be willing to exchange her expected, but risky, life-time wealth. However, in deriving the relevant risk adjustment to the wealth it matters how long the consumer expects her current consumption plan to remain relevant without a major revision. If the consumer in her mental accounting framework thinks that after having maximized her intertemporal utility in the beginning of current period she needs never again maximize her utility, then the uncertainty measure relevant for risk adjustment would be the variance of the discounted stochastic income-innovation stream $\sum_{i=1}^{\infty}[R(1-\pi)]^{i} v_{k, t+i}$. However, if the consumer expects that she needs to repeat her utility maximization already in the beginning of the next period then the relevant uncertainty measure 
would be the variance of $v_{k, t+1}$. Conceptually the expected length of the interval between two successive maximization dates (revision interval) resembles the length of delay period during which in the delayed-adjustment / inattentive consumer models consumers do not update their consumption (see e.g. Gabaix and Laibson, 2001; Reis, 2006). For expositional convenience, but without loss of qualitative generality, we assume in the following that the underlying data frequency and the consumption revision interval coincide. Now the statistical properties of the expected wealth based on differences in information content between two successive periods determine the relevant uncertainty measure of wealth ${ }^{3}$.

For that purpose, we define the period $t$ expected human wealth conditional for both the information available in the beginning of period $t$ and in the beginning of period $t+1$ :

$$
\begin{gathered}
E_{t} H_{k, t}=H_{k, t}^{E}=y_{t}+R(1-\pi) E_{t} H_{k, t+1} \\
E_{t+1} H_{k, t}=y_{t}+R(1-\pi) E_{t+1} H_{k, t+1}
\end{gathered}
$$

The difference of (10) and (9) determines the impact of the increment of period $t+1$ information on period t expected human wealth, if it were available already in the beginning of period $t$, i.e. ${ }^{4}$

$$
E_{t+1} H_{k, t}-H_{k, t}^{E}=R(1-\pi)\left[E_{t+1} H_{k, t+1}-E_{t} H_{k, t+1}\right]
$$

If the consumer has also period specific information, as we discussed in the previous section, then equation (7) implies that the square bracket term on the right hand side of (11) is equal to:

$$
\begin{aligned}
E_{t+1} H_{k, t+1}-E_{t} H_{k, t+1} & =\sum_{i=0}^{\infty}[R(1+\pi)]^{i} y_{k, t+1}+\sum_{j=1}^{i} \gamma^{j} y_{k, t+j} \varepsilon_{k, t+1+j} \\
-\sum_{i=0}^{\infty}[R(1+\pi)]^{i} y_{k, t}\left(1+\gamma \varepsilon_{k, t+1}\right)+\sum_{j=1}^{i} \gamma^{j} y_{k, t+j} \varepsilon_{k, t+1+j} & \\
& =\frac{1-\gamma}{1-\bar{R}(1-\pi)} \sum_{i=0}^{\infty}[R(1+\pi)]^{i} y_{k, t+i} \varepsilon_{k, t+1+i}
\end{aligned}
$$

Now, with the expected human wealth $H_{k, t}^{E}$ being determined by (7), equation (11) can be written in the form:

$$
E_{t+1} H_{k, t}=H_{k, t}^{E}\left(1+\frac{\bar{R}(1-\pi)(1-\gamma)}{1-\bar{R}(1-\pi) \gamma} \sum_{i=0}^{\infty} \alpha_{k, i} \varepsilon_{k, t+1+i}\right)
$$

where $\alpha_{k, i}=\frac{[R(1+\pi) \gamma]^{i} y_{k, t+i}}{\sum_{i=0}^{\infty}[R(1+\pi) \gamma]^{i} y_{k, t+i}}$ and, hence, $\sum_{i=0}^{\infty} \alpha_{k, i}=1$. We see that the more period specific information is available (the closer to unity $\gamma$ ), the smaller the addition of information and its impact on the expected human wealth. With $\gamma=1$, (13) coincides with perfect foresight.

Equations (13) define the expected human wealth including the related uncertainty in terms of one period addition in information content. The next step is to derive the deterministic wealth equivalent,

\footnotetext{
${ }^{3}$ It is quite straightforward to extend the analysis to account for the possibility that the time frequency in data is higher (e.g. one quarter) than the length of period (n quarters) that is relevant for determining the size of risk adjustment to human wealth, Willman (2007).

${ }^{4}$ We could also say that whilst $H_{t}^{E}$ is period t expectation, $E_{t+i} H_{t}$ is its period t+i realization and $H_{t}$ is its realization when $i \rightarrow \infty$.
} 
$\tilde{W}_{k, t}=V_{k, t-1} /(1-\pi)+\tilde{H}_{k, t}$, for which the consumer would be willing to exchange her wealth containing uncertain human wealth as defined by (13), i.e.

$$
E_{t+1} W_{k, t}=\underbrace{V_{k, t-1} /(1-\pi)+H_{k, t}^{E}}_{W_{k, t}^{E}}\left(1+\sum_{i=0}^{\infty} \phi_{k, i} \varepsilon_{k, t+1+i}\right)
$$

where $\phi_{k, i}=\frac{\bar{R}(1-\pi)(1-\gamma)}{1-\bar{R}(1-\pi) \gamma} \alpha_{k, i}$ with $0 \leq \sum_{i=0}^{\infty} \phi_{k, i} \leq R(1-\pi)$.

Utility equivalence, using the second order Taylor expansion, requires:

$$
\begin{gathered}
u\left(\tilde{W}_{k, t}\right)=E_{t}\left\{u\left(W_{k, t}^{E}+H_{k, t}^{E} \sum_{i}^{\infty} \phi_{k, i} \varepsilon_{k, t+1+i}\right)\right\} \\
\approx u\left\{W_{k, t}^{E}\right\}+\frac{1}{2} u "\left\{W_{k, t}^{E}\right\} E_{t}\left\{\left(H_{k, t}^{E} \sum_{i=0}^{\infty} \phi_{i} \varepsilon_{k, t+1+i}\right)^{2}\right\}
\end{gathered}
$$

where $u(\cdot)$ denotes the utility function and $u$ " $(\cdot)$ its second derivative. Assume the logarithmic utility function:

$$
u\left(\tilde{W}_{k, t}\right)=\log \tilde{W}_{k, t}
$$

Now equation (13) implies the following solution for the risk-adjusted human wealth ${ }^{5}$ :

$$
\tilde{H}_{k, t}=H_{k, t}^{E} \underbrace{\left[1-\left(\frac{H_{k, t}^{E}}{W_{k, t}^{E}}\right) \frac{\sigma_{t}^{2}}{2} \sum_{i=1}^{\infty} \phi_{i}^{2}\right]}_{\Lambda_{k, t}}
$$

Relation (16) shows that the risk adjusted human wealth can be presented as the multiplicand $\Lambda_{k, t}$ of the expected human wealth, which is a nonlinear function of the variance of stochastic labour income and the human wealth to total wealth ratio. Hence, we can present the risk-adjusted life-time budget constraint, which is relevant for the determination of the consumer's optimal (planned) consumption path, as follows:

$$
\sum_{i=0}^{\infty} R^{i}(1-\pi)^{i} c_{k, t+i}=\frac{V_{k, t-1}}{1-\pi}+\Lambda_{k, t} H_{k, t}^{E}=\tilde{W}_{k, t}
$$

\subsubsection{The life-time budget constraint with habit formation}

We next modify the life-time budget constraint to account for habit formation. The habit formation implies non-separability in utility over time. In internal-habit models, habit depends on a household's own past consumption and the household takes account of this when choosing how much to consume as e.g. in Muellbauer (1988), Muellbauer and Lattimore (1995), Sundaresan (1989) and Constantinides (1990). The simplest treatment of habit is to replace the argument $c_{k, t+i}$ in the utility function by $c_{k, t+i}^{*}=c_{k, t+i}-a c_{k, t+i-1}$, where the parameter $a$ measures habit persistence with $a>0$ and the term $a c_{k, t+i-1}$ is the time-varying habit level of consumption. Following Muellbauer (1988), after substituting $c_{k, t+i}^{*}+a c_{k, t+i-1}$ for $c_{k, t+i}$, the life-time resource constraint (17) can be modified to the form:

\footnotetext{
${ }^{5}$ In ending up the solution of $\tilde{H}$ corresponding the logarithmic utility function we applied the approximation $\log \left(\tilde{W} / W^{E}\right) \approx \frac{\tilde{H}-H^{E}}{W^{E}}$.
} 


$$
\sum_{i=0}^{\infty}[R(1-\pi)]^{i} c_{k, t+i}^{*}=(1-(1-\pi) a \bar{R}) \tilde{W}_{k, t}-a \cdot c_{k, t-1}
$$

\subsection{The optimal consumption rule and the aggregate consumption func- tion}

In this section we derive the optimal consumption rule for consumers in cohort $k$. Then we aggregate across cohorts and show that aggregate consumption can be expressed in terms of aggregate nonhuman and risk-adjusted human wealth.

Corresponding our earlier risk-analysis we assume that the consumer's preferences can be described by the logarithmic utility function. Hence, subject to the budget constraint (18), each consumer belonging to the cohort $k$ maximizes her expected inter-temporal utility:

$$
\max E_{t}\left[U_{k, t}\right]=E_{t}\left(\sum_{i=0}^{\infty}\left(\frac{1}{1+\rho}\right)^{i} u\left(c_{k, t+i}^{*}\right)\right)=\sum_{i=0}^{\infty}\left(\frac{1-\pi}{1+\rho}\right)^{i} \log \left(c_{k, t}^{*}\right)
$$

where parameter $\rho$ is the rate of subjective time preference. The resulting first-order condition of maximization is:

$$
c_{k, t+i}^{*}=c_{k, t}^{*} R^{-i}(1+\rho)^{-i}
$$

After substituting this condition to the risk-adjusted life-time budget constraint (18) we obtain:

$$
c_{k, t}=\kappa[1-(1-\pi) a \bar{R}]\left(\frac{V_{k, t-1}}{1-\pi}+\Lambda_{k, t} H_{k, t}^{E}\right)+a(1-\kappa) c_{k, t-1}
$$

where $\kappa=\frac{\rho+\pi}{1+\rho} \approx \rho+\pi$, and we see that via $\Lambda_{k, t}$ the consumption function (21) implies precautionary saving out of expected human wealth $H_{k, t}^{E}$.

Assume next that the size of each cohort when born is $\pi$. Accordingly, in period $t$ the size of cohort born in period $k$ is $\pi(1-\pi)^{t-k}$ and the size of population is $\sum_{k=-\infty}^{t} \pi(1-\pi)^{t-k}=1$. This results in the following aggregation rule: $z_{t}=\sum_{k=-\infty}^{t} \pi(1-\pi)^{t-k} z_{k, t}$ and $z_{t-1}=\sum_{k=-\infty}^{t-1} \pi(1-\pi)^{t-1-k} z_{k, t-1}$ with $z=\left\{c, H^{E}, \Lambda, V\right\}$. Following Blanchard (1985) we also assume that, except for across generation differences in stochastic income innovations, labour income is equally distributed across population. Now the aggregation of $(21)$ gives: $^{6}$

$$
c_{t}=\kappa[1-(1-\pi) a \bar{R}]\left(\frac{V_{t-1}}{1-\pi}+\Lambda_{t} H_{t}^{E}\right)+a(1-\kappa) c_{t-1}
$$

An interesting implication of aggregation is that, although over the life-cycle of an individual consumer, $\Lambda_{k, t}$, is time varying and strongly related to development of the expected human to total wealth ratio $\left(H_{k, t}^{E} / W_{k, t}^{E}\right)$, this does not necessarily imply that on the aggregate level $\Lambda_{t}$ should be non-stationary. In fact, under conventional assumptions of overlapping generation modelling, saving

\footnotetext{
${ }^{6}$ To end up with (22) the Taylor approximation is used: $\sum_{k=-\infty}^{t} \pi(1-\pi)^{t-k} \Lambda_{k, t} H_{k, t}^{E} \approx \Lambda_{t} H_{t}^{E}+$ $\sum_{k=-\infty}^{t} \pi(1-\pi)^{t-k} \Lambda_{t}\left(H_{k, t}^{E}-H_{t}^{E}\right)+\sum_{k=-\infty}^{t} \pi(1-\pi)^{t-k} H_{t}^{E}\left(\Lambda_{k, t}-\Lambda_{t}\right)=\Lambda_{t} H_{t}^{E}$
} 
and wealth accumulation across cohorts follow the same life-time profile (except for discrepancies resulting from stochastic income innovations) and, hence, the aggregate $\left(H_{t}^{E} / W_{t}^{E}\right)$ remains practically constant. Therefore, in the following our maintained hypothesis is that $\Lambda_{t}$ is stationary ${ }^{7}$.

To express (22) in terms of observable variables, we forward (22) by one period and take expectations. Thereafter we multiply it by $R_{t}(1-\pi) \gamma$, subtract it from (22) and utilize the aggregate-level dynamic budget constraint $V_{t}=R_{t}^{-1}\left[V_{t-1}+y_{t}-c_{t}\right]$ implied by (1) (compare to Blanchard, 1985; Gali, 1990). We end up with equation:

$$
\begin{array}{r}
\left\{1+\gamma\left[(1-\pi)(1-\kappa) a R_{t}-\kappa(1-(1-\pi) a \bar{R})\right]\right\} c_{t}=(1-\pi) \gamma R_{t} E_{t} c_{t+1}+a(1-\kappa) c_{t-1} \\
+(1-(1-\pi) \bar{R} a) \kappa\left\{\begin{array}{c}
\left(\frac{1}{1-\pi}-\gamma\right)\left(V_{t-1}+y_{t}\right) \\
+\left(\frac{\Lambda_{t}(1-(1-\pi) \bar{R} \gamma)}{1-(1-\pi) \bar{R}}-\frac{1}{1-\pi}\right) y_{t}
\end{array}\right\}
\end{array}
$$

Equations (23) covers a wide range of alternative cases. If $\pi=0$, the overlapping generations framework reduces to that of infinitely living representative agent framework. If no habit formation exists $(a=0)$, then consumption is determined by the beginning of period financial wealth and the future income stream. Consumption is the more forward-looking, the closer to unity the information parameter $\gamma$. In the opposite polar case with $\gamma=0$ consumers have no period-specific information and consumption is determined by the beginning of period financial wealth, current labour income and, with habit formation, the lagged consumption.

\section{Firm behaviour}

The firm maximizes its expected discounted stream of dividends, $V_{t}$. Without loss of generality, for notational simplicity, we assume that bank loans are the only form of external financing. We first define the determination of dividends in terms of income and cost components. Regarding cost, as in McAdam and Willman (2008), we allow a careful modelling of factor adjustment costs. Labour participation decisions are modeled along the intensive and extensive margins. In so doing, we introduce the concept of "effective labour hours". An innovative aspect is that the former margin turns out to have a key spillover onto firms' pricing decisions. Capital accumulation, in turn, reflects time-tobuild considerations. To capture price and inflation stickiness we adopt three valued Calvo-signalling mechanism, which as discussed in McAdam and Willman (2010), implies that each firm faces ex ante exactly the same optimization problem independently from the ex post outcome of the Calvo-signal. In addition to conventional demand and technology constraints the present value maximisation is constrained by non-negativity of gross investment and by the upper bound for the debt-to-capital stock ratio. Hence, the estimated aggregate level equations assume that for a certain percent of firms these constrains are binding. In addition, our aggregation accounts for the effects of structural changes in sectoral output shares from competitive (low mark-up) manufacturing to less-competitive (high-markup) services sectors. This phenomenon introduces secularly growing mark-up on aggregate price level as discussed by Willman (2002) and McAdam and Willman (2004).

\footnotetext{
${ }^{7}$ In general, however, it is possible that variation in the aggregate non-human to human wealth ratio affects the marginal propensity to consume out of expected human wealth. If true, this would introduce an additional nonlinearity into the wealth channel. However, besides being difficult to identify, the impact of this nonlinearity on aggregate consumption can be thought to be of second-order magnitude. This is very different from the role of the non-human to human wealth ratio in explaining cross-sectional differences in consumption, where this ratio can be thought to play the role of first order importance.
} 
We first present this framework using general functional notations and later, before deriving estimated specifications, present precise functional definitions of cost and production functions.

\subsection{Output, costs and dividends}

The output of a firm is defined by the production function,

$$
Y=F\left(K_{t}, H_{t}, t\right)
$$

$Y_{t}=$ output, $K_{t}=$ capital stock, $H_{t}=N_{t} h_{t}=$ labour input is measured in terms of 'effective' labour hours, $N_{t}=$ the number of employees, and $h_{t}=$ 'effective' working hours per employee.

From the point of view of production the relevant labour input concept is effective (or intensive) hours worked. Hence, a firm can increase labour input either by recruiting new employees or increasing the number of effective hours (via work intensity or overtime) per worker of existing employees. If wage agreements are basically fixed time contracts with overtime premium and, in addition, recruiting new employees and firing existing ones are associated with costs, then wage costs per efficient hour are above the contracted straight (normal) time rate both when effective hours $h_{t}$ are either below (as hours with low intensity are paid at the same rate as hours with normal intensity) or above normal hours $\bar{h}$. Hence, total wage costs can be presented as a convex function of the deviation of effective hours from normal hours.

$$
\bar{W}_{t} N_{t}\left[h_{t}+A_{h}\left(h_{t}-\bar{h}\right)\right] \quad ; A_{h}(0)=0 ; \frac{\partial A_{h}}{\partial h}\left\{\begin{array}{l}
>0 \text { if } h_{t}>\bar{h} \\
\leq 0 \text { if } h_{t} \leq \bar{h}
\end{array} \quad \text { and } \frac{\partial^{2} A_{h}}{\partial h^{2}} \geq 0\right.
$$

$A_{h}(\cdot)$ accounts for the cost effects of overtime wage premium and variations in work intensity. By assuming, as in Shapiro (1986) and Bils (1987), that all workers in the firm - and even more so across firms and industries - do not work the same number of hours nor at the same intensity, the function $A_{h}(\cdot)$ can be treated as continuously differentiable, although for an individual employee the wage rate may jump up discontinuously when his hired hours exceed normal hours $h-\bar{h}>0$.

Accounting also for adjustment costs associated with changes in both employment as well as with capital, real dividends $\left(\right.$ Div $\left._{t}\right)$ are determined as:

$$
\begin{aligned}
& \operatorname{Div}_{t}=Y_{t}-\frac{\bar{W}_{t}}{P_{t}}\left[N_{t} h+N_{t} A_{h}\left(h_{t}-\bar{h}\right)+A_{N}\left(N_{t}, N_{t-1}\right)\right] \\
& \quad-\left(K_{t}-(1-\delta) K_{t-1}\right)-A_{K}\left(K_{t}, K_{t-1}, K_{t-2}\right)+B_{t}^{j}-\left(1+r_{t-1}\right) B_{t-1}^{j}
\end{aligned}
$$

$P_{t}=$ output price; $\bar{W}_{t}=$ straight-time wage rate; $B_{t}=$ net real external debt financing and $r_{t}=$ the real interest rate.

Functions $A_{N}(\cdot)$ and $A_{K}(\cdot)$ represent adjustment cost functions for labour and capital, whose exact form, as the function of $A_{h}(\cdot)$, will be defined later. Note, that for capital, we assume that adjustment costs can additionally accommodate changes in the rate of capital stock accumulation; this extra cost essentially reflects time-to-build considerations in investment formation, and is empirically supported.

\subsection{Three-valued state dependent Arrow-Calvo signal}

At the beginning of each period, assume firms receive a time-varying signal regarding price setting in the following three-valued manner:

1./ With a probability $\theta_{t}$ firm $j$ receives the signal indicating that the firm is not allowed to change its price, i.e. $P_{t}^{j}=P_{t-1}^{j}$. As McAdam and Willman (2010) we allow the signal to be state dependent. i.e. its value depends on inflation and market structure. Specifically, we assume that in a 
high-inflation environment price changes are more frequent than otherwise. The following functional form captures these ideas:

$$
\theta_{t}=\theta \cdot\left(\frac{P_{t}}{P_{t-1}}\right)^{1-\varepsilon} \equiv \theta \cdot\left(1+\pi_{t}\right)^{(1-\varepsilon)}
$$

where $\theta \in[0,1]$ and $\theta_{\mathrm{t}} \in[0, \theta] \forall \pi_{t} \geq 0$; as we shall demonstrate, functional form (27) is particularly convenient to preclude linearization of the optimal reset price.

2./ With a probability $\left(1-\theta_{t}\right) \omega$ firm $\mathrm{j}$ is allowed to change its price following a backward-looking pricing rule, as in Gali and Gertler (1999), $P_{t}^{j}=P_{t}^{b}=\left(P_{t-1} / P_{t-2}\right) P_{t-1}^{v}$, where $P_{t-1}^{v}$ is the average price level selected by firms able to change price at time $\mathrm{t}-1$, and where $\omega \in[0,1]$ represents the fraction of firms able to reset prices but who do so in this rule-of-thumb manner.

3./ With a probability $\left(1-\theta_{t}\right)(1-\omega)$ firm $\mathrm{j}$ receives the signal that allows it to reset its price on the profit-maximization level, $P_{t}^{j}=P_{t}^{f}$.

Assume each firm solves its profit-maximization problem in the beginning of the period with full information on all current-period variables - except for the price-setting category to which it belongs ex-post. Regarding the reset signal itself, the prior probability distribution is known, i.e. $E_{t} \theta_{t}=\theta_{t}$ and, hence, the $j$-th firm's expected price level is the probability weighted average of possible outcomes,

$$
E_{t} P_{t}^{j}=\theta_{t} P_{t-1}^{j}+\left(1-\theta_{t}\right)\left\{(1-\omega) P_{t}^{f}+\omega P_{t-1}^{v}\right\}
$$

Although we find that at the firm level $E_{t} P_{t}^{j} \neq P_{t}^{j}$, the equality $E_{t} P_{t}=P_{t}$ continues to hold at the aggregate level. That is, because period $t$ aggregated price components (will be defined later) as well the weighting structure are known ex ante although individual firms do not know to which pricing category they belong ex post. Accordingly, in the context of optimizing the advantage of the three-valued signal is that in the beginning of each period, before the outcome of the signal is known, each firm faces exactly the same optimization problem, (i.e. in an ex-ante sense, all firms are profit-maximizers).

\subsection{The first order conditions of the profit maximising firm - dynamic Euler conditions}

Define a conventional isoelastic demand curve faced by firm $j$ as follows:

$$
Y_{t}^{j}=D\left(Y_{t}, P_{t}, P_{t}^{j}\right)=Y_{t}\left(\frac{P_{t}^{j}}{P_{t}}\right)^{-\varepsilon}
$$

where variables with superscripts refer to the firm $j$ and variables without superscript refer to the corresponding aggregate level variables. Real dividends of the firm $j$ are

$$
\begin{aligned}
D i v_{t}^{j} & =\frac{P_{t}^{j}}{P_{t}} D\left(Y_{t}, P_{t}, P_{t}^{j}\right)-\frac{\bar{W}_{t}}{P_{t}}\left[N_{t}^{j} h_{t}^{j}+N_{t}^{j} A_{h}\left(h_{t}^{j}-\bar{h}\right)+A_{N}\left(N_{t}^{j}, N_{t-1}^{j}\right)\right] \\
& -\left(K_{t}^{j}-(1-\delta) K_{t-1}^{j}\right)-A_{K}\left(K_{t}^{j}, K_{t-1}^{j}, K_{t-1}^{j}\right)+B_{t}^{j}-\left(1+r_{t-1}\right) B_{t-1}^{j}
\end{aligned}
$$

and the present value maximization problem of the firm $j$ is

$$
E_{t} \sum_{i=0}^{\infty} \beta_{t, t+i}\left\{\begin{array}{l}
D i v_{t+i}^{j}+\Lambda_{t+i}^{Y}\left[F\left(K_{t+i}^{j}, h_{t+i}^{j} N_{t+i}^{j}, t\right)-D\left(Y_{t+i}, P_{t+i}, P_{t+i}^{j}\right)\right] \\
+\Lambda_{t+i}^{B}\left(\alpha K_{t+i}^{j}-B_{t+i}^{j}\right)+\Lambda_{t+i}^{I}\left[K_{t+i}^{j}+(1-\delta) K_{t-1+i}^{j}\right]
\end{array}\right\}
$$


where $\beta_{t, t+i}$ refers to the discount rate and $\Lambda_{t+i}^{k}$ (with $k=Y, B, I$ ) refer to the Lagrangean multipliers associated with the demand constraint of output, the upper bound of the real debt-to-capital-stock ratio and the non-negativity of gross investment. After applying expectation rule (28) and observing that on the basis of $(27) \prod_{j=0}^{i} \theta_{t+j}=\theta^{i}\left(\frac{P_{t+i}}{P_{t}}\right)^{1-\varepsilon}$ the maximization problem (31) can be rewritten in the form ${ }^{8}$ :

$$
\operatorname{Max} \sum_{i=0}^{\infty} \beta_{t, t+i}\left\{\begin{array}{c}
\theta^{i}\left(1-\theta_{t}\right)(1-\omega)\left(\frac{P_{t}^{f}}{P_{t}}\right)^{1-\varepsilon} Y_{t+i} \\
-\frac{\bar{W}_{t+i}}{P_{t+i}}\left[N_{t+i}^{j} h_{+i t}^{j}+N_{t+i}^{j} A_{h}\left(h_{t+i}^{j}-\bar{h}\right)+A_{N}\left(N_{t+i}^{j}, N_{t-1+i}^{j}\right)\right] \\
-\left(K_{t+i}^{j}-(1-\delta) K_{t-1+i}^{j}\right)-A_{K}\left(K_{t+i}^{j}, K_{t-1+i}^{j}, K_{t-2+i}^{j}\right) \\
+B_{t+i}^{j}-\left(1+r_{t-1+i}\right) B_{t-1+i}^{j} \\
+\Lambda_{t+i}^{Y}\left\{F\left(K_{t}^{j}, H_{t}^{j}, t\right)-\theta^{i}\left(1-\theta_{t}\right)(1-\omega) P_{t}^{\varepsilon-1}\left(P_{t}^{f}\right)^{-\varepsilon} P_{t+i} Y_{t+i}\right\} \\
+\Lambda_{t+i}^{B}\left(\alpha K_{t+i}^{j}-B_{t+i}^{j}\right)+\Lambda_{t+i}^{I}\left(K_{t+i}^{j}-(1-\delta) K_{t-1+i}^{j}\right) \\
+\Lambda_{t+i}^{I}\left[K_{t+i}^{j}+(1-\delta) K_{t-1+i}^{j}\right]
\end{array}\right\}
$$

We maximize (32) with respect to $P_{t}^{f}, h_{t+i}^{j}, N_{t+i}^{j}, K_{t+i}^{j}$ and $B_{t+i}^{j}$. The first order condition of maximization with espect to $h_{t+i}^{j}$ implies that Lagrangean multiplier $\Lambda_{t+i}^{Y}$ can be expressed in terms of the real marginal cost of efficient hours as follows:

$$
\Lambda_{t+i}^{Y}=\frac{\bar{W}_{t+i}}{P_{t+i}} \frac{\left(1+\partial A_{h} / \partial h_{t+i}^{j}\right)}{\partial F / \partial H_{t+i}^{j}}=\frac{\bar{W}_{t+i}}{P_{t+i}} \frac{\left(1+\partial A_{h} / \partial h_{t+i}^{j}\right) h_{t+i}^{j}}{\partial F / \partial N_{t+i}^{j}}=M C R_{t+i}^{j}
$$

Likewise, the first order condition with respect to debt, $B_{t}^{j}$ results in:

$$
\beta_{t, t+1}=\frac{1-\Lambda_{t}^{B}}{1+r_{t}}=R_{t, t+1}\left(1-\Lambda_{t}^{B}\right)
$$

The first order conditions with respect to $N_{t}^{j}$ and $P_{t}^{f}$ together with (33) result in the following relations for (implicit) labour demand and optimal price setting:

$$
\begin{gathered}
\frac{\partial A_{N}\left(N_{t}^{j}, N_{t-1}^{j}\right)}{\partial N_{t}^{j}}-\frac{\partial A_{N}\left(N_{t+i}^{j}, N_{t}^{j}\right)}{\partial N_{t}^{j}}=\left(\frac{\partial A_{h}\left(h_{t}^{j}-\bar{h}\right)}{\partial h_{t}^{j}}-\frac{A_{h}\left(h_{t}^{j}-\bar{h}\right)}{h_{t}^{j}}\right) h_{t}^{j} \\
P_{t}^{f}=\frac{\varepsilon}{\varepsilon-1} \frac{\sum_{i=0}^{\infty} R_{t, t+i} \theta^{i} Y_{t+i} P_{t+i} M C R_{t+i}}{\sum_{i=0}^{\infty} R_{t, t+i} \theta^{i} Y_{t+i}}
\end{gathered}
$$

We have dropped the superscript $j$ in $M C R$, because all firms have same expectations and, hence, the same expected marginal $\operatorname{costs}^{9}$.

\footnotetext{
${ }^{8}$ For notational simplicity $(32)$ contains only the probability weighted demand steam conditional on $P_{t+i}^{j}=P_{t}^{f}$. All possible outcomes conditional on $P_{t+i}^{j} \neq P_{t}^{f}$ do not affect the solution of (31) and, therefore, they can be neglected in (32). We have also dropped expectation operator although all forward values of variables refer to their expectations rather than to their later actual realizations.

${ }^{9}$ Our implicit assumption is that $\Lambda_{t+i}^{B}=\Lambda_{t}^{B} \forall i \geq 0$.
} 
Relation (36) can be further simplified by assuming as e.g. Woodford (2003) that households have access to a complete set of contingent claims, and that identical consumers maximize their intertemporal utility, $\sum_{i} \beta^{i} U\left(C_{t+i}\right)$, we have for the discount rate $R_{t, t+i}$ :

$$
\frac{1}{R_{t, t+i}} \beta^{i} E_{t}\left[\frac{U_{c}\left(C_{t+i}\right)}{U_{c}\left(C_{t}\right)}\right]=1
$$

where $\mathrm{C}$ denotes consumption, and $\beta$ is the discount factor. For the logarithmic utility function and under the assumption that (expected) market growth equals (expected) consumption growth, $\frac{Y_{t+i}}{Y_{t}}=\frac{C_{t+i}}{C_{t}}$, equations (37)-(36) imply,

$$
P_{t}^{f}=\frac{\varepsilon}{\varepsilon-1} \frac{\sum_{i=0}^{\infty}(\beta \theta)^{i} P_{t+i} M C R_{t+i}}{\sum_{i=0}^{\infty}(\beta \theta)^{i}}=(1+\mu)(1-\beta \theta) \sum_{i=0}^{\infty}(\beta \theta)^{i} M C N_{t+i}
$$

where $\mu=(\varepsilon-1)^{-1}$ and $M C N$ are the mark-up and nominal marginal cost of labour, respectively. The logarithmic approximation of (38) can be written as,

$$
p_{t}^{f}=(1-\beta \theta) \sum_{i=0}^{\infty}(\beta \theta)^{i} E_{t}\left(m c n_{t+i}+\mu\right)
$$

Finally, the first order condition with respect to capital results in the relation:

$$
\begin{aligned}
& \frac{\partial A_{K}\left(K_{t}, K_{t-1}, K_{t-2}\right)}{\partial K_{t}}+ \beta_{t, t+1} \frac{\partial A\left(K_{t+1}, K_{t}, K_{t-1}\right)}{\partial K_{t}} \\
&+\beta_{t, t+2} \frac{\partial A\left(K_{t+2}, K_{t+1}, K_{t}\right)}{\partial K_{t}}=M C R_{t} \frac{\partial F\left(K_{t}, H_{t}, t\right)}{\partial K_{t}} \\
&-\left\{\left(1-\Lambda^{I}\right) U C_{t}+\Lambda^{B}(1-\delta)\left[\alpha-\left(1-\Lambda^{I}\right)\right]\right\}
\end{aligned}
$$

where

$$
U C_{t}=\frac{r_{t}+\delta}{1+r_{t}}
$$

After defining explicitly adjustment cost functions $A_{N}(\cdot)$ and $A_{K}(\cdot)$ as well as the cost function associated with the deviations of efficient hours from normal hours $A_{h}(\cdot)$, equations (35) and (40) determine dynamic demand for employees and capital and (39) determines the optimal price of firms, which are allowed to reset their prices optimally. An important driving variable, efficient hours $H_{t}$, is determined by the inverted production function (24). However, to be able to operationalise it as well as the marginal product of capital and labour needed by dynamic factor demand and price equations, we have to know the exact functional form and parameter values of the production function. Therefore we first specify the frictionless steady state implied by the dynamic first order conditions and the production function. As the Monte Carlo study by León-Ledesma, McAdam and Willman (2010) shows, this offers an efficient way to estimate the central parameters of the medium-run supply side. 


\subsection{Functional forms of the factor adjustment costs and the efficient to normal hours wage premium}

\subsubsection{Convex adjustment cost functions of labour and capital}

For estimation we must define explicit functional forms for adjustment cost functions $\mathrm{A}_{N}, \mathrm{~A}_{K}$. Where relations between variables are multiplicative, as here, quasi-quadratic adjustment costs result in particularly elegant results, Willman et al (2000). The adjustment cost functions, and for comparison, their quadratic counterparts are then,

$$
\begin{aligned}
A_{N}\left(N_{t}, N_{t-1}\right)= & \frac{a_{N}}{2} \cdot \Delta N_{t} \Delta n_{t} \approx \frac{a_{N}}{2} \cdot \frac{\left(\Delta N_{t}\right)^{2}}{N_{t-1}} \\
A\left(K_{t}, K_{t-1}, K_{t-2}\right)= & \frac{a_{K}}{2} \cdot \Delta K_{t} \Delta k_{t}+\frac{a_{K} b_{K}^{2}}{2} \cdot \Delta K_{t-1} \Delta k_{t-1} \\
& -a_{K} b_{K} \cdot \Delta K_{t} \Delta k_{t-1} \approx \frac{a_{K}}{2} \cdot \frac{\left(\Delta K_{t}-b_{K} \Delta K_{t-1}\right)^{2}}{K_{t-1}}
\end{aligned}
$$

where $n=\log (N)$ and $k=\log (K)$ and parameters $a_{N} \geqslant 0, a_{K} \geqslant 0, b_{k} \epsilon[0,1]$.

The advantage of the chosen functional form over its quadratic counterpart is that its differentials are log-linear and, across successive periods, symmetric. For instance

$$
\begin{gathered}
\frac{\partial\left(\frac{a_{N}}{2} \cdot \Delta N_{t} \Delta n_{t}\right)}{\partial N_{t}}=\frac{a_{N}}{2}\left(\Delta n_{t}+\frac{\Delta N_{t}}{N_{t}}\right) \cong a_{N} \Delta n \\
\frac{\partial\left(\frac{a_{N}}{2} \cdot \Delta N_{t+1} \Delta n_{t+1}\right)}{\partial N_{t}}=-\frac{a_{N}}{2}\left(\Delta n_{t+1}+\frac{\Delta N_{t+1}}{N_{t}}\right) \cong-a_{N} \Delta n_{t+1}
\end{gathered}
$$

\subsubsection{Efficient to normal hours wage premium}

In the spirit of indivisible labour (e.g., Kinoshita, 1987, Trejo, 1991, Rogerson, 1988) assume that contracts are drawn up in terms of fixed (or normal) working hours per employee, i.e. in terms of the straight-time wage rate. In general, effective hours in excess of normal hours attract a premium. Conversely, employers have limited possibilities to decrease paid hours when effective hours fall below normal ones. Hence, total wage costs can be presented as a convex function of the deviation of effective hours from normal hours. With normal hours normalised to unity, i.e $\bar{h}=1$, and using a variant of the "fixed-wage" model of Trejo (1991) for overtime pay, the following function gives a local approximation of this relation in the neighbourhood of effective hours equalling normal hours ${ }^{10}$ :

$$
W_{t}=\bar{W}_{t}\left[h_{t}+A_{h}\left(h_{t}-1\right)\right]=\bar{W}_{t}\left[h_{t}+\frac{a_{h}}{2}\left(h_{t}-1\right)^{2}\right] ; a_{h} \geq 0
$$

where $W_{t}$ is wage compensation per employee and $\bar{W}_{t}$ is the straight-time wage rate which each firm takes as given. Conditional on the contracted straight-time wage rate and the overtime wage premium function, effective hours are completely demand determined. Firms can also freely (but not costlessly) determine the allocation of total effective hours into effective hours per employee and the number of employees.

\footnotetext{
${ }^{10}$ Trejo's (1991) focus was in overtime hours and, therefore, he did not distinguish between effective and paid hours. Hence, our formulation is compatible with his when $h_{t} \geq \bar{h}$ and effective and paid hours are equal. However, our formulation also accounts for the possibility that effective hours are below normal (i.e. paid) hours.
} 


\subsection{Dynamic factor demand equations}

Substituting these cost functions (42)-(43) (and their derivatives) into (35) and (40) and utilizing the relation $W_{t}=\bar{W}_{t}\left[h_{t}+\frac{a_{h}}{2}\left(h_{t}-1\right)^{2}\right]$, we derive our final dynamic (estimable) factor demands,

\subsubsection{Labour demand}

$$
\Delta n_{t}=\frac{w_{t+1}}{\left(1+r_{t}+\kappa_{t}\right) w_{t}} \cdot \frac{\left(1+\log \left(\frac{F_{t}^{-1}}{N_{t}}\right)+a_{h}\left(\log \left(\frac{F_{t}^{-1}}{N_{t}}\right)\right)^{2}\right)}{\left(1+\log \left(\frac{F_{t+1}^{-1}}{N_{t+1}}\right)+a_{h}\left(\log \left(\frac{F_{t+1}^{-1}}{N_{t+1}}\right)\right)^{2}\right)} \cdot \Delta n_{t+1}+\frac{a_{h}}{a_{N}} \log \left(\frac{F_{t}^{-1}}{N_{t}}\right)
$$

where $w_{t}=W_{t} / P_{t}$ and the term $F^{-1}$ refers to the inverted production function solved for efficient hours $H_{t}$.

\subsubsection{Capital formation}

$$
\begin{aligned}
\frac{\left(1-\Lambda^{B}\right)^{2} b_{K}}{\left(1+r_{t}\right)\left(1+r_{t+1}\right)} \Delta k_{t+2} & -\left(\frac{\left(1-\Lambda^{B}\right)^{2} b_{K}^{2}}{\left(1+r_{t}\right)\left(1+r_{t+1}\right)}+\frac{\left(1-\Lambda^{B}\right)\left(1+b_{K}\right)}{\left(1+r_{t}\right)}\right) \Delta k_{t+1} \\
& +\left(\frac{\left(1-\Lambda^{B}\right) b_{K}\left(1+b_{K}\right)}{\left(1+r_{t}\right)}+1\right) \Delta k_{t}-b_{K} \Delta k_{t-1} \\
= & \frac{1}{a_{K}}\left(\frac{P_{t}}{\left(1+\mu_{t}\right) P_{t}^{I}} \frac{\partial F}{\partial K_{t}}-\left\{\left(1-\Lambda^{I}\right) U C_{t}+\Lambda^{B}(1-\delta)\left[\alpha-\left(1-\Lambda^{I}\right)\right]\right\}\right)
\end{aligned}
$$

In (48) $\partial F / \partial K$ refers to the marginal product of capital. In addition, in ending up relation (48) we allowed (unlike the simplified maximisation problem (32) assumes) the price of investment $P_{t}^{I}$, due to import content, to deviate from the output price and, therefore, we expressed real marginal cost $M C R_{t}$ in terms of investment rather than output price and then utilized the relation: $\frac{P_{t}}{P_{t}^{I}}=(1+\mu) \frac{M C N_{t}}{P_{t}^{I}}$.

\subsection{New Keynesian Phillips-curve for prices}

Three-valued Arrow-Calvo signal implies that the aggregate price, $p_{t}$, (lower case denoting logs) can be defined as the weighted sum of the reset and lagged price (see the detailed derivation in McAdam and Willman (2010))

$$
p_{t} \equiv\left(1-\theta_{t}\right) p_{t}^{v}+\theta_{t} p_{t-1}
$$

where

$$
p_{t}^{v}=\left(1-\omega_{p}\right) p_{t}^{f}+\omega p_{t}^{b}
$$

Inserting (50) into (49), subtracting $p_{t-1}$ from both sides and rearranging, yields,

$$
\left[\theta_{t}+\left(1-\theta_{t}\right) \omega_{p}\right] \Delta p_{t}=\left(1-\theta_{t}\right)\left[\left(1-\omega_{p}\right) p_{t}^{f}+\omega\left(p_{t-1}^{v}-p_{t-2}\right)\right]
$$

Furthermore, using (49) to solve for $p_{t-1}^{v}$ and inserting into (51), we derive,

$$
\left(\frac{\theta_{t}}{1-\theta_{t}}+\omega_{p}\right) \Delta p_{t}=\frac{\omega_{p}}{1-\theta_{t-1}} \Delta p_{t-1}+\left(1-\omega_{p}\right)\left(p_{t}^{f}-p_{t}\right)
$$


Next insert equation (39) into (52) to obtain:

$$
\left(\frac{\theta_{t}}{1-\theta_{t}}+\omega_{p}\right) \Delta p_{t}=\frac{\omega_{p}}{1-\theta_{t-1}} \Delta p_{t-1}+\left(1-\omega_{p}\right)\left(\left(1-\beta \theta_{p}\right)\left(\sum_{i=0}^{\infty}(\beta \theta)^{i} E_{t}\left(m c n_{t+i}+\mu\right)\right)-p_{t}\right)
$$

Expand the time-varying coefficients of (53) around the zero inflation rate:

$\left(\frac{\theta_{t}}{1-\theta_{t}}\right) \approx \frac{\theta_{p}}{1-\theta_{p}}+\left(\frac{\theta_{p}(1-\varepsilon)}{\left(1-\bar{\theta}_{p}\right)^{2}}\right) \Delta p_{t}$ and, $\left(\frac{\omega_{p}}{1-\theta_{t-1}}\right) \approx \frac{\omega_{p}}{1-\theta_{p}}+\frac{\omega_{p} \theta_{p}(1-\varepsilon)}{\left(1-\theta_{p}\right)^{2}} \Delta p_{t-1}$

After inserting these terms, equation (53) can be re-written as,

$$
\begin{aligned}
\left(\frac{\theta_{p}}{1-\theta_{p}}+\omega_{p}\right) \Delta p_{t}-\frac{\theta_{p}}{\left(1-\theta_{p}\right)^{2} \mu}\left(\Delta p_{t}\right)^{2}= & \\
& \frac{\omega_{p}}{1-\theta_{p}} \Delta p_{t-1}-\frac{\omega_{p} \theta_{p}}{\left(1-\theta_{p}\right)^{2} \mu}\left(\Delta p_{t-1}\right)^{2}+ \\
& \left(1-\omega_{p}\right)\left\{\left(1+\beta \theta_{p}\right)\left[\sum_{i=0}^{\infty}\left(\beta \theta_{p}\right)^{i} E_{t}\left(m c n_{t+i}+\mu\right)\right]-p_{t}\right\}
\end{aligned}
$$

and after substituting forward we derive

$$
\begin{aligned}
&\left\{\theta_{p}+\omega_{p}\left[1-\theta_{p}(1-\beta)\right]\right\} \Delta p_{t}-\omega_{p} \Delta p_{t-1}-\beta \theta_{p} E_{t} \Delta p_{t+1}= \\
&\left(1-\omega_{p}\right)\left(1-\theta_{p}\right)\left(1-\beta \theta_{p}\right)\left(m c n_{t}+\mu-p_{t}\right) \\
&+\frac{\theta_{p}}{\left(1-\theta_{p}\right) \mu}\left[\left(1-\beta \theta_{p} \omega_{p}\right)\left(\Delta p_{t}\right)^{2}-\omega_{p}\left(\Delta p_{t-1}\right)^{2}\right]
\end{aligned}
$$

This corresponds to a conventional hybrid NKPC as in Gali and Gertler (1999) except the last term containing quadratic inflation and the operational definition of the marginal cost variable. Regarding the quadratic inflation term that makes the (54) state dependent we, however, for simplicity neglect it in our estimation although, if desired, it can easily be introduced into relation, see McAdam and Willman (2010).

The first-order maximisation condition (33) defining the real marginal costs, the wage relation (46) defining compensation per employee, and the production function (24) solved for efficient hours $h_{t}=F^{-1}\left(K_{t}, Y_{t}, t\right) / N_{t}$ imply the following relation for nominal marginal costs:

$$
\begin{aligned}
\log M C N_{t} & =\log \left(\frac{\bar{W}_{t}}{\partial F / \partial N_{t}}\right)+\log \left(\frac{F^{-1}\left(K_{t}, Y_{t}, t\right)}{N_{t}}\right)+\log \left\{1+a_{h}\left[\left(\frac{F^{-1}\left(K_{t}, Y_{t}, t\right)}{N_{t}}\right)-1\right]\right\} \\
& \approx \log \left(\frac{W_{t}}{\partial F / \partial N_{t}}\right)+a_{h} \log \left(\frac{F^{-1}\left(K_{t}, Y_{t}, t\right)}{N_{t}}\right)
\end{aligned}
$$

\section{Union behaviour: Wage setting}

Consider an imperfectly competitive labour market, where a large number of monopoly unions determine real wages of their members under a right to manage structure (i.e. firms determine the employment level given the wage determined by unions). In renewing wage contracts each union sets 
the wage rate knowing its effects on employment determined by firms. Labour is indivisible so that variations in demand for labour determined by firms are transmitted to the number of unemployed instead of the hours worked per employee. In addition, assume that contracts are binding until they are renegotiated. That introduces stickiness in wage formation. In the following we first introduce Calvo staggering wage determination and thereafter we derive the determination of the optimal frictionless wage rate based on two alternative behavioural assumptions.

\subsection{Calvo staggering wage inflation}

To account for wage stickiness Calvo signaling mechanism in wage setting is assumed. Accordingly (i) with a probability $\theta_{w}$ union $j$ receives the signal indicating that the union is not allowed to change its price, i.e. $W_{t}^{j}=W_{t-1}^{j}$; (ii) with a probability $\left(1-\theta_{w}\right) \omega_{w}$ union $\mathrm{j}$ is allowed to change its price following a backward-looking rule, as in Gali and Gertler (1999) for price setting, $W_{t}^{j}=W_{t}^{b}=$ $\left(W_{t-1} / W_{t-2}\right) W_{t-1}^{v}$, where $W_{t-1}^{v}$ is the average price level selected by firms able to change price at time $t-1$, and where $\omega_{w} \in[0,1]$ represents the fraction of unions able to reset wages but who do so in this rule-of-thumb manner, which mimics the often observed catching up behaviour of many unions in response to earlier wage rises of other unions. Finally (iii) with a probability $\left(1-\theta_{w}\right)\left(1-\omega_{w}\right)$ union $j$ receives the signal that allows it to reset its wage rate on the desired optimal level, $W_{t}^{j}=W_{t}^{f}$.

As for prices, aggregation results in the following relation for wage inflation

$$
\left(\frac{\theta_{w}}{1-\theta_{w}}+\omega_{w}\right) \Delta w_{t}=\frac{\omega_{w}}{1-\theta_{w}} \Delta w_{t-1}+\left(1-\omega_{w}\right)\left(w_{t}^{f}-w_{t}\right)
$$

However, (56) is not operational, because the optimally reset wage level, $w_{t}^{f}=\log W_{t}^{f}$, is still undefined. For defining $w_{t}^{f}$ we follow Rotenberg (1987) and describe unions optimization process as a two-step procedure. In the first step, optimising unions determines the optimal wage rate $w_{t}^{*}$ and its expected path in the absence of all frictional elements in wage setting. Then in the second step forward-looking unions set the wage rate $w_{t}^{f}$ so that it minimizes a quadratic loss function that depends on the difference between the reset price over the periods it is expected to remain fixed and the optimal wage level maximizing the utility of representative member in the absence of restriction in wage setting. Hence, the optimizing unions minimize the function $\frac{1}{2} \sum_{i=0}^{\infty}\left(\beta \theta^{w}\right)^{i} E_{t}\left(w_{t}^{f}-w_{t}^{*}\right)^{2}$, which implies the following optimal reset wage rate:

$$
w_{t}^{f}=(1-\beta \theta) \sum_{i=0}^{\infty}(\beta \theta)^{i} E_{t} w_{t+i}^{*}
$$

After inserting (57) into (56) we obtain

$$
\left(\frac{\theta_{w}}{1-\theta_{w}}+\omega_{w}\right) \Delta w_{t}=\frac{\omega^{w}}{1-\theta^{w}} \Delta w_{t-1}+\left(1-\omega_{w}\right)\left\{\left(1+\beta \theta_{w}\right)\left[\sum_{i=0}^{\infty}\left(\beta \theta_{w}\right)^{i} E_{t} w_{t+i}^{*}\right]-w_{t}\right\}
$$

After substituting forward (58) can written in the conventional NKPC form:

$$
\left\{\theta_{w}+\omega_{w}\left[1-\theta_{w}(1-\beta)\right]\right\} \Delta w_{t}=\omega^{w} \Delta w_{t-1}+\beta \theta^{w} E_{t} \Delta w_{t+1}+\left(1-\omega_{w}\right)\left(1-\theta_{w}\right)\left(1-\beta \theta_{w}\right)\left(w_{t}^{*}-w_{t}\right)
$$

We next present the determination of the frictionless optimal wage rate $\mathrm{w}_{t}^{*}$ based on two alternative behavioural assumptions. 


\subsection{The frictionless wage rate}

We assume that part of the unions are utilitarian, maximising the utility of member households, whilst the rest are non-utilitarian keeping wage development in line with productivity development coupled with a high desired employment rate.

\subsubsection{The wage setting of utilitarian unions}

Assume as Hansen (1985) that through union membership employees have access to an insurance market which allows them to insure their income with respect of being unemployed. If employed, the employee receives compensation equalling $W * \bar{h}$, where $W$ contract wage and $\bar{h}$ is fixed hours worked. If unemployed, the employee gets unemployment compensation B. However, for the unemployment insurance union members have to pay membership fee covering paid insurance benefits. By assuming the logarithmic utility function the maximization problem of an utilitarian monopoly union under a right to manage structure is

$$
\begin{aligned}
& \operatorname{Max} L=n_{t}\left(\log C_{t}^{E}-\kappa\right)+\left(1-n_{t}\right) \log C_{t}^{U}+\lambda^{E} n_{t}\left[a_{t}+\left(W_{t} / P_{t}^{C}\right) \bar{h}-\left(1-n_{t}\right) B_{t} / P_{t}^{C}-C_{t}^{E}\right] \\
& +\lambda^{U}\left(1-n_{t}\right)\left[a_{t}+B_{t} / P_{t}^{C}-\left(1-n_{t}\right) B_{t} / P_{t}^{C}\right]+\lambda^{F}\left[F_{N}-(1+\mu) W_{t} / P_{t}\right]
\end{aligned}
$$

where $C_{t}^{E}$ and $C_{t}^{U}$ are consumption of employed and unemployed union member, $\mathrm{n}_{t}$ is the the probability to be employed (i.e. the employment rate $N_{t} / N_{t}^{F}$ with $N_{t}$ referring to the total number of employees and $N_{t}^{F}$ to the labour force). $\lambda^{E}$ and $\lambda^{U}$ are Lagrangian multipliers related to the budget constraint of employed and unemployed union members, respectively, and $\lambda^{F}$ the Lagrangian multiplier related to the labour demand of the profit maximizing firm. $a_{t}$ is the initial real wealth of (identical) consumers, $F_{N}$ is the marginal product of labour, $P_{t}$ is the deflator of production and $P_{t}^{C}$ is the deflator of consumption.

It is straightforward to show that the maximization of (60) with respect to $C_{t}^{E}, C_{t}^{U}$ and $B_{t}$ implies $\lambda^{E}=\lambda^{U}=1 / C_{t}^{E}=1 / C_{t}^{U}$ and $B_{t}=W * \bar{h}$ and the union maximization problem (60) can be reduced into the form

$$
M a x L=\log C_{t}-n_{t} \kappa+\lambda^{C}\left(a_{t}+n_{t} \bar{h} W_{t} / P_{t}^{C}-C_{t}\right)+\lambda^{F}\left[F_{N}\left(n_{t} N^{F}, K_{t}\right)-(1+\mu) W_{t} / P_{t}\right]
$$

The maximisation of $(61)$ with respect to consumption $\mathrm{C}_{t}$, the employment rate $\mathrm{n}_{t}$ and real wage rate $W_{t} / P_{t}^{C}$ gives, after some manipulation, the following relation for the real wage

$$
\frac{W_{t}}{P_{t}^{C}}=\frac{\kappa F_{N} C_{t}}{\bar{h}\left(F_{N}+N_{t} \frac{\partial F_{N}}{\partial N_{t}}\right)}
$$

In the case of the normalised CES production function (see Section 7):

$\frac{\partial F_{N}}{\partial N}=\frac{1}{\sigma} \frac{F_{N}^{C E S}}{N}\left(\frac{F_{N}^{C E S}}{Y / N}-1\right)$, where $F_{N}^{C E S}=\left(1-\pi_{0}\right)\left(\frac{Y_{0}}{N_{0}} \Gamma_{N}\left(t, t_{0}\right)\right)^{\frac{\sigma-1}{\sigma}}\left(\frac{Y_{t}}{N_{t}}\right)^{\frac{1}{\sigma}} ; \sigma>0$ is the elasticity of substitution between capital and labour and $\Gamma_{N}$ is labour augmenting technical progress. Recalling that $C_{t}$ is the consumption of an individual union member, which can be expressed in terms of aggregate data as $C_{t}=\tilde{C}_{t} / N_{t}^{F}$ (where $\tilde{C}_{t}$ is aggregate private consumption). Now (62) implies the following relation for the wage rate

$$
w_{t}=\left(p_{t}^{C}+\tilde{c}_{t}-n_{t}^{F}\right)-\log \left(\sigma-1+\frac{F_{N}^{C E S}}{Y_{t} / N_{t}}\right)+\log \frac{\sigma \kappa}{\bar{h}}
$$

Lower case letters refer to the logs of the corresponding upper case variables. 


\subsubsection{The wage setting of non-utilitarian unions}

Unions know that the frictionless real wage rate is defined by the profit maximising condition of the firm $W_{t} / P_{t}=F_{N}\left(N_{t}, K_{t}\right) /(1+\mu)$. In equilibrium this condition holds for all possible levels of employment and unions know that by fixing the nominal wage rate they also fix the (equilibrium) employment rate. By assuming that the aim of non-utilitarian unions is to keep the employment rate on some desired level $\varpi$, then we get the following relation for the warranted wage rate that fulfils this aim:

$$
W_{t}=\left(\frac{h_{t} N_{t}}{\varpi \cdot \bar{h} \cdot N_{t}^{F}}\right)^{\chi} \frac{P_{t} F_{N}}{1+\mu} ; 0<\varpi \leq 1, \chi>0
$$

If all unions are non-utilitarian, then in the full model context the relation (64) implies a constant long-run natural rate of unemployment. If instead, all or part of unions are utilitarian, then shocks that affect the demand structure, e.g. a permanent government expenditure shock affecting the GDP share of private consumption, affect also the long-run equilibrium unemployment rate.

\subsubsection{The frictionless optimal wage rate}

For estimating the NKPC wage equation (59), we define the frictionless wage rate $\mathrm{w}_{t}^{*}$ as a weighted average of the rates implied by (63) and (64). Hence, we write:

$$
w_{t}^{*}=a_{w u} \cdot w u_{t}^{*}+\left(1-a_{w u}\right) \cdot w n u_{t}^{*}
$$

where

$$
\begin{gathered}
w u_{t}^{*}=\left(p_{t}^{C}+\tilde{c}_{t}-n_{t}^{F}\right)-\log \left(\sigma-1+\frac{F_{N}^{C E S}}{Y_{t} / N_{t}}\right)+\log \frac{\sigma \kappa}{\bar{h}} \\
w n u_{t}^{*}=p_{t}+\log \left(\frac{F_{N}}{1+\mu}\right)+\chi \log \left(\frac{F^{-1}\left(K_{t}, Y_{t}\right)}{\varpi \cdot N_{t}^{F}}\right)
\end{gathered}
$$

\section{The Foreign trade block}

\subsection{Exports and export prices}

The first-order conditions of an optimising firm that produces export goods, through the combination of labour, capital and imported goods, allow us to write export prices as a mark-up over marginal costs. We assume that the demand for exports takes an almost ideal demand system (AIDS) form, depending on world demand for exports, Denton and Muellbauer (1980). The advantage of this representation is that the elasticity of the demand is no longer constant but depends on the relative competitor export prices. Profit maximisation under the almost ideal demand system type of export demand function results in the following 2-equation system for the export volume and export price, Willman and Estrada (2002):

$$
\left(\frac{P_{X} X}{P_{c x} M F}\right)=a+b \cdot f(\text { time })-(\phi-1)\left(p_{X}-p_{C X}\right)
$$




$$
\begin{aligned}
p_{X}=a+\frac{1+(a+b \cdot f(\text { time })) /(\phi-1)}{2+(a+b \cdot f(\text { time })) /(\phi-1)}\left(\left(1-a_{x}\right)(w-\right. & \text { mpn } \left.)+a_{x} p_{M}\right) \\
& +\frac{1}{2+(a+b \cdot f(\text { time })) /(\phi-1)} p_{C X}
\end{aligned}
$$

$P_{X}=$ Export deflator; $X=$ Export volume; $P_{C X}=$ the external competitor export prices; $M F=$ the world demand for exports; $w=$ compensation per worker; $m p n=$ marginal product of labour; $p_{M}=$ import deflator; $a=$ point market share (with indexed data close to unity); $\phi>1$ is the representative point price elasticity of exports; $b$ if different from zero measures the deviation of income elasticity of export demand from unity; and $a_{X}=$ import content of exports (input-output estimate). Lower case letters refer to the logs of variables. In estimating the system (66)-(67) an additional trend variable was allowed in the price equation.

\subsection{Imports and import prices}

The modelling approach of imports is conventional. The import supply curve is assumed to be horizontal and, hence, import volume is demand determined. Accordingly two driving variables are the domestic demand indicator WER and the relative price of domestic production and imports. Domestic indicator is determined as a weighted sum of domestic and export demand components. Single year estimates of the import content of each demand component are used as weights. However, accounting for the fact that the growth of world trade has been faster than the growth of world GDP that on a single county level can be seen as growing GDP shares of both imports and exports we add to the import equation the ratio of exports to the constructed demand indicator. As world trade increasingly is the trade of intermediaries this should especially be seen as the rising import share of exports. Hence, we specify the long-run equilibrium aggregate demand for imports $M$ to depend on the demand indicator (with unit elasticity), the ratio of exports to the demand indicator and the relative domestic-to-import price:

$$
m=\alpha_{1}+\alpha_{2}\left(p-p_{M N}\right)+w e r+\alpha_{3}(x-w e r)
$$

The import price $\mathrm{p}_{M N}$ excludes the imports of energy and lower-case letters refer to the logs of variables.

For forecasting purposes the import deflator is divided into energy and non-energy component. The price elasticity of imported energy is assumed to zero that is a reasonable approximation in the forecasting horizon although less realistic from the long-run perspective. The equation for the import deflator excluding energy is quite traditional. It depends on the GDP deflator (to capture possible pricing to market effects) net of indirect taxes, and the competitors' import price with static homogeneity condition imposed

$$
p_{M N}=\phi_{1}\left(p+\log \left(1-T X_{1}\right)\right)+\left(1-\phi_{1}\right) p_{C M}
$$

The GDP deflator at factor cost is included in the specification in order to capture the pricingto-market behaviour of the foreign firms. Therefore, this specification implies that energy prices have some second round effects on non-energy import prices. Equation (69) defines the equilibrium relation, while the dynamic specifications are conventional error-correction equations. The aggregate import deflator is determined as a weighted average of imported energy price $P E$ and the non-energy import price $P M N$ (in logs) :

$$
p_{M}=\alpha_{E} p_{E}+\left(1-\alpha_{E}\right) p_{M N}
$$




\section{Production function and the medium-run supply system}

In this section we start by specifying the explicit form of the production function. Thereafter we present the 5-equation medium-run supply-system implied by the first order conditions of profit maximization of the firm and the utility maximisation of labour unions. The parameter estimates of this system defines empirically the production function, the marginal products of inputs and the mark-up needed in estimating dynamic labour demand, capital formation, inventory, export, price and wage equations.

\subsection{The Normalised CES production function}

Our technology assumption is the "normalized" CES function allowing for time-varying factor augmenting technical progress. The importance of explicitly normalizing CES functions was discovered by La Grandville (1989) and first implemented empirically by Klump, McAdam and Willman (2007). Normalization starts from the observation that a family of CES functions whose members are distinguished only by different substitution elasticities need a common benchmark point. Since the elasticity of substitution is defined as a point elasticity, one needs to fix benchmark values for the level of production, factor inputs and marginal rate of substitution, or equivalently for per-capita production, capital deepening and factor income shares. The normalized CES production function corresponding to the general function $(24)$ is given $b^{11}$ :

$$
\frac{Y_{t}}{Y_{0}}=\left\{\pi_{0}\left[\Gamma_{K}\left(t, t_{0}\right) \frac{K_{t}}{K_{0}}\right]^{\frac{\sigma-1}{\sigma}}+\left(1-\pi_{0}\right)\left[\Gamma_{N}\left(t, t_{0}\right) \frac{h_{t} N_{t}}{h_{0} N_{0}}\right]^{\frac{\sigma-1}{\sigma}}\right\}^{\frac{\sigma}{\sigma-1}}
$$

where $\sigma$ is the elasticity of substitution between capital and labour, $\pi_{0}$ distribution parameter equalling the capital share evaluated at the normalization point (subscript 0$)$ and $\Gamma_{i}\left(t, t_{0}\right)$ define the (indexed) level of technical progress associated to factor $i$ (with $\Gamma_{i}\left(t_{0}, t_{0}\right)=1$ ).

\subsubsection{Technical progress}

As it is not obvious that growth rates should always be constant, we follow an agnostic approach and model technical progress drawing on a well-known flexible, functional form (Box and Cox, 1964):

$$
\log \left[\Gamma_{i}\left(t, t_{0}, \gamma_{i}, \lambda_{i}\right)\right]=\frac{\gamma_{i} t_{0}}{\lambda_{i}}\left[\left(\frac{t}{t_{0}}\right)^{\lambda_{i}}-1\right]
$$

where $i=N, K$. The $\log$ level of technical progress, $\Gamma_{i}(\bullet)$ is, therefore, a function of time, $\mathrm{t}$ (around its normalization point $t_{0}$ ), a curvature parameter, $\lambda_{i}$, and has a growth rate of $\gamma_{i}$ at the representative point of normalization. ${ }^{12}$ When $\lambda_{i}=1(=0)[<0]$, technical progress displays linear (log-linear) [hyperbolic] dynamics:

$$
\log \Gamma_{\mathrm{i}}(t) \Rightarrow \begin{cases}\lim _{t \rightarrow \infty}\left[\log \Gamma_{i}(\mathrm{t})\right]=\infty & \text { if } \lambda_{i} \geqslant 0 \\ \lim _{t \rightarrow \infty}\left[\log \Gamma_{i}(\mathrm{t})\right]=-\frac{\gamma_{i} t_{0}}{\lambda_{i}}>0 & \text { if } \lambda_{i}<0\end{cases}
$$

\footnotetext{
${ }^{11}$ León-Ledesma, McAdam and Willman (2009) discuss and evaluate normalization more extensively.

${ }^{12}$ Note we scaled the Box-Cox specification by to to interpret $\gamma_{N}$ and $\gamma_{K}$ as the rates of labour- and capitalaugmenting technical change at the fixed (i.e., representative) point.
} 


$$
\begin{aligned}
& \frac{\partial \log \Gamma_{i}(\mathrm{t})}{\partial \mathrm{t}}=\gamma_{i}\left(t / t_{0}\right)^{\lambda_{i}-1}
\end{aligned}
$$

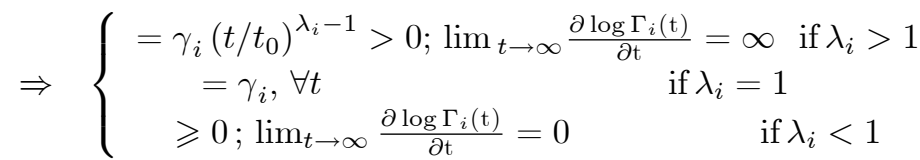

Thus, if $\lambda_{i} \geq 0$, the level of technical progress accruing from factor i tends to infinity but is bounded otherwise (73). If $\lambda_{i}=1$ the factor growth of technical progress is constant (i.e., the "text-book" case) but asymptotes to zero from above for any $\lambda_{i}<1,(74)$.

\subsection{The supply system}

The steady-state form of the three optimising private sector decision making units, i.e. firms, trade unions and households can be reduced to a four-equation supply-side system, which allows an efficient way to estimate the parameters of the production function, León-Ledesma et al. (2009). It is straight forward to see that in a frictionless world with $A_{N}(\cdot)$ and $A_{K}(\cdot)$ vanishing efficient hours $h_{t}$ equals $\bar{h}$, which is convenient to normalize to unity. Also the straight-time wage rate $\bar{W}_{t}$ equals wage compensation per employee $W_{t}$ and, hence, real marginal cost defined by (33) is $M C R_{t}=\frac{W_{t}}{P_{t}} / \frac{\partial F}{\partial N_{t}}$. Dynamic Euler equation (35) disappears as its both sides equal zero and conditions (38)-(40) reduce to the first order conditions of maximisation with respect to labour and capital of the conventional static optimisation framework. In addition, Willman (2002) and McAdam and Willman (2004) showed that, if income and price elasticities of demand deviate across production sectors, then the aggregation of the first order conditions of maximization and the production function introduces a trend into the aggregate data mark-up although all firm level (or sectoral) mark-ups would remain constant. Further, by incorporating into the system optimal (frictionless) wage setting equation (64) the steady-state system can be transformed into a full-fledged five-equation supply system Accounting for this effect, the static aggregated system of first order conditions and the production function supplemented with the implied mark-up equation can be written as follows

$$
\begin{gathered}
\log \left(\frac{P_{t}^{y} Y_{t}}{w_{t} N_{t}+q_{t} K_{t}}\right)-\log (1+\mu(t))=0 \\
\log \left(\frac{w_{t} N_{t}}{P_{t}^{y} Y_{t}}\right)-\log (1-\bar{\pi})-\frac{1-\sigma}{\sigma}\left[\log \left(\frac{Y_{t} / \bar{Y}}{N_{t} / \bar{N}}\right)-\log \xi-\frac{\bar{t} \gamma_{N}}{\lambda_{N}}\left(\left(\frac{t}{\bar{t}}\right)^{\lambda_{N}}-1\right)\right] \\
+\log (1+\mu(t))=0 \\
\log \left(\frac{q_{t} K_{t}}{P_{t}^{y} Y_{t}}\right)-\log (\bar{\pi})-\frac{1-\sigma}{\sigma}\left[\log \left(\frac{Y_{t} / \bar{Y}}{K_{t} / \bar{K}}\right)-\log \xi-\frac{\bar{t} \gamma_{K}}{\lambda_{K}}\left(\left(\frac{t}{\bar{t}}\right)^{\lambda_{K}}-1\right)\right] \\
+\log (1+\mu(t))=0
\end{gathered}
$$




$$
\begin{aligned}
& \log \left(\frac{Y_{t} / \bar{Y}}{N_{t} / \bar{N}}\right)-\log (\xi)-\frac{\bar{t} \gamma_{N}}{\lambda_{N}}\left(\left(\frac{t}{\bar{t}}\right)^{\lambda_{N}}-1\right)+ \\
& \frac{\sigma}{1-\sigma} \log \left[\bar{\pi} e^{\frac{1-\sigma}{\sigma}\left[\frac{\bar{y}_{N}}{\lambda_{N}}\left(\left(\frac{t}{t}\right)^{\lambda_{N}}-1\right)-\frac{\bar{t} \gamma_{K}}{\lambda_{K}}\left(\left(\frac{t}{t}\right)^{\lambda_{K}}-1\right)\right]}\left(\frac{K_{t} / \bar{K}}{N_{t} / \bar{N}}\right)^{\frac{\sigma-1}{\sigma}}+(1-\bar{\pi})\right]=0 \\
& \log \left(\frac{N_{t}^{F} w_{t}}{P_{t}^{c} C_{t}}\right)+\log \left\{\sigma-1+(1-\bar{\pi})\left[\frac{Y_{t} /(\xi \cdot \bar{Y})}{\frac{N_{t}}{N} e^{\frac{t_{N}}{\lambda_{N}}\left(\left(\frac{t}{t}\right)^{\lambda}-1\right)}}\right]^{\frac{\sigma-1}{\sigma}}\right\}-\log \left(\frac{\sigma \kappa}{\bar{h}_{t}}\right)=0
\end{aligned}
$$

where $Y, N$ and $K$ refer to output, employment and capital. $P_{y}, w$ and $q$ are their respective prices; $C, \quad P_{c}$ and $N^{F}$ are consumption, consumption deflator and labour force. Bars above the variables refer to the sample averages. ${ }^{13}$ Normalised production function implies that $\vec{\pi}=\frac{\bar{q} \bar{K}}{\bar{w} N+\bar{q} \bar{K}}$ is the capital share evaluated at the fixed point (sample mean).

Aggregation across heterogeneous sectors facing differentiated price and income elasticities of demand introduces a trend component into the mark-up as shown in (76). The task of this equation is to control this common trend component in equations (77) and (78) which are the steady state forms of the first order conditions of profit maximisation with respect of labour and capital. Equation (79) is the production function (71) after taking logs on its both sides. Equation (80) determining the optimal frictionless wage rate is the first order condition of maximisation of the utilitarian trade union under the right-to-manage structure, as is shown in section 4. It is part of the supply side system as it is conditional to the same production technology as the firm's maximisation conditions. In estimating of (80) variable $\bar{h}_{t}$, referring to average normal working hours per employee, was also allowed to include a box-cox trend to account for observed decrease in total hours per employee ratio.

\section{Estimation of the model}

The country models have been designed from the beginning to have a common underlying structure, but a structure that is general enough to allow for cross-country differences. In particular the CES structure with non-unit elasticity of substitution and varying technical progress gives flexibility in the production process. Furthermore the other equations reflect cross-country differences due to differences in the estimated parameters, e.g. the estimated New Keynesian Phillips curves contain both forward and backward looking behaviour, the relative weights of which vary across countries. Indeed, the modelling approach has been, within the constraints of the common theoretic setting, to allow the data to determine estimated parameter values, with only a limited amount of constraints.

\subsection{Data for estimation}

The statistical data used to estimate the model is based on quarterly data from 1980 to 2007 Q2. The data follow the new European System of National Accounts (ESA95) methodology, therefore the main source for the country data is Eurostat and in particular the European quarterly national accounts, from where information concerning GDP and main expenditure components, income, employment and compensation is taken. The ESA95 version was considered, making also use of accounts based

\footnotetext{
${ }^{13}$ We have defined the point of normalisation so that $t_{0}=\bar{t}, N_{0}=\bar{N} . K_{0}=\bar{K}, Y_{0}=\xi \bar{Y}$ and $\pi_{0}=\bar{\pi}$. Parameter $\xi$ is a normalization constant (close to unity) that resulting from nonlinearities the sample average of production need not exactly coincide with the level of production implied by the sample averages of inputs and time, Klump et al. (2007).
} 
on SNA79 in order to back-date variables. Eurostat is also the source for the Harmonised Index of Consumer Price Indexes and sub-components. In this case the back-dating of variables was based on Consumer Price Indexes.

As concerns financial variables, 3-month EURIBOR was used as the short-term interest rate measure and the 10-year bond yields as the long-term one. For stock prices the most representative indexes are considered (i.e. DAX for Germany, Ibex for Spain, Amsterdam AEX for Netherlands and MIB for Italy). Other variables like house prices or capital stock are based on internal calculations undertaken within the Eurosystem. The construction of world demand and competitors exports and import price variables is described in Hubrich and Karlsson (2010). Moreover, time series for a large number of model variables have been created applying model identities/definitions.

\subsection{Estimation of the supply system}

The steady state form of the first-order conditions of the profit maximising firm and unions maximising the utility of member households imply the 5-equation medium-run supply system (76)-(80) that allows a consistent two-step estimation of the underlying deep parameters of the model. As the supply system contains cross-equation parameter constraints, it is estimated with the method of non-linear SUR, see León-Ledesma et al. (2009), and which has proven to be a very efficient estimation approach outperforming all single-equation methods.

In estimating the system (76)-(80) we also studied whether, instead of developing smoothly over the sample period, there were breaks in the speed of factor augmenting technical progress to capture the effect of the IT-boom. We found that in all countries, except in Germany and the Netherlands, there were shifts in technical progress from the labour augmenting towards the capital augmenting progress around the years 1996-1997. Likewise to improve the data compatibility of the system we also allowed breaks in the trends of mark-up and normal working hours per employee.

According to our estimation results, capital augmentation was the dominating form of technical progress in all five countries, especially in the latter part of our estimation period, indeed, both the German and Dutch data favoured solely capital augmenting technical progress over the whole estimation period. Also in line with observed deceleration of labour productivity growth the curvature parameter estimates of $\lambda_{i}$ were below unity indicating gradually decelerating growth contributions of technical progress. The estimates of the key technology parameter, the elasticity of substitution between capital and labour are shown below. They are uniformly in all countries close to 0.6 implying a stark deviation from the unit elasticity of the Cobb Douglas production function. This has important implications to of the transmission mechanism of the whole model, where the elasticity of substitution is one of the key parameters.

Production Function Estimates
\begin{tabular}{|l|l|l|l|l|l|}
\hline & FR & DE & IT & ES & NL \\
\hline Elasticity of & 0.532 & 0.614 & 0.614 & 0.550 & 0.575 \\
Substitution* & $(0.0005)$ & $(0.0006)$ & $(0.0006)$ & $(0.0036)$ & $(0.0080)$ \\
\hline $\begin{array}{l}\text { Long-run Esti- } \\
\text { mation sample }\end{array}$ & $81 \mathrm{q} 2$ & $93 \mathrm{q} 1$ & $87 \mathrm{q} 1$ & $83 \mathrm{q} 1$ & $81 \mathrm{q} 1$ \\
\hline
\end{tabular}

* Standard errors of estimates in brackets.

This system defines the parameters related to technology, production function and the mark-up allowing to define optimal frictionless prices, wages, labour demand and marginal cost and product concepts needed in estimating in the second stage the dynamic first order optimisation conditions of firms and unions. 


\subsection{Dynamic Equation Estimation}

All dynamic equations containing the leads of variables are estimated by the generalised method of moment (GMM), Hansen (1982). GMM is a limited information method that can be applied without a full statistical specification of all variables of the model as should be the case in applying some variant of the full information maximum likelihood method (FIML). It is, however, able to solve the problem of serially correlated errors resulting from the replacement of expectations by actual realisations in estimated specifications. Therefore, in the present context, where optimizing agents are assumed to be boundedly rational in knowing only the parameters related to their problem but not necessarily the rest of the model, GMM is an especially appealing estimation method. As a partial, single equation method, GMM is not quite as efficient as FIML but, as opposed to FIML estimates, GMM estimates are not contaminated by possible misspecifications in the rest of the model.

Hence, GMM is an estimation technique based on minimal assumptions which does not require us to specify an explicit process for the underlying driving variables instead an instrument set is used for the expected variables without referring to the structure of the process driving the forcing variable. This then enables us to efficiently and consistently estimate the deep parameters of the economic model together with their associated standard errors.

As the model is primarily to be used for forecasting and simulation, the approach to estimation was to put a stronger weight on more recent data compared to historical data. This was achieved by starting with a short sample for the dynamic equations, and then rolling the sample back to ensure no structural breaks. The table below records the starting sample for the dynamic equations. End period is 2007Q2 for all equations.

Estimation Sample

\begin{tabular}{|l|l|l|l|l|l|}
\hline Equations $\backslash$ Country & FR & DE & IT & ES & NL \\
\hline Investment & $83 q 1$ & $93 q 1$ & $89 q 1$ & $93 q 1$ & $84 q 1$ \\
\hline Employment & $82 q 3$ & $93 q 1$ & $92 q 1$ & $86 q 1$ & $93 q 1$ \\
\hline Consumption & $88 q 1$ & $82 q 1$ & $91 q 1$ & $91 q 1$ & $83 q 1$ \\
\hline Wages & $83 q 4$ & $91 q 1$ & $91 q 1$ & $90 q 1$ & $85 q 1$ \\
\hline Inflation & $84 q 4$ & $93 q 1$ & $93 q 1$ & $93 q 1$ & $91 q 1$ \\
\hline Inventories & $82 q 2$ & $82 q 1$ & $90 q 1$ & $88 q 1$ & $88 q 2$ \\
\hline Real exports & $85 q 1$ & $91 q 1$ & $92 q 1$ & $93 q 1$ & $87 q 1$ \\
\hline Real imports & $85 q 1$ & $82 q 1$ & $85 q 2$ & $84 q 1$ & $85 q 2$ \\
\hline Export prices & $85 q 1$ & $91 q 1$ & $92 q 1$ & $93 q 1$ & $87 q 1$ \\
\hline Import prices & $85 q 1$ & $91 q 1$ & $85 q 2$ & $84 q 1$ & $85 q 2$ \\
\hline
\end{tabular}

Below we report the key estimated equations and parameters, and where relevant the roots of the equations, where the closer to unity they are the more forward/backward looking is the behaviour. In the Appendix we report the instruments used and the normalized equation parameters for the forward-looking equations.

\subsubsection{Labour Demand}

From the point of view of employment, the desired (optimal) number of workers is derived from the inverted production function equation (71) such that:

$$
N_{t}^{*}=\frac{\bar{N}(1-\bar{\pi})^{\frac{\sigma}{\sigma-1}}}{\Gamma_{N}(t)}\left[\left(\frac{Y_{t}}{\xi \bar{Y}}\right)^{\frac{\sigma-1}{\sigma}}-\bar{\pi}\left(\frac{K_{t-1}}{\bar{K}}\right)^{\frac{\sigma}{\sigma-1}}\right]^{\frac{\sigma}{\sigma-1}}
$$


Changes in employment are coupled with adjustment costs. For estimation of the dynamic labour demand, we need to define function forms for the adjustment cost function $A_{N}\left(N_{t}, N_{t-1}\right)$. See equation (42)

$$
A_{N}\left(N_{t}, N_{t-1}\right)=\frac{a_{N}}{2} \cdot \Delta N_{t} \Delta n_{t}
$$

where $\mathrm{n}=\log (\mathrm{N})$.

Now the dynamic system of first order conditions imply the following labour demand:

$$
n_{t}=\frac{D_{t}}{\left(1+D_{t}+a_{h} / a_{N}\right)} n_{t+1}+\frac{1}{\left(1+D_{t}+a_{h} / a_{N}\right)} n_{t-1}+\frac{a_{h} / a_{N}}{\left(1+D_{t}+a_{h} / a_{N}\right)} n_{t}^{*}
$$

$n_{t}^{*}=\log \left(N_{t}^{*}\right)$ (inverted production function)

$$
D_{t}=\frac{\left(1+\left(w_{t+1}-w_{t}\right)\right)}{\left(1+r_{t}\right)_{t}} \cdot \frac{\left(1+\left(n_{t}^{*}-n_{t}\right)+a_{h}\left(n_{t}^{*}-n_{t}\right)^{2}\right)}{\left(1+\left(n_{t+1}^{*}-n_{t+1}\right)+a_{h}\left(n_{t+1}^{*}-n_{t+1}\right)^{2}\right)} \cdot=\operatorname{discounting~factor}(\approx 1)
$$

The estimated parameters and the implied roots of the homogenous part of the difference equation are presented in the table below. In all cases the backward root is high implying slow adjustment to shocks. However, also the inverse of forward roots are quite high, although well below unity, implying forward lookingness in labour demand. Spain is both the least backward and least forward looking country implying demand for labour is more affected by contemporaneous effects.

Labour Demand Estimation results

\begin{tabular}{|r|r|r|r|r|r|}
\hline & FR & DE & IT & ES & NL \\
\hline$a_{h} / a_{N}$ & 0.0225 & 0.0388 & 0.0559 & 0.1414 & 0.0396 \\
& $(0.004)$ & $(0.011)$ & $(0.020)$ & $(0.037)$ & $(0.008)$ \\
\hline 1/Root 1 (forward) & 0.8564 & 0.8171 & 0.7855 & 0.6848 & 0.8173 \\
\hline Root 2 (backward) & 0.8646 & 0.8248 & 0.7932 & 0.6904 & 0.8219 \\
\hline p-value of J-test & 0.85 & 0.974 & 0.84 & 0.839 & 0.955 \\
\hline
\end{tabular}

*Standard errors of estimates in brackets

\subsubsection{Capital formation}

Capital accumulation reflects time-to-build considerations. In addition to conventional demand and technology constraints the present value maximisation is constrained by non-negativity of gross investment and by the upper bound for the debt-to capital stock ratio. Hence, the estimated aggregate level equations assume that for a certain percent of firms these constraints are binding.

As with employment, we define the adjustment cost function $A\left(K_{t}, K_{t-1}, K_{t-2}\right)$, as follows:

$$
A\left(K_{t}, K_{t-1}, K_{t-2}\right)=\frac{a_{K}}{2} \cdot \Delta K_{t} \Delta k_{t}+\frac{a_{K} b_{K}^{2}}{2} \cdot \Delta K_{t-1} \Delta k_{t-1}-a_{K} b_{K} \cdot \Delta K_{t} \Delta k_{t-1}
$$

where $k=\log K$ and $b_{K} \in[0,1]$.

Now the dynamic system of first order conditions implies the investment equation: 


$$
\begin{aligned}
\frac{\left(1-\Lambda^{B}\right)^{2} b_{K}}{\left(1+r_{t}\right)\left(1+r_{t+1}\right)} \Delta k_{t+2} & -\left(\frac{\left(1-\Lambda^{B}\right)^{2} b_{K}^{2}}{\left(1+r_{t}\right)\left(1+r_{t+1}\right)}+\frac{\left(1-\Lambda^{B}\right)\left(1+b_{K}\right)}{\left(1+r_{t}\right)}\right) \Delta k_{t+1} \\
& +\left(\frac{\left(1-\Lambda^{B}\right) b_{K}\left(1+b_{K}\right)}{\left(1+r_{t}\right)}+1\right) \Delta k_{t}-b_{K} \Delta k_{t-1} \\
= & \frac{1}{a_{K}}\left(\frac{P_{t}}{\left(1+\mu_{t}\right) P_{t}^{I}} M P K_{t}-\left\{\left(1-\Lambda^{I}\right) U C_{t}+\Lambda^{B}(1-\delta)\left[\alpha-\left(1-\Lambda^{I}\right)\right]\right\}\right)
\end{aligned}
$$

$\Lambda^{B}=$ LG-multiplier related to the borrowing constraint; $\alpha=$ the debt to capital stock ceiling ratio $0 \leq \alpha \leq 1 ; M P K=$ marginal product of capital; $U C=$ real user cost of capital; $\Lambda^{I}=$ LG-multiplier related to the irreversibility of investment ; and $\mathrm{a}_{K}$ and $\mathrm{b}_{K}$ are adjustment cost parameters.

The estimated parameters and implied roots are reported in the table below. In all cases the backward root (which equals parameter $\mathrm{b}_{k}$ ) is high implying slow adjustment to shocks, however firms are also forward-looking. From the roots, we see that Spain is the most forward looking and the Netherlands the least forward looking.

Total investment has been further disaggregated via bridge equations to housing (incorporating short/long-term interest rates), and non-residential, but all feedback effects to the rest of the model go through aggregate fixed investment.

Estimated Investment parameters*

\begin{tabular}{|r|c|c|c|c|c|}
\hline & FR & DE & IT & ES & NL \\
\hline $1-\Lambda^{B}$ & 0.5087 & 0.4517 & 0.5067 & 0.5876 & 0.376 \\
& $(0.012)$ & $(0.005)$ & $(0.008)$ & $(0.011)$ & $(0.015)$ \\
\hline$b_{K}$ & 0.7879 & 0.695 & 0.7796 & 0.7834 & 0.6267 \\
& $(0.010)$ & $(0.008)$ & $(0.012)$ & $(0.014)$ & $(0.026)$ \\
\hline $1 / a_{K}$ & 0.0126 & 0.0122 & 0.0129 & 0.0139 & 0.0163 \\
& $(0.0007)$ & $(0.0006)$ & $(0.0010)$ & $(0.0016)$ & $(0.0016)$ \\
\hline$\Lambda^{I}$ & 0.25 & 0.26 & 0.2 & 0.25 & 0.33 \\
\hline$\alpha$ & 0.8 & 0.8 & 0.855 & 0.8 & 0.75 \\
\hline & & & & & \\
\hline 1/Root 1 (forward) & 0.3961 & 0.311 & 0.3918 & 0.4585 & 0.2306 \\
\hline 1/Root 2 (forward) & 0.5027 & 0.4475 & 0.5026 & 0.5852 & 0.3679 \\
\hline Root 3 (backward) & 0.7879 & 0.695 & 0.7796 & 0.7834 & 0.6267 \\
\hline p-value of J-test & 0.999 & 0.999 & 0.99 & 0.996 & 0.993 \\
\hline
\end{tabular}

${ }^{*}$ Standard errors of estimates in brackets

\subsubsection{Price formation}

Price setting are staggered with three-valued Calvo-signal resulting in a conventional hybrid New Keynesian Phillips curve as in Gali and Gertler (1999); see equation (54) without quadratic inflation terms:

$$
\begin{aligned}
\left\{\theta_{p}+\omega_{p}\left[1-\theta_{p}(1-\beta)\right]\right\} \Delta p_{t}-\omega_{p} \Delta p_{t-1}-\beta \theta_{p} \Delta p_{t+1} & \\
- & \left(1-\omega_{p}\right)\left(1-\theta_{p}\right)\left(1-\beta \theta_{p}\right)\left(p_{t}^{*}-p_{t}\right)=0
\end{aligned}
$$


where $p_{t}=\log$ of gdp deflator at factor costs; $p_{t}^{*}=w_{t}-m p n_{t}+a_{h}\left(n_{t}^{*}-n_{t}\right)+\mu_{t}=\log$ of the frictionless equilibrium price level; $w_{t}=\log$ of compensation per worker; $m p n_{t}=\log$ of the marginal product of labour (<= production function); $n_{t}^{*}=$ optimal number of workers (log), $n_{t}=$ actual employment $(\log ) ; a_{h}$ is the overtime premium parameter determined by (46) and aggregate mark-up $\mu(t)$ is determined by the system (76)-(80). $\theta_{p}$ is the probability that firms don't change their prices, and $\omega_{p}$ is the probability that prices are changed following a backward-looking rule.

For Germany and Spain the hybrid New Keynesian Phillips curve was estimated as in McAdam and Willman (2004) in the following present value form:

$$
\Delta p_{t}=\omega_{p} \Delta p_{t-1}+\frac{\left(1-\omega_{p}\right)\left(1-\theta_{p}\right)\left(1-\beta \theta_{p}\right)}{1-\left(\beta \theta_{p}\right)^{13}} \sum_{i=0}^{12}\left(\beta \theta_{p}\right)^{i} E_{t}\left(m c n_{t+i}+\mu-p_{t-1}\right)+\text { const }
$$

Estimated parameters of the hybrid NKPC*

\begin{tabular}{|r|c|c|c|c|c|}
\hline & FR & DE & IT & ES & NL \\
\hline$\theta_{p}$ & 0.7455 & 0.7646 & 0.7251 & 0.6705 & 0.6926 \\
& $(0.030)$ & $(0.009)$ & $(0.013)$ & $(0.017)$ & $(0.043)$ \\
\hline$\omega_{p}$ & 0.3531 & 0.3831 & 0.3235 & 0.2543 & 0.2807 \\
& $(0.045)$ & $(0.015)$ & $(0.018)$ & $(0.022)$ & $(0.054)$ \\
\hline$a_{h}$ & 0.7515 & 0.399 & 0.1797 & 0.4212 & 0.4109 \\
& $(0.237)$ & $(0.214)$ & $(0.222)$ & $(0.204)$ & $(0.156)$ \\
\hline & & & & & \\
\hline Duration & 3.93 & 4.25 & 3.64 & 3.03 & 3.25 \\
\hline 1/Root 1 (forward) & 0.7380 & 1.321 & 0.7179 & 0.6638 & 0.6857 \\
\hline Root 2 (backwards) & 0.597 & 0.6216 & 0.5712 & 0.5067 & 0.5298 \\
\hline Root 3 (backwards) & 0.5914 & 0.6163 & 0.5663 & 0.502 & 0.5298 \\
\hline p-value of J-test & 0.867 & 0.890 & 0.967 & 0.896 & 0.922 \\
\hline
\end{tabular}

${ }^{*}$ Standard errors of estimates in brackets

In estimation we assumed the four per cent annual discount rate, which in quarterly data implies $\beta=0.99$.

Our estimation results imply that the duration, i.e. the average time firms keep prices fixed, is between $3-5$ quarters. This range is somewhat shorter than the estimates of Gali and Gertler (1999) and Gali, Gertler and Lopez-Salido (2001), whose estimated average duration was 5 - 6 quarters, and markedly below the estimates from DSGE models of the euro area ${ }^{14}$. Our results are, however, strongly supported by a broad range of micro studies that suggest the average duration of price changes in the Euro area ranges from four to five quarters, see Álvarez et al (2006) and Altissimo, Ehrmann and Smets (2006). Some other studies suggest that average duration of price changes could be even less than 4 quarters, e.g. Eichenbaum, Jaimovich and Rebelo (2008), and Fabiani et al (2006) and possibly less than 2 quarters Dias et al (2007). Given this evidence, we consider our estimates very plausible.

Disaggregated Price Equations The GDP deflator at factor costs is an integral part of the supply side and the key determinant of other price variables in the model. In this regard, it is the central price in the model. However, adjustment in other prices also matter for differences in the models'

\footnotetext{
${ }^{14}$ For instance, Smets and Wouters (2003) estimates suggested prices change once every two and a half years and Christoffel, Coenen and Warne (2008) estimations imply even longer duration.
} 
response. Below we present the estimation results for Harmonized consumer price indices (HICP), which are represented by two equations, one for the energy HICP and one for non-energy HICP.

The post-tax HICP deflator is defined as:

$$
p_{t}^{H X}=\frac{1-t c i r}{1-t c i_{t}} p_{t}^{H X T}
$$

where $\mathrm{p}_{t}^{H X T}$ is the pre-tax HICP excluding energy, tci is the current implicit tax rate and tcir is the tax rate in the base year of price indices. We model the seasonal adjusted version of $\mathrm{p}_{t}^{H X T}$, so-called $\mathrm{p}_{t}^{H X S T}$ where the seasonal factors are estimated using a time-varying airline estimation procedure and kept fixed over the forecast horizon. We retain the Calvo price framework and parameters from above:

$$
\begin{aligned}
\left\{\theta_{p}+\omega_{p}\left[1-\theta_{p}(1-\beta)\right]\right\} \Delta p_{t}^{H X S T}- & \omega_{p} \Delta p_{t-1}^{H X S T}-\beta \theta_{p} \Delta p_{t+1}^{H X S T} \\
& -\left(1-\omega_{p}\right)\left(1-\theta_{p}\right)\left(1-\beta \theta_{p}\right)\left(p_{t}^{H X S T *}-p_{t}^{H X S T}\right)=0
\end{aligned}
$$

where $\mathrm{p}_{t}^{H X S T *}$ is the long-run optimal non-energy HICP and is weighted average of the optimal GDP deflator $\mathrm{p}_{t}^{*}$ including indirect energy prices and imports deflator excluding energy, $p_{t}^{M N}$, where the weights $\phi_{1}$ are estimated by OLS and $\varrho$ is set to 0.015 based on input-output tables. In addition, as with the Calvo price equation, we include a labour adjustment factor:

$$
p_{t}^{H X S T *}=\phi_{1} p_{t}^{M N}+\left(1-\phi_{1}\right)\left((1-\varrho) p_{t}^{*}+\varrho p_{t}^{E I}\right)+a_{h}\left(n_{t}^{*}-n_{t}\right)
$$

HICP energy $\left(\mathrm{P}_{t}^{H E}\right)$ is modelled as a mark-up of energy prices (or oil) and GDP deflator $\left(\mathrm{p}_{t}\right)$ :

$$
p_{t}^{H E}=\delta_{1} p_{t}^{E I}+\left(1-\delta_{1}\right)\left(p_{t}\right)
$$

HICP energy and excluding energy and taxes seasonally adjusted

\begin{tabular}{|c|c|c|c|c|c|}
\hline & DE & FR & IT & ES & NL \\
\hline$\phi_{1}$ & 0.21 & 0.15 & 0.1 & 0.033 & 0.17 \\
& $(0.071)$ & $\cdot$ & $(0.014)$ & $(0.005)$ & $(0.018)$ \\
\hline$\delta_{1}$ & 0.33 & 0.21 & 0.15 & 0.21 & 0.35 \\
& $(0.07)$ & $(0.098)$ & $(0.006)$ & $(0.008)$ & $(0.011)$ \\
\hline
\end{tabular}

*Standard errors of estimates in brackets

The overall HICP $p_{t}^{H}$,then becomes a weighted average of HICP non-energy (post-tax), $p_{t}^{H X}$ and HICP energy, $p_{t}^{H E}$ where $w_{e t}$ is the weight of HICP energy in the overall HICP ${ }^{15}$.

$$
p_{t}^{H}=w_{e t} \cdot p_{t}^{H E}+\left(1-w_{e t}\right) \cdot p_{t}^{H X}
$$

The consumption deflator is linked via a simple bridge equation to seasonally adjusted HICP.

All other domestic deflators (e.g. investment deflator) are specified as quasi-identities, i.e. modelled as weighted averages of domestic costs (measured by the value-added deflator defined above) and import prices (measured by the import deflator). This feature ensures static homogeneity in all price equations. For pre-tax deflators we assume that imports are 'cost, insurance and freight at the

\footnotetext{
${ }^{15}$ In 2004 energy HICP weight varied across the countries in the range of 8-10 per cent
} 
importer's border' (cif) and the exports are 'free on board at the exporter's border' (fob). For this reason indirect taxes are levied only on total consumption (private and public) and total investment. Since there is no distinction between indirect taxes on consumption goods and on investment goods, both tax rates will be equal in sample but will be kept under different denominations for simulation purposes.

\subsubsection{Wage Setting}

As with the price setting, wages are also set via a staggered with three-valued Calvo-signal where part of unions keep wages fixed, $\theta_{w}$, another part changes wages following backward-looking rule, $\omega_{w}$, and the rest set them optimally ${ }^{16}$ :

$$
\begin{aligned}
\left\{\theta_{w}+\omega_{w}\left[1-\theta_{w}(1-\beta)\right]\right\} & \Delta w_{t}=\omega_{w} \Delta w_{t-1}+\beta \theta_{w} E_{t} w_{t+1} \\
+ & \left(1-\omega_{w}\right)\left(1-\theta_{w}\right)\left(1-\beta \theta_{w}\right)\left\{w_{t}^{*}-w_{t}\right\}
\end{aligned}
$$

where $w_{t}=\log$ of compensation per worker, and $\beta$, the discount factor, $=0.99$. The optimal frictionless wage rate, $w_{t}^{*}$ is based on two alternative behavioural assumptions where we assume that part of the unions are utilitarian, maximising the utility of member households, whist the rest are non-utilitarian keeping wage development in line with productivity development coupled with a high desired employment rate. i.e.

$$
\begin{gathered}
w_{t}^{*}=a_{w u}\left[\left(p_{t}^{C}+c_{t}-n_{t}^{F}\right)-\log \left(\sigma-1+\frac{F_{N}^{C E S}}{Y_{t} / N_{t}}\right)+\log \frac{\sigma \kappa}{\bar{h}(\text { time })}\right] \\
+\left(1-a_{w u}\right)\left[p_{t}+\log \left(\frac{F_{N}^{C E S}}{1+\mu}\right)+\chi \log \left(\frac{F^{-1}\left(K_{t}, Y_{t}\right)}{\varpi \cdot N_{t}^{F}}\right)\right] \\
F_{N}^{C E S}=\left(1-\pi_{0}\right)\left(\frac{Y_{0}}{N_{0}} \Gamma_{N}\left(t, t_{0}\right)\right)^{\frac{\sigma-1}{\sigma}}\left(\frac{Y_{t}}{N_{t}}\right)^{\frac{1}{\sigma}}
\end{gathered}
$$

where $c_{t}=$ consumption $(\log ), p_{t}^{C}=$ consumption deflator $(\log ) ; F^{-1}(\cdot)=$ inverted CES production function ( desired number of workers); $N_{t}^{F}=$ labour force and the gap between optimal labour demand and supply measures the wage drift effect.

Note that if all unions are non-utilitarian, then in the full model context, it implies a constant long-run natural rate of unemployment. If instead, all or part of the unions are utilitarian, then shocks that affect the demand structure, e.g. a permanent government expenditure shock affecting the GDP share of private consumption, affect also the long-run equilibrium unemployment rate.

The weight parameter $a_{w u}$ is estimated in the context of the estimation of equation (93). Naturally, for scenario analysis its value can be imposed to unity or zero, depending if we want to assume all unions to be utilitarian or non-utilitarian ${ }^{17}$.

\footnotetext{
${ }^{16}$ As for prices (see equation 87), in Germany we have followed the present value form of this equation.

${ }^{17}$ For the simulations in the paper we assume all unions to be non-utilitarian.
} 
Estimated parameters of the hybrid wage-NKPC*

\begin{tabular}{|r|c|c|c|c|c|}
\hline & $\mathrm{FR}$ & $\mathrm{DE}$ & $\mathrm{IT}$ & $\mathrm{ES}$ & $\mathrm{NL}$ \\
\hline$\theta_{w}$ & 0.7364 & 0.7982 & 0.7301 & 0.7471 & 0.7077 \\
& $(0.028)$ & $(0.025)$ & $(0.024)$ & $(0.021)$ & $(0.022)$ \\
\hline$\omega_{w}$ & 0.3396 & 0.4412 & 0.3306 & 0.3555 & 0.2999 \\
& $(0.041)$ & $(0.047)$ & $(0.034)$ & $(0.032)$ & $(0.029)$ \\
\hline$\chi$ & 0.1 & 0.2 & 0.1 & 0.15 & 0.11 \\
& - & - & - & - & - \\
\hline$a_{w u}$ & 0.3 & 0.15 & 0.5366 & 0.2234 & 0.1745 \\
& - & - & $(0.049)$ & $(0.061)$ & $(0.048)$ \\
\hline & & & & & \\
\hline 1/Root 1 (forward) & 0.7290 & 0.7903 & 0.7228 & 0.7396 & 0.7006 \\
\hline Root 2 (backwards) & 0.5591 & 0.6683 & 0.5769 & 0.5963 & 0.5488 \\
\hline Root 3 (backwards) & 0.5561 & 0.6603 & 5730 & 0.5963 & 0.5465 \\
\hline p-value of J-test & 0.914 & 0.844 & 0.912 & 0.775 & 0.983 \\
\hline
\end{tabular}

${ }^{*}$ Standard errors of estimates in brackets

In the table we report the average duration between wage changes. Estimated wage durations are in all countries around 4 quarters. Existing information on the frequency of wage changes is rather scarce, but recent finding of the Wage Dynamic Network (2010) based on survey evidence suggested average duration of wages is about 5 quarters, see Druant et al. (2009), compared to an average duration of prices of slightly above 3 quarters. This finding also seems consistent with estimates of average contract length in collective wage agreements (of between one and 1.5 years). For the euro area, DSGE models, both standard ones like Smets and Wouters (2003) and Christoffel, Coenen and Warne (2008) and ones with more detailed labour markets e.g. De Walque et al (2010), find the average duration of wage contracts is estimated to be just over one year. Hence, our estimates are broadly within the range of estimates and similar to the price duration effects, Taylor (1999).

\subsubsection{Inventory investment}

Following general practice and to neglect excessive complexity of the profit maximization framework, inventory formation is left outside that framework. Instead inventory formation is derived on the basis of second stage optimization by assuming that firms minimise a quadratic loss function specified in terms of the deviations of inventories from the optimal level, on one hand, and the deviations of output (sales of storable goods) from the level corresponding to the optimal use of existing input.

The desired equilibrium inventory stock $\mathrm{KII}^{*}$ is based on the estimated CES production function:

$$
K I I=a+b F(K, N, t)
$$

and inventories from the dynamic equation:

$$
\begin{aligned}
& (1-r \cdot A) \Delta K I I_{t}=(1-2 A) \Delta K I I_{t}^{*} \\
& \quad-A\left[\Delta S_{t}-\Delta K I I_{t}-\Delta F(\cdot)\right]+(1-r) A\left[\Delta S_{t+1}-\Delta K I I_{t+1}-\Delta F(\cdot, t+1)\right]
\end{aligned}
$$

$\mathrm{S}=$ Sales (Private consumption + exports) 
Inventory Estimation results*

\begin{tabular}{|r|c|c|c|c|c|}
\hline & FR & DE & IT & ES & NL \\
\hline$A$ & 0.4705 & 0.3374 & 0.3501 & 0.3038 \\
$(0.027)$ & $(0.032)$ & $(0.072)$ & $\begin{array}{c}0.3802 \\
(0.058)\end{array}$ & $(0.047)$ \\
\hline & & & & & \\
\hline 1/Root 1 (forward) & 0.7372 & 0.3855 & 0.4055 & 0.3361 & 0.4579 \\
\hline Root 2 (backward) & 0.7447 & 0.3893 & 0.4096 & 0.3395 & 0.4625 \\
\hline p-value of J-test & 0.731 & 0.889 & 0.92 & 0.972 & 0.987 \\
\hline
\end{tabular}

${ }^{*}$ Standard errors of estimates in brackets

Estimation results indicate that all 5-countries inventories show quite fast adjusted to their optimal level. The adjustment process is fastest in Spain and slowest in France.

\subsection{Households}

The optimization framework of households is Blanchard's (1985) overlapping generation model with perpetual youth, where risk-averse consumers maximize their life-time utility under imperfect (frontloaded) information accounting for internal habit formation. This results in a forward looking aggregate consumption function with strong backward-looking frictional elements, see equation (23). In the estimated specification we assume that the subjective discount rate $\rho=\bar{r}=0.01\left(\right.$ and $\bar{R}=(1-\bar{r})^{-1}$ ) which corresponds to an annual rate of 4 percent. Further, to eliminate heteroscedasticity we divide both sides of equations by current period labour income. Hence, in estimating the equation with the method of instrumental variables it can be written as follows:

$$
E_{t}\left\{\begin{array}{c}
\left\{1+\frac{\gamma}{1.01}\left[(1-\pi)^{2} a R_{t}-(0.01+\pi)\left(1-\frac{(1-\pi) a}{1.01}\right)\right]\right\} \\
\frac{C_{t}}{Y_{t}}-(1-\pi) \gamma \frac{R_{t} C_{t+1}}{Y_{t}}-a(1-\pi) \frac{C_{t-1}}{Y_{t}}-\left(\frac{1.01-(1-\pi) a}{1.01}\right)\left(\frac{0.01+\pi}{1.01}\right) \\
\left\{\left(\frac{1}{1-\pi}-\gamma\right)\left(\frac{V_{t-1}}{Y_{t}}+1\right)+\left(\frac{\Lambda(1.01-(1-\pi) \gamma)}{0.01+\pi}-\frac{1}{1-\pi}\right)\right\} z_{t}
\end{array}\right\}=0
$$

where $\mathrm{C}_{t}$ is consumption, $\mathrm{Y}_{t}$ labour income net of payroll taxes minus transfers, $V_{t-1}$ total wealth in the beginning of period. Parameter $\pi=$ death probability; $\gamma=$ forward information parameter; $a=$ habit persistence parameter; $\Lambda=$ income risk parameter, and $z_{t}$ refers to the set of instruments.

As firms are owned by households, total private sector wealth can be used as operational counterparty of $V_{t-1}$ in (98). In a macroeconomic framework the advantage of this large wealth concept is that its component corresponding to accumulated savings equals the sum of the private sector capital stock (at repurchasing prices), net government debt GDN and net foreign assets NFA. However, as also changes in real asset prices may affect the "perceived" wealth relevant for consumption decisions, $V_{t-1}$ is operationalised as follows:

$$
V_{t-1}=\frac{P_{I}}{P_{C}}\left[\begin{array}{c}
\left(\frac{P_{S}}{P_{I}}\right)^{b 0}\left(1-s_{H}\right)\left(K S R_{t-1}-K G R_{t-1}\right)+ \\
\left(\frac{P_{H}}{P_{I}}\right)^{b 1} s_{H}\left(K S R_{t-1}-K G R_{t-1}\right)
\end{array}\right]+\frac{G D N_{t-1}+N F A_{t-1}}{P_{C}}
$$

where $P_{I}, P_{C}, P_{S}$ and $P_{H}$ are investment deflator, consumption deflator, stock prices and the market price of housing, respectively. KSR is total capital stock, KGR is government sector capital stock and $s_{H}$ is the share of housing stock of total private capital stock. Now, if elasticity parameters $b 0$ and $b 1$ equal zero, then asset prices have no effects on consumption and "perceived" wealth equals total private sector wealth at repurchasing prices. 
Correspondingly, if $b 0=b 1=1$, then variations in stock and housing prices are fully transmitted into the "perceived" wealth with maximal effects on consumption. These parameters were estimated jointly with other parameters of the dynamic consumption function specified for the five biggest euro area countries. In estimated equations $b 0$ and $b 1$ were constrained to be equal, because data was not able to credibly identify possible differentiated elasticity effects. These equations implied the following long-run marginal properties to consume out of wealth $\left(\mathrm{mcr}_{w}\right)$ and labour income $\left(m c r_{y}\right)$ as well as elasticity parameters $(b 0$ and $b 1)$.

\section{Consumption equation*}

\begin{tabular}{|r|c|c|c|r|r|}
\hline & FR & DE & IT & ES & NL \\
\hline$\pi$ & 0.005 & 0.007 & 0.0053 & 0.0053 & 0.0053 \\
\hline$\gamma$ & 0.8138 & 0.7923 & 0.6144 & 0.7192 & 0.6665 \\
& $(0.138)$ & $(0.004)$ & $(0.055)$ & $(0.09)$ & $(0.052)$ \\
\hline $\mathrm{a}$ & 0.9303 & 0.8715 & 0.9467 & 0.864 & 0.9098 \\
& $(0.036)$ & $(0.004)$ & $(0.012)$ & $(0.069)$ & $(0.013)$ \\
\hline$\Lambda$ & 0.9031 & 0.8002 & 0.6711 & 0.8522 & 0.7196 \\
& $(0.038)$ & $(0.004)$ & $(0.021)$ & $(0.017)$ & $(0.013)$ \\
\hline $\mathrm{b} 0, \mathrm{~b} 1$ & - & 0.2828 & 0.2036 & 0.2388 & 0.336 \\
& & $(0.024)$ & $(0.101)$ & $(0.1)$ & $(0.048)$ \\
\hline & & & & & \\
\hline $\mathrm{mcr}_{w}$ & 0.06 & 0.07 & 0.06 & 0.06 & 0.06 \\
\hline $\mathrm{mcr}_{y}$ & 0.91 & 0.79 & 0.61 & 0.71 & 0.67 \\
\hline 1/Root 1 (forward) & 0.805 & 0.783 & 0.606 & 0.711 & 0.658 \\
\hline Root 2 (backward) & 0.92 & 0.862 & 0.934 & 0.854 & 0.898 \\
\hline p-value of J-test & 0.908 & 0.949 & 0.701 & 0.946 & 0.831 \\
\hline
\end{tabular}

*Standard errors of estimates in brackets

The long-run marginal propensity to consume out of total wealth is, according to these estimates, 7 cents per euro in Germany and 6 cents in the other four countries. Positive stock and housing price effects on consumption were estimated for all countries except for France (where they were not found to be significant). According to these estimates across countries, in the long-run about 20-34 percent of changes in stock and housing prices are transmitted to the respective wealth components and further to consumption.

\subsection{External Sector Behaviour}

\subsubsection{Export formation}

Profit maximisation under the AIDS type of export demand function results in the following 2-equation system for the export volume and export price :

$$
\begin{gathered}
\left(\frac{P_{X} X}{P_{c x} M F}\right)=a+b \cdot f(\text { time })-(\phi-1)\left(p_{X}-p_{C X}\right) \\
p_{X}=a+\frac{1+(a+b \cdot f(\text { time })) /(\phi-1)}{2+(a+b \cdot f(\text { time })) /(\phi-1)}\left(\left(1-a_{x}\right)(w-m p n)+a_{x} p_{M}\right) \\
+\frac{1}{2+(a+b \cdot f(\text { time })) /(\phi-1)} p_{C X}
\end{gathered}
$$


Where $P_{X}=$ Export deflator (lower case refers to $\log$ ); $X=$ Export volume; $P_{C X}=$ the external competitor export prices (lower case refers to $\log$ ); $M F=$ the world demand for exports; $w=$ compensation per worker $(\log ) ; m p n=$ marginal product of labour $(\log ) ; p_{M}=$ import deflator $(\log ), a=$ point market share (with indexed data close to unity); $\phi>1$ is the representative point price elasticity of exports; $b$ if different from zero measures the deviation of income elasticity of export demand from unity; and $a_{X}=$ import content of exports (input-output estimate). In estimation an additional free trend variable was allowed in the price equation.

2-equation export system estimates*

\begin{tabular}{|r|c|c|c|c|c|}
\hline & DE & FR & IT & ES & NL \\
\hline$a$ & 1.08 & 1.131 & 1.051 & 1.05 & 1.025 \\
& $(0.01)$ & $(0.01)$ & $(0.01)$ & $(0.01)$ & $(0.01)$ \\
\hline$\varnothing$ & 1.021 & 1.056 & 1.220 & 1.314 & 1.345 \\
& $(0.03)$ & $(0.06)$ & $(0.09)$ & $(0.09)$ & $(0.07)$ \\
\hline $\mathrm{a}_{X}$ & 0.385 & 0.167 & 0.400 & 0.465 & 0.672 \\
\hline Log-det & -16.49 & -16.83 & -14.61 & -16.65 & -14.49 \\
\hline ADF volume-eq. & -3.81 & -4.954 & -2.095 & -3.16 & -3.798 \\
\hline ADF price-eq. & -3.735 & -2.812 & -2.918 & -4.0 & -2.482 \\
\hline
\end{tabular}

${ }^{*}$ Standard errors of estimates in brackets

The dynamic export volume and export price equations follow conventional error correction equations for the log change in exports or export prices.

Dynamic export equation estimates*

\begin{tabular}{|c|c|c|c|c|c|}
\hline & $\mathrm{DE}$ & FR & IT & ES & NL \\
\hline$M F$ & $\begin{array}{c}0.846 \\
(0.182)\end{array}$ & $\begin{array}{l}0.745 \\
(0.11)\end{array}$ & $\begin{array}{c}0.663 \\
(0.171)\end{array}$ & $\begin{array}{c}0.68 \\
(0.186)\end{array}$ & $\begin{array}{c}0.926 \\
(0.078)\end{array}$ \\
\hline$P_{C X} / P_{X}$ & $\begin{array}{l}-0.288 \\
(0.154)\end{array}$ & $\begin{array}{c}-0.239 \\
(0.1)\end{array}$ & $\begin{array}{l}-0.573 \\
(0.093)\end{array}$ & $\begin{array}{l}-0.399 \\
(0.19)\end{array}$ & $\begin{array}{l}-0.285 \\
(0.087)\end{array}$ \\
\hline$\Delta x_{t-1}^{*}$ & - & - & - & $\begin{array}{l}0.271 \\
(0.12)\end{array}$ & - \\
\hline$E C-$ term & $\begin{array}{l}-0.152 \\
(0.06)\end{array}$ & $\begin{array}{l}-0.42 \\
(0.06)\end{array}$ & $\begin{array}{l}-0.157 \\
(0.053)\end{array}$ & $\begin{array}{l}-0.165 \\
(0.091)\end{array}$ & $\begin{array}{l}-0.088 \\
(0.042)\end{array}$ \\
\hline R-square & 0.41 & 0.559 & 0.486 & 0.281 & 0.378 \\
\hline $\mathrm{D}-\mathrm{W}$ & 2.218 & 1.948 & 1.775 & 1.873 & 2.174 \\
\hline \multicolumn{6}{|c|}{ Dynamic export price equation } \\
\hline \multirow[t]{2}{*}{$\Delta P^{x *}$} & - & 0.795 & 0.529 & 0.726 & 0.930 \\
\hline & & $(0.082)$ & $(0.096)$ & $(0.097)$ & $(0.109)$ \\
\hline \multirow[t]{2}{*}{$\Delta P_{t-1}^{x *}$} & - & 0.177 & - & - & - \\
\hline & & $(0.080)$ & & & \\
\hline \multirow[t]{2}{*}{$\Delta P_{t-1}^{x}$} & - & - & 0.266 & - & - \\
\hline & & & $(0.070)$ & & \\
\hline \multirow[t]{2}{*}{$\Delta P_{t-2}^{x}-\Delta P_{t-3}^{x}$} & 0.1333 & - & - & - & - \\
\hline & $(0.0353)$ & & & & \\
\hline \multirow[t]{2}{*}{$E C-$ term } & -0.328 & -0.097 & -0.171 & -0.548 & -0.11 \\
\hline & $(0.054)$ & $(0.048)$ & $(0.061)$ & $(0.113)$ & $(0.049)$ \\
\hline R-square & 0.449 & 0.633 & 0.468 & 0.663 & 0.489 \\
\hline $\mathrm{D}-\mathrm{W}$ & 1.343 & 1.984 & 2.005 & 1.906 & 2.002 \\
\hline
\end{tabular}




\subsubsection{Import formation}

As mentioned above, the modelling approach of imports is conventional where the import supply curve is assumed to be horizontal and, hence, import volume is demand determined. Accordingly two driving variables are the domestic demand indicator and the relative price of domestic production and imports. The demand indicator $\mathrm{e}_{M R}$ is the demand indicator for imports (import content weighted index of domestic demand) derived from input-output tables:

\section{Import Content of Demand}

\begin{tabular}{|r|r|r|r|r|r|}
\hline & DE & FR & IT & ES & NL \\
\hline Consumption & 0.211 & 0.197 & 0.21 & 0.194 & 0.319 \\
\hline Investment & 0.261 & 0.243 & 0.286 & 0.285 & 0.523 \\
\hline Government & 0.056 & 0.076 & 0.055 & 0.075 & 0.107 \\
\hline Stocks & 0.508 & 0.327 & 0.437 & 0.482 & 0.7 \\
\hline Exports & 0.385 & 0.166 & 0.4 & 0.465 & 0.672 \\
\hline
\end{tabular}

Hence, we specify the long-run equilibrium aggregate demand for imports to depend on the demand indicator (with unit elasticity), the ratio of exports to the demand indicator and the relative domesticto-import price:

$$
m^{*}-e_{M R}=k\left(p_{M D}-p_{M N}\right)+b\left(x-e_{M R}\right)
$$

$\mathrm{P}_{M D}$ is domestic prices (gdp deflator); and $\mathrm{P}_{M N}$ is import prices excluding energy. All variables in equation (102) are measured in logs. The dynamic equation follows a standard EC specification.

Import equation*

\begin{tabular}{|c|c|c|c|c|c|}
\hline & $\mathrm{DE}$ & FR & IT & ES & NL \\
\hline$k$ & $\begin{array}{l}-0.782 \\
(0.01)\end{array}$ & $\begin{array}{l}-0.711 \\
(0.01)\end{array}$ & -1.001 & $\begin{array}{l}-0.979 \\
(0.04)\end{array}$ & $\begin{array}{l}-0.576 \\
(0.02)\end{array}$ \\
\hline$b$ & $\begin{array}{l}0.312 \\
(0.01)\end{array}$ & $\begin{array}{c}0.284 \\
(0.004)\end{array}$ & - & $\begin{array}{l}0.293 \\
(0.01)\end{array}$ & $\begin{array}{c}0.072 \\
(0.002)\end{array}$ \\
\hline Constant & $\begin{array}{l}-0.772 \\
(0.04)\end{array}$ & $\begin{array}{l}-0.558 \\
(0.04)\end{array}$ & $\begin{array}{l}-0.47 \\
(0.01)\end{array}$ & $\begin{array}{l}0.223 \\
(0.03)\end{array}$ & $\begin{array}{l}-0.522 \\
(0.01)\end{array}$ \\
\hline $\mathrm{R}$-square & 0.974 & 0.982 & 0.793 & 0.981 & 0.936 \\
\hline $\mathrm{ADF}$ & -3.981 & -3.704 & -4.124 & -3.207 & -4.032 \\
\hline \multicolumn{6}{|c|}{ Dynamic Import equation } \\
\hline$\Delta m^{*}$ & - & $\begin{array}{c}0.813 \\
(0.1)\end{array}$ & $\begin{array}{l}0.568 \\
(0.09)\end{array}$ & $\begin{array}{c}0.561 \\
(0.08)\end{array}$ & $\begin{array}{c}0.722 \\
(0.1)\end{array}$ \\
\hline$\Delta m_{t-2}$ & - & - & $\begin{array}{c}0.132 \\
(0.09)\end{array}$ & - & - \\
\hline$\Delta e_{M R}$ & $\begin{array}{l}0.642 \\
(0.08)\end{array}$ & - & - & - & - \\
\hline$E C-$ term & $\begin{array}{l}-0.235 \\
(0.06)\end{array}$ & $\begin{array}{l}-0.121 \\
(0.05)\end{array}$ & $\begin{array}{l}-0.117 \\
(0.05)\end{array}$ & $\begin{array}{l}0.164 \\
(0.05)\end{array}$ & $\begin{array}{l}-0.229 \\
(0.07)\end{array}$ \\
\hline $\mathrm{R}$-square & 0.446 & 0.402 & 0.339 & 0.394 & 0.35 \\
\hline D-W & 1.825 & 1.644 & 1.901 & 1.827 & 2.098 \\
\hline
\end{tabular}

*Standard errors of estimates in brackets

Import price equation The equation for the import deflator excluding energy is also quite traditional. It depends on the GDP deflator net of indirect taxes, TX, to capture possible pricing to market effects, and the competitors' import price $p_{C M}$ with static homogeneity condition imposed 


$$
p_{M N}=\phi_{1}\left(p+\log \left(1-T X_{1}\right)\right)+\left(1-\phi_{1}\right) p_{C M}
$$

In addition a trend term was included. The dynamic equation follows a standard EC specification. Import price (excluding energy equation)*

\begin{tabular}{|r|r|c|c|c|c|}
\hline & $\mathrm{DE}$ & $\mathrm{FR}$ & $\mathrm{IT}$ & $\mathrm{ES}$ & $\mathrm{NL}$ \\
\hline$\phi_{1}$ & 0.333 & 0.416 & 0.225 & 0.312 & 0.667 \\
\hline $1-\phi_{1}$ & 0.667 & 0.584 & 0.775 & 0.688 & 0.333 \\
& $(0.03)$ & $(0.03)$ & $(0.05)$ & $(0.05)$ & $(0.05)$ \\
\hline R-square & 0.957 & 0.99 & 0.77 & 0.991 & 0.405 \\
\hline D-W & 0.561 & 0.296 & 0.389 & 0.741 & 0.359 \\
\hline Dynamic Import price equation \\
\hline$\Delta p_{M N}^{*}$ & 0.498 & 0.673 & 0.427 & 0.669 & 0.74 \\
& $(0.07)$ & $(0.11)$ & $(0.14)$ & $(0.15)$ & $(0.18)$ \\
\hline$\Delta p_{M N, t-1}^{*}$ & 0.344 & 0.237 & - & - & - \\
\hline & $(0.08)$ & $(0.14)$ & & & \\
\hline$\Delta p_{M N, t-2}^{*}$ & 0.179 & - & - & - & - \\
& $(0.07)$ & & & & \\
\hline$\Delta p_{M N, t-1}$ & - & 0.189 & 0.158 & - & 0.26 \\
\hline & \multicolumn{7}{|c|}{$(0.10)$} & $(0.09)$ & & $(0.10)$ \\
\hline$E C-$ term & -0.118 & -0.148 & -0.235 & -0.417 & -0.16 \\
& $(0.06)$ & $(0.05)$ & $(0.06)$ & $(0.08)$ & $(0.06)$ \\
\hline $\mathrm{R}$-square & 0.649 & 0.487 & 0.243 & 0.375 & 0.30 \\
\hline D-W & 1.978 & 2.167 & 1.968 & 2.053 & 2.039 \\
\hline
\end{tabular}

Standard errors of estimates in brackets

The trade balance and net factor income equal the current account balance, which in turn is cumulated to give the stock of net foreign assets. Disaggregated trade (intra and extra) imports, exports both real and prices are modelled via bridge equations.

\subsection{Government, Central Banks and Financial Markets}

We close the model with the inclusion of the Central Bank which sets monetary policy, governments with a fiscal policy reaction function, and financial markets which are forward-looking and determine exchange rates and long-term interest rates. Although the activation of a monetary policy rule, a fiscal policy rule and an exchange rate UIP rule is required for long-run stability, there are scenarios where the model can be simulated when these aspects are exogenised (e.g. under a scenario of monetary accommodation).

\subsubsection{Governments}

The fiscal block of the model comprises a set of identities in expenditure and revenue categories. Government receipts are split into a number of components: direct taxes on households earned income $\left(\mathrm{T}_{f}\right)$ which includes social contributions split into employers 'and employee's and is determined via a fiscal rule (see below); direct taxes on firms $\left(\mathrm{T}_{o}\right)$, and indirect taxes $\left(\mathrm{T}_{I}\right)$, which include VAT and excises duties are calculated as exogenous implicit tax rates, and other public income $\left(\mathrm{OI}_{G}\right)$, which includes the gross operating surplus. On the expenditure side, the fiscal authority has (net) transfers $\left(T R_{F}\right)$, which includes pensions and unemployment payments. As these vary significantly 
over the business cycle, transfers as a proportion of nominal GDP (tr) are modelled as a function of the unemployment rate, mainly reflecting the dependency of unemployment compensation to unemployed:

$$
T R_{F}=\operatorname{tr} \cdot P Y-\varkappa W\left(N-N_{b a s}\right)
$$

where $\varkappa$ is calibrated to 0.7 . This means transfers are counter-cyclical - increasing with the unemployment rates. In addition, the fiscal authority has net interest payments on government debt $\left(\mathrm{IN}_{G}\right)$ and different types of primary expenditure categories, namely, government consumption $\left(\mathrm{G}_{N}\right)$ and government investment $\left(\mathrm{I}_{N G}\right)$ which are exogenous in real terms but can be shocked as part of a fiscal policy expansion. Finally, the government consumption deflator follows both the price of home produced goods with a weight of $\delta^{G}$ and of imported goods with a weight of $\left(1-\delta^{G}\right)$.

The public deficit (D) each period is then the difference between the receipts and expenditures:

$$
D=T_{F}+T_{o}+T_{I}+O I_{G}-T F_{F}-I N_{G}-G_{N}-I_{N G}
$$

The fiscal authority's is faced by a budget constraint which says that public debt $\mathrm{B}_{t}$ is the cumulative sum of past public deficits (D) i.e.

$$
B_{t}=B_{t-1}+D_{t}
$$

As households are non-Ricardian, the path of government debt and taxes matter for the evolution of the economy. Therefore, governments aim to insure stability of the public debt stock. This is modelled via a fiscal policy rule based on a reaction of personal income taxes to the deviation of the government's debt to GDP ratio from its predetermined target and which contributes to adjustment towards the stock-flow equilibrium in the long-run. ${ }^{18}$

$$
\Delta \tau_{t}=\varphi_{1}\left(b_{t-1}-\bar{b}\right)+\varphi_{2} \Delta b_{t-1}
$$

where $\tau_{t}$ is the personal income tax rate $\left(\mathrm{T}_{F} / Y\right)$, and $b_{t}$ is the government debt to GDP ratio $(\mathrm{B} / \mathrm{Y})$, and $\bar{b}$ is the target. The parameters $\varphi_{1}$ and $\varphi_{2}$ are set at 0.003 and 0.03 respectively.

\subsubsection{Monetary Authority}

Households and firms adjust their plans by taking into account the expected response by monetary authorities. The endogenous monetary policy rule provides the nominal anchor to the model and incorporate a smooth interest rate reaction to shocks in the short-run. The monetary policy rule follows a simple Taylor rule specification as in Clarida, Gali and Gertler (2000), in which the short term interest rate is determined by the inflation gap, where the inflation gap measures the distance between the actual inflation rate and its target level and the output gap $\left(y_{t}-\bar{y}_{t}\right)$ along with the lagged interest effect. This monetary policy rule means that the interest rate converges towards its long term target as inflation converges and the output gap closes. For the purpose of the simulations this target level of inflation $\left(\Delta \hat{\bar{p}}_{t}\right)$ is set to 2 per cent per annum, but it could, in principle, be set to any other reasonable level.

$$
i_{t}=(1-0.25) * i_{t-1}+0.25 *\left(4 * 1.5 *\left(\Delta p_{t+1}-\Delta \hat{\bar{p}}_{t}\right)+0.5 *\left(y_{t}-\bar{y}_{t}\right)\right)+\varepsilon_{t}
$$

where $i_{t}$ is the nominal interest rate and $\varepsilon_{t}$ is a serially uncorrelated shock to the interest rate. Regarding the choice of parameters, they are calibrated on the basis of available relevant estimates

\footnotetext{
${ }^{18}$ As the focus in the govenment sector stability target is more in the long than short-run and to strengthen the short run effects of fiscal policy, the fiscal policy reaction function could have longer lags from the debt ratio to the change of the income tax rate, but this would also increase the cyclicality of the model.
} 
in the literature and with the view of ensuring a sensible profile of the models' dynamic behaviour ${ }^{19}$. In particular, the parameter governing the speed of reaction to the interest rate gap is set to 0.25 . Following general convention, the inflation gap and output gap parameters are set to 1.5 and 0.5 respectively.

\subsubsection{Financial Markets}

Financial markets are forward-looking. The specification of the long-term interest rate equation is:

$$
l_{t}=0.9 * l_{t+1}+0.1 * i_{t}
$$

The exchange rate follows a standard real UIP equation:

$$
e_{t}=e_{t+1}+\left(r_{t}^{f}-r_{t}\right) / 400
$$

where $e$ is the (log of) the real exchange rate and $r_{t}$ and $r_{t}^{f}$ are the domestic and foreign real interest rates respectively.

\section{Rational Expectations}

We are able to simulate the model under different expectation formations, but in this paper we consider the case where expectations are assumed to be fully model consistent forward-looking, rational expectations, where the expected value is simply replaced by the model future realisations and the model solved iteratively, such that the expectations are fully model consistent. This means that agents, both private and government, are able to re-optimize his or her behaviour in advance taking into account the past, current and future anticipated shocks hitting the economy. Hence, expected outcomes are replaced with model outcomes i.e:

$$
y_{t+1}^{e}=y_{t+1}
$$

We solve the model in a deterministic framework, ${ }^{20}$ so that there is full information, and perfect foresight. Given that the model has significant non-linearities, we use a stacked-time Newton-Raphson algorithm where we can use a partially solved-out version as the whole model is essentially written in terms of observables. This approach has the advantage of not needing to explicitly linearize the model, and is therefore accurate when the economies are not operating close to the steady-state (e.g. during the financial crisis of 2007-2009) ${ }^{21}$ and is fast and robust compared to many first-order algorithms, see Juillard et al (1998). ${ }^{22}$ However, one drawback of this approach is that the terminal conditions must be explicitly specified. We use the baseline terminal condition approach which is used because we know the economy was in a form of state at that point. Therefore, for each country we considered the last value (e.g. 2007Q2) to correspond with the long-run steady state solution of the model and, hence, the baseline scenario should not be considered a projection. Solving the model produces a transitional (medium-run) steady state solution for the model variables, i.e. these variables will vary over time

\footnotetext{
${ }^{19}$ In principle, a more refined approaches could be utilized here, for instance direct estimation.

${ }^{20}$ The shocks considered are deterministic, but the model is estimated more in the stochastic framework therefore future research will explore the implications of stochastic simulations.

${ }^{21}$ There are no theorems that show that the extended Kalman filter will convergence to the true nonlinear filter even in a first-order solution. Indeed, as Atolia et al (2010)) show, the further an economy is from its steady-state equilibrium, the larger are the errors generated by linearization, both qualitatively and quantitatively.

${ }^{22}$ There have been many recent advances in stochastic solutions methods - see e.g. Aruoba et al (2006), Anderson (2010), and Kollmann et al (2010).
} 
due to the fact that movements from one point to another are not instantaneous. Furthermore, our approach is motivated by the understanding that the effects of the terminal condition on the solution at the beginning of the simulation fade out, if the solution period is lengthened far enough in the future (Roeger and in't Veld (1997)). To ensure this, we run the simulations over a long horizon - 300 years. Given recent technological advances, the computational time is small, and tests on this model show that there is effectively no impact of increasing this time-horizon. By simulating over such a long horizon we are able to ensure the convergence of the model solution back to its baseline steady state (temporary shocks) or to a new long-run steady state (permanent shocks) well before the end of the simulation period. Indeed, the stability properties of the NMCM are good reflected by the fact that the model converges to a steady-state typically within around 40 years. As we ignore the solution after 80 years, then little is lost by our choice of terminal conditions even in cases where the new steady state deviates from the baseline steady-state. ${ }^{23}$ Indeed, one of the key aspects of the model, is that long-run relationship between variables exist, and are explicitly modelled and that we allow feed-back from the short-run to the steady-state. ${ }^{24}$ However, further testing of alternative terminal conditions needs to be performed to study the impact on the steady-state.

This forward-looking approach addresses the Lucas critique, but probably does it in an unrealistically strict way. In this respect, we leave to our sister paper (Dieppe, Gonzalez Pandiella, Hall and Willman 2011) a more detailed analysis of alternative model-consistent expectation formation by exploring the implication of replacing rational expectations with learning and studying the impact of changes in the structure of the economy, e.g. changes in agents beliefs and changes in various structural parameters. In the sister paper we are also able to relax the assumption in the simulations below that all agents (household, firms, unions, central banks, and governments) have the same information set.

\section{Indicative Simulations}

We have highlighted the important role of economic theory in identifying the distinction between short-run and long-run effects in a model. However it is key to see how the whole system works and to assess the differences across countries. Given the homogeneous theoretical specification, we would expect that the trajectories of the shocks should be similar across the countries, however differences will arise from different parameter estimates (reflecting different structural or economic factors), different initial states of the economy, and from different adjustment speeds. This combines into complex dynamic structures. However, by looking at the roots of the equations (i.e. the degree of forward/backward lookingness) as well as the adjustments of the economy to fundamentals, we are able to provide a framework to interpret the simulations. For example, if a country is more forward-looking, then typically this implies quicker adjustment, whereas backward-looking behaviour reflecting frictions implies that shock effects are more persistent, i.e. distributed over a longer period and potentially leads to hump shaped adjustment paths. In this section we undertake a range of simulations to highlight the properties of the model for scenario and policy analysis. These simulations are not intended to provide a comprehensive account of the model's multipliers but are only indicative. Indeed, the model could be used to analyse many types of structural shocks, or even direct shocks to expectations.

The model can be run under alternative assumptions concerning response of interest rates, exchange rates, budgetary policy etc. In this paper we focus on the Autarky (single country) case, where the rest of the world is exogenous, namely: world demand and prices, world interest rates, and

\footnotetext{
${ }^{23}$ Given that we ignore the simulations after 80 years, we fix the interest rate 100 years ahead.

${ }^{24}$ Many DSGE model are done as deviations from some ad hoc trend. A mis-specified trend will generate bias in estimation (Cogley (2001), Gorodnichenko and Ng (2010)).
} 
oil prices ${ }^{25}$. Futhermore there are no cross-country linkages and monetary policy and exchange rates react to country developments. The fiscal rule (107) is in action but government consumption and investment follow in real terms exogenously determined paths, and other government expenditures remain constant as a share of GDP.

In this section we consider a variety of shocks. The first is an interest rate shock which informs us about the transmission of monetary policy within the NMCM. We then consider a permanent demand side shock, namely a permanent increase in government spending. Finally, we consider a permanent supply side shock - a technology shock - both labour or capital augmenting. These shocks thus explore temporary, permanent demand and permanent supply shocks. In addition we report an exchange rate and a world demand shock. In all cases the shocks and simulations start in 2010q1.

We start by considering an unanticipated shock starting at time $t$. As forward-looking agents don't anticipate the initial shock, there is no reactions before the shock materialises, but under the assumption of full-information, perfect foresight, and given the announcement is fully credible, rational agents are able to fully anticipate the subsequent behaviour of the economy, and future expectations are fully aligned with the outcome (i.e. fully model consistent). We assume that all sectors have the same information set in forming expectations. Although, this assumption of perfect foresight is extreme, these simulations will be a benchmark for simulations with alternative expectation assumptions and enable us to isolate the implication of relaxing this assumption.

We consider 5 shocks occurring at 2010q1:

1. Short-term interest rate shock - defined as a 50 b.p. increase in the short-term interest rate for 1 period followed by an interest rate rule.

2. Permanent Government expenditure shock - specified as a $0.5 \%$ of GDP increase in public consumption over the entire simulation horizon.

3. Technology (TFP) shock such that potential output is up by $0.1 \%$ over the entire horizon.

4. Permanent appreciation of the euro nominal exchange rate by $5 \%$ against all foreign currencies

5. Permanent World demand shock of $1 \%$ increase in extra euro area import demand.

The simulations are presented in the figures at the end of the paper, where the results are presented as deviations from baseline, (real variables and employment are expressed as percentages deviations; prices as differences in year-on-year inflation rates and for interest rates, savings ratio, fiscal deficit, and unemployment, they are expressed as absolute deviations, either in percentage points or percent of GDP). We present results for the 5 estimated countries and consider the mechanisms and implications for the 5 shocks in turn.

\subsection{Short-term interest rate shock}

This simulation is where the central bank announces a one-off increase in the short-term interest rate of 50 b.p. for 1 period, and thereafter will revert to previous behaviour - i.e. interest rates will subsequently follow the Taylor rule.

The transmission of monetary policy operates through a number of channels. First, via a change in real exchanges rates - due to the real UIP, which impacts both import prices and competitiveness. Initially the exchange rate appreciates to make possible future depreciation in anticipation of future decreases in the interest rate. This exchange rate effect damages competitiveness and, therefore,

\footnotetext{
${ }^{25}$ Endogenisation of these variables would require an global model.
} 
the trade balance. Secondly, monetary policy affects the user-cost of capital, whereby, long-term interest rates follow the term structure - and hence the average of future short-term interest rates. This raises the user cost of productive and residential capital, thereby acting as a depressant on firm and housing investment. Thirdly, households are directly affected due to higher discount rate, and by intertemporal substitution effects where savings and future consumption become more attractive. Another channel is that net interest payments are also affected for firms, households and governments. Finally, expectations play a key role in all of this; for example households are already in the first period affected by expected lower wage growth which immediately decreases their consumption expenditure.

The combined effect of all this is a reduction in final demand as firms reduce output and thus, the demand for factors of production. This depresses prices, and combined with lower employment creation, causes lower wages and lower household income. The lower demand decreases imports, alleviating somewhat the downward pressures on GDP. As capital becomes relatively more expensive than labour and as labour has a smaller adjustment cost than capital then firms reduce employment. Interest rates only gradually adjust down following a one period shock due to interest rate smoothing. Therefore, the price effect is relatively large as agents realise that the shock to interest rates takes time to unwind. However, lower prices starts to lead to gains in competitiveness, which combined with improvements in productivity results in output growth returning to its previous level, halting the decrease in inflation. Overall, a temporary shock to short-term interest rate leads to a temporary fall in real GDP but a more gradual decrease in the price level. After 5 years, interest rates are nearly back to base.

The differences across countries are due to the different structures of their economies, and reflect the different estimated parameters, e.g. the speed of adjustments of factor demands and the extent to which agents are forward-looking. Indeed, there are some notable differences, both in terms of timing, and especially, the size of the interest rate shock impact across the countries. On the real side, the largest effect is for Spain and Italy reflecting partly stronger real interest rate effects and high interest rate sensitivity of investment, but also reflecting that firms investment decisions are estimated to be more forward-looking than in other countries. For all countries there is also significant habit persistence in households. In France consumption is least dependent on current income and wealth, instead, on long lags and leads of income formation. The timing and size of the inflation reaction also differs across countries. These can be explained by the specific estimation results. On the price side, the more flexible prices are, that is, the larger the part of price setters who change prices in a given period, the larger is the inflation response. Indeed, the speed of price adjustment towards its equilibrium also seems to explain the relative price responses. Spain changes their prices the most often, (on average every 3 quarters) implying a quicker inflation response. Wage behaviour also plays a key role, where, for instance, in Spain we find high indexation of nominal wages to prices.

The introduction of model-consistent, forward-looking behaviour leads to quick initial reactions even though the model contains quite a number of rigidities. This is in slight contrast to the studies conducted under the ESCB's Monetary Transmission Network (MTN) ${ }^{26}$ or DSGE models e.g. Smets and Wouters (2003), or Christoffel et al (2008), which suggest that the maximum decline in output is reached after about four quarters. As we see in our sister paper, changing the expectations to learning add some additional delay to the output responses. However, as with VAR models of Euro area monetary transmission, e.g. Peersman and Smets (2001), or DSGEs (Smets and Wouters 2003 or Christoffel et al 2008), we find a relatively large investment reaction across countries, which is between 2 to 3 times larger than the GDP response, whereas consumption responds less. The MTN findings suggest that transmission mechanisms in the individual countries are similar in broad terms (as we also find), but the evidence from the literature on cross-country responses to a monetary shock show

${ }^{26}$ See Angeloni et al (2003) for a summary. 
large, policy-independent, heterogeneity (Angeloni and Ehrmann, 2003).

Van Els et al (2001) did a comparative analysis based on euro area macroeconometric models. Although their simulation design was different, the relative size of maximum output losses in response to a monetary policy shock was the same as in the NMCM, with Netherlands and France having the smallest output losses, and Italy and Spain having the largest, but this finding is different to some SVAR analysis (e.g. Clements et al (2001) and Ehrmann (1998)).

\subsection{Permanent Government expenditure shock}

This can be considered as an unanticipated but credible announcement of an immediate and permanent increase in government spending of $0.5 \%$ of GDP.

The increase in demand leads directly to an increase in employment. Firms initially increase their investment, reflecting a positive accelerator effect. The non-Ricardian households also observe the increase in their current income, however, the increase in government spending leads to a less favourable government financial position which needs to be financed. This is only partially done by a reduction in government transfers to households because of lower unemployment. The fiscal rule employed in the model, will mean households will be affected by higher tax rates in order to bring the Government debt as a share of GDP back to normal. Therefore, households will have less future disposable income and will consequently reduce their current consumption. Employees will also pass on part of the tax burden to employers by negotiating higher wages. This will, to some extent, mitigate the tax effects. On impact, GDP increase by only around $0.5 \%$.

This stronger demand puts upward pressure on prices and wages. The main transmission from a positive demand shocks to prices is via marginal costs directly affecting the hybrid new Keynesian price and wage Phillips curves and so unions respond to declining unemployment by increasing their wage demands and firms respond by increasing prices. Further pressure on wages is due to the increase in tax rates which aim to ensure sustainability of government finances. Through the increase in inflation, domestic producers become less competitive, so exports tend to decrease and imports increase, leading to a worsening trade balance. Furthermore, the higher marginal costs, and higher inflation, drives up the interest rates which subsequently has a negative impact on investment and puts further downward pressure on consumption. There is also a persistent increase in domestic price level that implies that part of increased government consumption is absorbed by lower exports. After around 5 years of increased government spending, GDP and inflation return to baseline levels.

The main differences across countries come from different estimated speeds of adjustment of wages and prices as well as different frictions associated with adjustment to factor demands by firms, and the behaviour of unions and households. Price and wage dynamics are essential to explaining these differences. According to these simulation, France and Netherlands show the quickest price responses.

\subsection{Technology shock}

In addition to the demand shock we consider a supply shock in the form of permanent technology shocks. As we have a CES production function we consider a shock both to labour augmenting and capital augmenting technical progress, such that in both cases the (ceteris paribus) effect on potential output is $0.1 \%$ - i.e. with the baseline amounts of capital and labour the economy could produce $0.1 \%$ more output.

Broadly taken the effects of both technology shocks are very similar on most variables. Impact and short-run effects on GDP are below $0.1 \%$ in all countries except in Spain, where the impact effect is just above 0.1, however, thereafter falling below that level. The incomplete short-run transmission 
of the shocks to output reflects negative short-run labour demand responses to both shocks, i.e. positive supply effects are partly crowded out. In the technology shock literature negative employment responses to technology shocks are quite generally interpreted as a new Keynesian feature reflecting price stickiness. Cantore et al. (2010), however, showed that from the point of view of this result costly adjustment of investment played a markedly more crucial role. They also showed that, although the impact response employment to a capital augmented shock was positive with close enough to zero values of substitution elasticity between capital and labour, this impact could be negative well below unity value of substitution elasticity when coupled with positive adjustment costs of investment. That is also what our simulation results depict. Hence, short-run employment responses to both capital and labour augmenting shocks are negative and differences in the effects of these two technology shocks are more quantitative than qualitative. The labour augmenting shock result in larger negative employment and positive investment effects than the capital augmenting shock. Positive real wage and consumption effects, in turn, are larger in response to the capital augmenting shock. Hence, as the well known implication of the CES production function with labour augmenting technical progress is the constancy of equilibrium factor income shares, the higher real wage and the lower capital stock response to the capital augmenting shock than to the labour augmenting shock in our simulations imply that in the long run the capital augmenting shock raises the equilibrium labour income share. This is fully in line with the discussion, for example, by Acemoglu (2009) that capital augmenting technical progress favours labour income (capital income), if the elasticity of substitution is below (above) unity.

\subsection{Permanent Exchange rate shock}

This is a permanent appreciation of the euro nominal exchange rate by $5 \%$ against all foreign currencies.

In the short-run, the main transmission channel is due to a decrease in competitiveness, leading to lower exports to countries outside the euro area but also higher imports. However the weaker demand for domestically produced goods and lower import prices has a deflationary price effect leading to lower interest rates. With some wage rigidities, this has the consequence of boosting real consumption due to higher income. Gradually the lower prices erode the competitiveness losses, and combined with a more relaxed monetary policy, the economies gradually return to their baseline levels of output and inflation. However, as the shock is a nominal shock, it has no long-run effects on the real variables, but prices settle down to permanently lower levels.

In the short-run, the cross-country differences mainly reflect the sensitivity of net exports to changes in competitiveness and in particular the speed of the exchange rate pass-through to competitors prices.

\subsection{Permanent World demand shock}

The last diagnostic simulation is a permanent foreign demand shock which is a $1 \%$ increase in extra euro area import demand.

Higher foreign demand directly increases exports. There are cross-country differences which mainly depend on the exposure of the countries to the rest of the world, with Germany and Netherlands having the largest initial positive GDP effects due to a large export trade market. Higher demand also boosts imports, which is conditional on the import context of exports. Higher demand also boosts employment, lowering unemployment which increases wage demands and production prices leading to inflationary pressures and hence monetary tightening. One would expect other changes in the 
international environment, e.g. foreign interest rate changes, but in this scenario we assume that all other foreign variables are unchanged, which perhaps is not so realistic.

\subsection{Pre-announced (anticipated) shocks}

In the previous section we considered an unexpected and unanticipated (not pre-announced) events hitting the economy. As the initial shock was not pre-announced at time t, there is no reactions before the shock materialises. However at time $\mathrm{t}$ the future path is announced, and providing the announcement is credible then under rational expectations, forward-looking agents can anticipate the consequences right from the start of the shock, i.e. agents know the future interest rate and government expenditure paths. However, in this model framework, we can also consider pre-announced economic events, for example a change in government policy to occur in the future. We can then consider both the current period impacts of pre-announced changes - i.e. the impact of anticipated events or changes in policy as well as the subsequent dynamic path. In this case we consider the pre-announcement to be credible, i.e. agents fully expect the action to take place.

To illustrate the difference between an pre-announced and shock we consider a temporary increase in government spending of $0.5 \%$ of GDP for 3 years under the two alternative conditions:

- pre-announced at time $t$ - 8 . In this case the change in government policy is pre-announced 2 years before the shock and is fully credible and anticipated. Therefore, forward-looking agents know that the shock will occur and can already optimise their behaviour in advance.

- announced shock occurring at time $t$, This is similar to the simulations in the previous section but is only a temporary shock.

In the unanticipated shock there is a jump in prices when the shock takes place as agents re-adjust their expectations. In the 'anticipated/pre-announced' case agents can react in advance, as they know, not only the timing, but also the duration and size of the shock. We see that initially prices react less, as some agents are backward looking. However, interest rates, and exchange rates already start to react in advance leading to an initial negative effect on GDP.

\subsection{Unannounced (unanticipated) change in policy}

In the previous simulations, forward-looking agents anticipate fully the consequences of a shock right from the start of the announcement, however, this could be viewed as assuming too much information on behalf of agents. Therefore, we also consider an unannounced and unanticipated changes in policy, in which agents are continuously surprised. Agents in this case observe the shock, but expect it to be reversed the next period. This could be due to limited information by agents or because the change in policy is unannounced or is considered 'un-credible' by agents. In a way, this approach is consistent with the DSGE practice where shocked variables are expected to follow an estimated AR structure. However, forward-looking agents are still rational, and expectations are still modelconsistent it is just that agents don't fully know the full sequence of future shocks and are to some degree, continuously (sequentially) surprised each period. This approach differs from the previous simulations, in that agents will have to continuously re-optimize his or her behaviour taking into account the new information, whereas in the previous cases, the outcome is in line with the optimising behaviour of forward-looking agents. Clearly, for a one period shock the announced and unannounced shocks are the same, but divergences occur when the shock lasts over a longer period of time. 
We consider a three year increase in Government spending of $0.5 \%$ of GDP for three years and thereafter government spending returns to the baseline. The fiscal rule is in operation and known by agents. We consider this scenario under three alternative assumptions:

1. announced and credible shock, where at time t the government announces the increase in government spending and the duration. This announcement is credible, so agents adjust their expectations accordingly;

2. an unannounced (or uncredible) and unanticipated shock to government spending where agents don't know that government policy has changed so they expect government spending to return to its previous level each period and

3. an unannounced (or uncredible) and unanticipated shock, where forward-looking agents expect a Government spending shock to display some persistence but gradually fading out - i.e. agents assume government spending follows an $\mathrm{AR}(1)$ process with a rho parameter of 0.9 .

In each case the shock is for 3 years, and expectations are rational in that they are model-consistent. The only difference is whether the agents realise the duration of the shock or not. In the first case they know the duration as it is announced by the government. In the second and third simulation, the duration of the shock is unknown so agents make alternative assumptions about the duration. In these cases the model is solved iteratively as agents re-optimise each period as new information becomes available.

For simplicity we have done these simulations only for France, and the results are presented at the end of the paper. Qualitatively, the three simulations have similar reactions: output increases, prices rise, and interest rates increase due to higher demand and higher prices; but there are quantitative differences both in magnitude and timing. Under the announced shock, the initial impact on GDP is less than the size of the initial shock, as agents anticipate future tax rises. However, under the unannounced shocks, the initial impact of GDP is around 0.5\%. Further out the negative impact of GDP is larger in the unannounced cases compared to the announced shock. On the price size, the initial effects for the unannounced shocks are small, but quickly increase, with the unannounced AR(1) expectations having the largest impact on prices. Therefore, the reaction of the economy to identical paths of government spending depends strongly on agents expectation of the duration of the shock. Under the assumption of a gradual return to previous levels, the rigidities of the model and the distinction between forward-looking and backward agents becomes apparent. After 20 years all three simulations are back to base. We explore this issue further in our sister paper (Dieppe et al 2011) where we consider the implication of replacing rational expectations with learning.

\section{Conclusions}

In this paper, we have presented a model specified to track both the short-run dynamics around the medium-run equilibrium and also the deviations of the medium-run developments from the balancedgrowth-path. The model consists of three optimising private sector decision making units, i.e. firms, trade unions and households, where output is in the short-run demand determined and monopolistically competing firms set prices and factor demands. Labour is indivisible and monopoly-unions set wages and households make consumption/saving decisions. In the medium-run the supply-side has cross-equation restrictions which allows for non-unitary elasticity of substitution between labour and capital and quite general factor augmenting technical progress. The micro-founded theoretical coherance of the model addresses the Lucas critique, even though we assume agents knows only the 
parameters related to her optimization problem but does not need to know the rest of the model, i.e. a limited information approach. Therefore, the core of the model is estimated with GMM, which implicitly assumes limited information boundedly rational expectations.

In this paper, indicative simulations are undertaken with agents' expectations based on rational, model consistent expectations. These simulations suggest the impluse responses of the model to exogenous shocks are plausible with cross-country differences reflecting estimated country heterogeneity. Furthermore, under our framework, the reaction of the economy to shocks depends strongly on whether it is pre-announced and anticipated or announced and credible, or unannounced and uncredible.

Previous criticism of backward looking macroeconometric models, have been that real effects are too long-lasting. In the new MCM with forward-looking rational expectations, even with some rigidities, the initial adjustments are quick and there are fast adjustments to equilibrium. This is comparable to many DSGE models, but to some degree the adjustment could be too quick, unless agents do not know the duration of the shocks, e.g. they suppose it is a series of autocorrelated exogenous shocks. This is one motivation for considering an alternative expectation process. Indeed, the rationality assumption used in these scenarios are in some ways extreme: either there is full credibility or zero credibility and agents are continuously surprised. An alternative, more plausible case is that credibility is acquired. In a subsequent paper we approach this issue by introducing some learning mechanisms into the model in our sister paper (Dieppe, Gonzalez Pandiella, Hall and Willman 2011) and study the impact. In future papers we shall also conduct various simulation exercises to study the cross-country linkages.

\section{References}

Acemoglu, D. (2002), 'Directed Technical Change,' Review of Economic Studies, vol. 69(4), pages 781-809, October.

Acemoglu, D. (2003), 'Labor- And Capital-Augmenting Technical Change,' Journal of the European Economic Association, vol. 1(1), pages 1-37, 03.

Acemoglu, D. (2009), 'Introduction to Modern Economic Growth', MIT Press

Akerlof, G. A. (2002), 'Behavioral Macroeconomics and Macroeconomic Behaviour', American Economic Review, 92(3), 411-433.

Akerlof, G. A. and J. L. Yellen, (1985), 'Can Small Deviations from Rationality Make Significant Differences to Economic Equilibria?', American Economic Review, 75(4), 708-720.

Altissimo, F., M. Ehrmann, and F. Smets, (2006), 'Inflation persistence and price-setting behaviour in the euro area - a summary of the IPN evidence,' Occasional Paper Series 46, European Central Bank.

Álvarez, L. J., E. Dhyne, M. Hoeberichts, C. Kwapil, H. Le Bihan, P. Lünnemann, F. Martins, R. Sabbatini, H. Stahl, P. Vermeulen, and Jouko Vilmunen, (2006), 'Sticky Prices in the Euro Area: A Summary of New Micro-Evidence', Journal of the European Economic Association, vol. 4(2-3), pages 575-584, 04-05.

Anderson, G.S, (2010), 'A reliable and computationally efficient algorithm for imposing the saddle point property in dynamic models', Journal of Economic Dynamics and Control, doi:10.1016/j.jedc.2009.10.004. Angeloni, I., and M. Ehrmann, (2003), 'Monetary transmission in the euro area: early evidence', Economic Policy, 37, 470-492.

Angeloni, I ., Kashyap, A. and B. Mojon (2003), 'Monetary Policy Transmission in the Euro Area. A Study by the Eurosystem Monetary Transmission Network', Cambridge University Press, 514 pp., ISBN: 0521828643 
Aruoba S. B, Fernández-Villaverde J, Rubio-Ramírez J (2006), 'Comparing solution methods for dynamic equilibrium economies', Journal of Economic Dynamic and Control 30, 2477-2508

Atolia, M, S Chatterjee, and S. Turnovsky, (2010), 'How misleading is linearization? Evaluating the dynamics of the neoclassical growth model', Journal of Economic Dynamics and Control 34, no. 9, 1550-1571.

Beeby, M., Hall, S.G. and S.G. Brian Henry, (2004), 'Modelling the Euro-11 Economy: A Supply-Side Approach', in S.G. Hall, U. Heilemann and P. Pauly (eds), Macroeconometric Models and European Monetary Union, Duncker\&Humblot, Berlin, 19-40.

Bils, M. (1987), 'The Cyclical Behavior of Marginal Cost and Price', American Economic Review, 77, $838-855$

Blanchard, O. J. (1985), 'Debt, Deficits, and Finite Horizons', Journal of Political Economy, vol.93(2), 223-247.

Blanchard, O. J. (1997), 'The Medium Run', Brookings Papers on Economic Activity, 1997-2, 89-158. Boissay, F. and J.P. Villetelle, (2005), 'The French block of the ESCB Multi-Country Model', ECB Working Paper, No. 456

Box, G. and D. Cox (1964), 'An Analysis of Transformations', Journal of the Royal Statistical Society, 26, Series B, 211-243.

Bray M.M.(1983), 'Convergence to Rational Expectations Equilibrium' in R Frydman and E.S. Phelps (eds) 'Industrial Forecasting and Aggregate Outcomes' Cambridge University Press, Cambridge.

Cantore, C., M. A. León-Ledesma, P. McAdam and A. Willman, (2010), 'Shocking Stuff: Technology, Hours, and Factor Substitution', ECB Working Paper No. 1278.

Christoffel K, Coenen G, Warne A, (2008), 'The new area-wide Model of the Euro Area: a microfounded open-economy model for forecasting and policy analysis.' ECB Working Paper 944

Clarida, R., Gali, J. and M. Gertler (2000), 'Monetary policy rules and macroeconomic stability: evidence and some theory'. Quarterly Journal of Economics, February 2000, pp. 147-180.

Clements, B., Z. G. Kontolemis and J. Levy, (2001), 'Monetary policy Under EMU: Differences in the Transmission Mechanism?' IMF Working Paper, WP/01/102, International Monetary Fund, Washington, D.C.

Cogley, T., (2001), 'Estimating and testing rational expectations models when the trend specification is uncertain', Journal of Economic Dynamics and Control 25(10), 1485-1525.

Constanides, G.M., (1990), 'Habit Formation: A Resolution of the Equity Premium Puzzle', Journal of Political Economy; 98(3), June 1990, 519-43.

De Walque, G., O. Pierrard, H. Sneessens and R. Wouters (2009). 'Sequential Bargaining in a NewKeynesian Model with Frictional Unemployment and Staggered Wage Negotiation", ECB Working Paper No. 1007.

Deaton, A. and J. Muellbauer (1980), 'An Almost Ideal Demand System', American Economic Review, Vol 113, 253-276.

Dias, D., Dossche, M., Gautier, E., Hernando, I., Sabbatini, R., Stahl , H. and Vermeulen, P., (2007), 'Macro Price setting in the euro area: Some stylised facts from Individual Producer Price,' Documents de Travail 164, Banque de France.

Dieppe A., A. Gonzalez Pandiella, S. Hall and A. Willman (2011), 'The ECB's New Multi-Country Model for the Euro area: NMCM - with Boundedly Rational Learning Expectations' ECB Working paper No. 1316.

Druant, M., S. Fabiani, G. Kezdi, A. Lamo, F. Martins and R. Sabbatini (2009), 'How Are Firms' Wages and Prices Linked: Survey Evidence in Europe', ECB Working Paper No. 1084.

European Central Bank (2001), 'A Guide to Eurosystem Staff Macroeconomic Projection Exercises', European Central Bank, June 2001. 
Eichenbaum, M., Nir Jaimovich and S. Rebelo (2008), 'Reference Prices and Nominal Rigidities', NBER Working Papers 13829, National Bureau of Economic Research, Inc.

Evans G.W (1986), 'Selection Criteria for models with non-uniqueness' Journal of Monetary Economics, 12, 147-157.

Evans G.W.(1989), 'The fragility of sunspots and bubbles' Journal of Monetary Economics, 23, 297317

Fabiani, S., M. Druant, I. Hernando, C. Kwapil, B. Landau, C. Loupias, F. Martins, T. Mathä, R. Sabbatini, H. Stahl and Ad Stokman, (2006), 'What Firms' Surveys Tell Us about Price-Setting Behavior in the Euro Area,' International Journal of Central Banking, vol. 2(3), September.

Fagan, G., and J. Morgan, (2005), 'An overview of the structural econometric models of the euroarea central banks', in G. Fagan and J. Morgan (eds), Econometric Models of the Euro-Area Central Banks, Edward Elgar Publishing, Chapter 1, 1-49.

Friedman B. (1975), 'Rational Expectations are really Adaptive after all' discussion paper No.430 Howard institute of economic research.

Gabaix, X. and D. Laibson (2001), 'The 6D Bias and the Equity-Premium Puzzle', NBER Macroeconomics Annual, 257-312.

Gali, J. (1990), 'Finite horizons, life-cycle savings, and time-series evidence on consumption', Journal of Monetary Economics 26, 433-452.

Gali, J. and M. Gertler (1999), 'Inflation Dynamics: A Structural Econometric Analysis', Journal of Monetary Economics, 44, 195-222.

Galí, J and M. Gertler and David López-Salido (2001), 'European inflation dynamics,' European Economic Review, vol. 45(7), pages 1237-1270.

Gorodnichenko, Y. and Ng, S. (2010), 'Estimation of DSGE models when the data are persistent', Journal of Monetary Economics, vol. 57(3), pages 325-340.

Hansen, L. P. (1982), 'Large sample properties of generalized method of moments estimators', Econometrica 50, 1029-1054.

Hansen, G.D. (1985), 'Indivisible Labor and the Business Cycle', Journal of Monetary Economics, 16, 309-327.

Hubrich, K and T. Karlsson (2010), 'Trade Consistency in the Context of the ESCB Projection Exercises - An overview', ECB Occassional paper, 108.

Juillard, M., D. Laxton, P. McAdam, and H. Pioro, (1998), 'An algorithm competition: First-order iterations versus Newton-based techniques,' Journal of Economic Dynamics and Control, vol. 22(8-9), pages 1291-1318, August.

Kinoshita, T. (1987), 'Working Hours and Hedonic Wages in the Market Equilibrium', Journal of Political Economy, 95, 6, 1262-77

Klump, R., P. McAdam and A. Willman (2007), 'Factor Substitution and Factor Augmenting Technical Progress in the US', Review of Economics and Statistics, 89, 1, 183-192.

Klump R, McAdam P and Willman A (2007), 'The long-term sucCESs of the neoclassical growth model', Oxford Review of Economic Policy, 23(1):94-114

Klump, R., P. McAdam and A. Willman (2008), 'Unwrapping some euro area growth puzzles: Factor substitution, productivity and unemployment', Journal of Macroeconomics, 30, 645-666.

Kollmann,R, S Maliar,B.Malinand and P Pichler, (2010), 'Comparison of solutions to the multicountry real business cycle model', Journal of Economic Dynamics \& Control, doi:10.1016/j.jedc.2010.09.013 La Grandville, O. de (1989), 'In quest of the Slutzky diamond', American Economic Review, 79, 468-481.

León-Ledesma, M., P. McAdam and A. Willman (2010), 'Identification of the elasticity of substitution with biased technical change', American Economic Review, 100, 1330 -1357. 
Maćkowiak, B and M. Wiederholt, (2010), 'Business Cycle Dynamics Under Rational Inattention', CEPR Discussion Paper No. DP7691.

Markowitz, H. (1952), 'Portfolio Selection', The Journal of Finance, 7(1), 77-91.

Marron, J.S. and M. P. Wand, (1992), 'Exact Mean Integrated Squared Error', Annals of Statistics, 20(2), 712-736.

McAdam, P. and A. Willman (2004), 'Supply, Factor Shares and Inflation Persistence: Re-Examining Euro-Area New-Keynesian Phillips Curve', Oxford Bulletin of Economics and Statistics, 66, 637-670. McAdam, P. and Willman A (2008), 'Medium run redux: Technical change, factor shares and frictions in the euro area', ECB Working Paper No. 915.

McAdam P and Willman A (2010), 'State-Dependency and Firm-Level Optimization: A contribution to Calvo Price Staggering' Manchester School, 78, 556-581.

Muellbauer, J. (1988), 'Habits, Rationality and Myopia in the Life Cycle Consumption Function', Annales D'Economie et Statistique, no. 9, 47-70.

Muellbauer, J. and R. Lattimore (1995), 'The Consumption function: A Theoretical and Empirical Overview', In J. H. Pesaran and M. Wickens (eds.), Handbook of Applied Econometrics, Blackwell Handbooks in Economics, 221-311.

Peersman, G. and F. Smets, (2001), 'The monetary transmission mechanism in the Euro area: more evidence from VAR analysis (MTN conference paper),' ECB Working Paper Series No. 091.

Radner R. (1982), 'Equilibrium under Uncertainty' in K.J.Arrow and M.D.Intriligator, (eds) Handbook of Mathematical Economics, vol 2, North Holland, Amsterdam

Reis, R. (2006), 'Inattentive consumers', Journal of Monetary Economics, 1761-1800.

Roeger,W. and J. in 't Veld (1997), 'Simulating Permanent Shocks with QUEST II: Sensitivity of Results to Terminal conditions', Paper presented at TROLL 1997 Conference, Brussels.

Rogerson, R. (1988), 'Indivisible labor, lotteries and equilibrium', Journal of Monetary Economics, 21, 3-16.

Rotenberg, J.J. (1987), 'New Keynesian Microfoundations', In S. Fischer (ed.), NBER Macroeconomics Annual, Cambridge, StateMA: MIT Press, 69-104.

Schmitt-Grohé, S. and M. Uribe (2003), 'Closing small open economy models', Journal of International Economics, 61, 163-185

Shapiro, M. D. (1996), 'Macroeconomic Implications of Variation in the Workweek of Capital ', Brookings Papers on Economic Activity, vol. 27, issue 1996-2, pages 79-134

Sims, C. A.. (2003), 'Implications of Rational Inattention', Journal of Monetary Economics, 50(30), 665-690.

Skinner, J. (1988), 'Risky Income, Life Cycle Consumption, and Precautionary Savings', Journal of Monetary Economics, 22, 237-255.

Smets F. and R. Wouters, (2003), 'An Estimated Dynamic Stochastic General Equilibrium Model of the Euro Area,' Journal of the European Economic Association, vol. 1(5), pages 1123-1175, 09

Solow, R. M. (2000), 'Toward a Macroeconomics of the Medium Run', Journal of Economic Perspectives, American Economic Association, vol. 14(1), pages 151-158, Winter.

Sundaresan, S. (1989), 'Intertemporally Dependent Preferences and the Volatility of Consumption and Wealth', Review of Financial Studies; 2(1), 1989, 73-89.

Taylor, J. B. (1999), 'Staggered price and wage setting in macroeconomics', In: J.B. Taylor and M. Woodford, Editors, Handbook of Macroeconomics Vol. 1, North-Holland, Amsterdam (1999), pp. 1009-1050.

Thaler, R. H. (1994), 'Psychology and Saving Policies', American Economic Review, Papers and Proceedings, 84(2), 186-192. 
Thaler, R. H. (2000), 'From Homo Economicus to Homo Sapiens', Journal of Economic Perspectives, 14(1), 133-141.

Townend R.M. (1978), 'Market anticipation, rational expectations and Bayesian analysis', International Economic review, 19, 481-494.

Townend R.M. (1983), 'Forecasting the forecasts of others', Journal of Political Economy, 91,546-588 Trejo, S. J. (1991), 'The Effects of Overtime Pay Regulation on Worker Compensation', American Economic Review, 81, 4, 719-740.

Van Els, P., A. Locarno, J. Morgan and J. P. Villetelle, (2001), 'Monetary Policy Transmission in the Euro Area: What Do Aggregate and National Structural Models Tell Us?', ECB Working Paper, No. 94.

Wage Dynamic Network (2010), 'Wage Dynamics in Europe Final Report of the Wage Dynamics Network' see: http://www.ecb.europa.eu/home/html/researcher_wdn.en.html

Willman, A. (2002), 'Estimation of The Area-Wide Production Function And Potential Output', ECB Working Papers No. 153.

Willman A (2007), 'Sequential Optimization, Front-Loaded Information and U.S. Consumption', ECB Working Papers No. 765.

Willman, A. and A. Estrada (2002), "The Spanish block of the ESCB-multi-country model" ECB Working Papers No. 149.

Willman, A., M. Kortelainen, A. Männistö and M. Tujula (2000), 'The BOF5 macroeconomic model of Finland', Economic Modeling, 17, 2, 275 - 303.

Woodford, M. (2003), 'Interest and Prices: Foundations of a Theory of Monetary Policy', Princeton University Press.

Zeldes, S. P. (1989), 'Optimal consumption with stochastic income: Deviations from certainty equivalence', Quarterly Journal of Economics, CIV(2), 275-298. 


\section{A Appendix: Instruments and normalisation for some key equations.}

\section{A.1 Employment demand}

\begin{tabular}{|r|r|r|r|r|r|}
\hline & \multicolumn{5}{|c|}{ Lags of Instruments } \\
\hline Instruments & $\mathrm{DE}$ & $\mathrm{FR}$ & $\mathrm{IT}$ & $\mathrm{ES}$ & $\mathrm{NL}$ \\
\hline$\Delta \mathrm{n}$ & $2-4$ & $1-4$ & 2 & 3 & 2,3 \\
\hline$\Delta \mathrm{y}$ & 1,3 & $1-3$ & 4 & 2 & $2-4$ \\
\hline $\mathrm{n}^{*}-\mathrm{n}$ & 2,3 & $1-3$ & 3,4 & $2-4$ & $2,3,5$ \\
\hline$\frac{(1+\mu)\left(w-p_{I}\right)}{F_{N}}$ & $1,3-6$ & $1,2,5,6$ & $3,4,6$ & $3,4,6$ & $2,3,6$ \\
\hline$\Delta\left(\mathrm{w}-\mathrm{p}_{I}\right)$ & 2,3 & $1-3$ & $2-4$ & $2-4$ & $3-5,7$ \\
\hline
\end{tabular}

Replacing the time varying discount factor, D, replaced by its sample average gives a simplified representation:

FR: $n_{t}=0.4981 n_{t+1}+0.4968 n_{t-1}+0.0051 n_{t}^{*}$
DE: $n_{t}=0.4881 n_{t+1}+0.4927 n_{t-1}+0.0192 n_{t}^{*}$
IT: $n_{t}=0.4840 n_{t+1}+0.4887 n_{t-1}+0.0273 n_{t}^{*}$
ES: $n_{t}=0.4650 n_{t+1}+0.4688 n_{t-1}+0.0662 n_{t}^{*}$
NL: $n_{t}=0.4889 n_{t+1}+0.4916 n_{t-1}+0.0195 n_{t}^{*}$

\section{A.2 Investment}

\begin{tabular}{|r|c|c|c|c|c|}
\hline & \multicolumn{5}{|c|}{ Lags of Instruments } \\
\hline Instruments & $\mathrm{DE}$ & $\mathrm{FR}$ & $\mathrm{IT}$ & $\mathrm{ES}$ & $\mathrm{NL}$ \\
\hline$\Delta \mathrm{k}$ & 2,3 & 2,3 & 2 & 3 & 2 \\
\hline INST1 & 1 & 1,2 & - & - & - \\
\hline $\mathrm{INST} 2$ & 2 & 1 & 2 & 2,3 & $2-4$ \\
\hline $\mathrm{i}_{\text {short }}$ & $2-4$ & $1-4$ & $2-4$ & 2,4 & 1,4 \\
\hline $\mathrm{i}_{\text {long }}$ & $1-5$ & $1-5$ & $1-5$ & $1-5$ & $1,3,4,6$ \\
\hline$(1+\mathrm{r})^{-1}$ & 1 & 1,2 & 1 & 2,3 & 2,3 \\
\hline $\mathrm{UC}$ & 2,4 & $1,2,4,5$ & 2,4 & 2,3 & $2-4$ \\
\hline $\mathrm{MPK}$ & $1,2,4,6$ & $2,4,6$ & $1,2,4,6$ & $1,2,4,6$ & $1,2,4,6$ \\
\hline$\Delta \mathrm{p}_{S}$ & $1,3,4$ & 1,3 & $1,3,4$ & $1,3,4$ & 2 \\
\hline $\mathrm{p}_{s}-\mathrm{p}_{s}^{h p}$ & - & - & - & 2 & 2,4 \\
\hline$\Delta \mathrm{y}$ & 2 & 1 & 2 & 2 & $2-4$ \\
\hline$\Delta \mathrm{x}$ & 1,3 & 1,3 & 1,3 & 1,3 & 1,3 \\
\hline
\end{tabular}

Where INST1 and INST2 are the fits of $\Delta k_{t+1}$ and $\Delta k_{t+2}$ with respect to the lagged values of capital stock, output, GDP deflator, investment deflator, user cost, marginal product of capital and stock prices. Only lags the t-values of which exceeded one were approved.

Normalising the equation with respect to the current period and replacing the time-varying discount factor by its sample average gives the following representation:

$\mathrm{FR}: \Delta k_{t}=-0.1166 \cdot \Delta k_{t+2}+0.6181 \cdot \Delta k_{t+1}+0.3568 \cdot \Delta k_{t-1}+0.0101 \cdot\left[M P K_{t}-0.75 \cdot U C_{t}+0.0246\right]$
$\mathrm{DE}: \Delta k_{t}=-0.0911 \cdot \Delta k_{t+2}+0.5600 \cdot \Delta k_{t+1}+0.4551 \cdot \Delta k_{t-1}+0.0080 \cdot\left[M P K_{t}-0.74 \cdot U C_{t}+0.0329\right]$
$\mathrm{IT}: \Delta k_{t}=-0.1160 \cdot \Delta k_{t+2}+0.6181 \cdot \Delta k_{t+1}+0.4593 \cdot \Delta k_{t-1}+0.0076 \cdot\left[M P K_{t}-0.80 \cdot U C_{t}+0.0271\right]$ 
$\mathrm{ES}: \Delta k_{t}=-0.1476 \cdot \Delta k_{t+2}+0.6898 \cdot \Delta k_{t+1}+0.4310 \cdot \Delta k_{t-1}+0.0076 \cdot\left[M P K_{t}-0.75 \cdot U C_{t}+0.0206\right]$ $\mathrm{NL}: \Delta k_{t}=-0.0617 \cdot \Delta k_{t+2}+0.4739 \cdot \Delta k_{t+1}+0.4740 \cdot \Delta k_{t-1}+0.0119 \cdot\left[M P K_{t}-0.67 \cdot U C_{t}+0.0206\right]$

\section{A.3 NKPC equation}

\begin{tabular}{|r|c|c|c|c|c|}
\hline & \multicolumn{5}{|c|}{ Lags of Instruments } \\
\hline Instruments & $\mathrm{FR}$ & $\mathrm{IT}$ & $\mathrm{NL}$ & $\mathrm{DE}$ & $\mathrm{ES}$ \\
\hline$\Delta \mathrm{p}$ & 2 & $1-3$ & 2 & 2 & 2,3 \\
\hline $\mathrm{p}^{*}-\mathrm{p}$ & 2,3 & 1,2 & 2,3 & - & - \\
\hline INSTPV & - & - & - & $1,2,3$ & 1,2 \\
\hline $\mathrm{n}^{*}-\mathrm{n}$ & 3,4 & 2,3 & 2 & 2 & $2,3,5$ \\
\hline $\mathrm{i}_{\text {short }}$ & 2,3 & $1,5,6$ & 1 & 2,3 & $1,2,7$ \\
\hline $\mathrm{i}_{\text {long }}$ & 3 & $1,4,5$ & 2 & 3 & $1,2,7$ \\
\hline$\Delta \mathrm{w}$ & 3 & - & - & - & - \\
\hline 1 & - & - & - & 5 & - \\
\hline $1+r_{\text {short }}$ & 3 & - & - & 1 & 2 \\
\hline$\Delta \mathrm{p}_{M}$ & 3 & 3,4 & $2-4$ & - & 5 \\
\hline$\Delta \mathrm{p}_{\text {oil }}$ & - & & & & \\
\hline
\end{tabular}

Where INSTPV is the fit of

$$
\frac{(1-0.75)(1-0.99 * 0.75))}{1-(0.99 * 0.75)^{13}} \sum_{i=0}^{12}(0.99 * 0.75)^{i}\left(p_{t+i}^{*}-p_{t-1}\right)
$$

with respect to the lagged values of variables $\mathrm{p}, \mathrm{p}^{*}, n^{*}-n, \Delta \mathrm{p}_{M}, \mathrm{p}_{M}, \mathrm{p}_{X}, \mathrm{p}_{\text {oil }}, \mathrm{i}_{\text {short }}$, $\mathrm{i}_{\text {long }}$ in the interval from minus 1 to minus 12. To retain the degrees of freedom sufficient only the lags, the significance level of which exceeded 5 per cent, were approved.

Normalising the equation with respect to the current period and replacing the time-varying discount factor by its sample average gives the following representation:

FR: $\Delta p_{t}=0.5576 \cdot \Delta p_{t+1}+0.4354 \cdot \Delta p_{t-1}+0.0132 \cdot\left(p_{t}^{*}-p_{t}\right)$

DE: $\Delta p_{t}=0.6612 \cdot \Delta p_{t+1}+0.3346 \cdot \Delta p_{t-1}+0.0308 \cdot\left(p_{t}^{*}-p_{t}\right)$

IT: $\Delta p_{t}=0.5904 \cdot \Delta p_{t+1}+0.4064 \cdot \Delta p_{t-1}+0.0082 \cdot\left(p_{t}^{*}-p_{t}\right)$

$\mathrm{ES}: \Delta p_{t}=0.5909 \cdot \Delta p_{t+1}+0.4050 \cdot \Delta p_{t-1}+0.0504 \cdot\left(p_{t}^{*}-p_{t}\right)$

$\mathrm{NL}: \Delta p_{t}=0.6245 \cdot \Delta p_{t+1}+0.3735 \cdot \Delta p_{t-1}+0.0271 \cdot\left(p_{t}^{*}-p_{t}\right)$

\section{A.4 NKPC wage equation}

\begin{tabular}{|r|c|c|c|c|c|}
\hline & \multicolumn{5}{|c|}{ Lags of Instruments } \\
\hline Instruments & DE & FR & IT & ES & NL \\
\hline$\Delta \mathrm{w}$ & $2,3,4$ & 2 & 2,3 & 2 & 2 \\
\hline $\mathrm{w}_{u \text { uu }}^{*}-\mathrm{w}$ & - & 1,2 & 2 & 2,3 & 1 \\
\hline $\mathrm{w}_{\text {nu }}^{*}-\mathrm{w}$ & - & 2 & 2,3 & 2 & 1,3 \\
\hline $\mathrm{n}^{*}-\mathrm{n}$ & 9,12 & - & 2,3 & 3 & 2 \\
\hline $\mathrm{i}_{\text {short }}$ & $3,7,10$ & 3 & - & 6 & 3,6 \\
\hline $\mathrm{i}_{\text {long }}$ & 3,12 & 3 & 4 & 1 & 1,8 \\
\hline$\Delta \mathrm{p}_{E I}$ & - & 1 & 1 & 5 & 2,3 \\
\hline$\Delta \mathrm{p}_{M}$ & - & 2,3 & 2 & - & - \\
\hline$\Delta \mathrm{p}_{X}$ & - & 2,3 & - & - & - \\
\hline $\mathrm{INSTPV}_{\mathrm{NPV}}$ & 2,3 & - & - & - & - \\
\hline
\end{tabular}


where $\mathrm{w}_{u u}^{*}$ and $\mathrm{w}_{n u}^{*}$ and refer to the optimal frictionless wage rates of the utilitarian and non-utilitarian unions, respectively, and INSTPV is computed with respect to the lagged variables of $\mathrm{w}_{u u}^{*}, \mathrm{w}_{n u}^{*}, \mathrm{w}, \Delta \mathrm{p}$ and $n^{*}-n$ in the interval from minus 1 to minus 12 .

Normalising the equation with respect to the current period and replacing the time-varying discount factor by its sample average gives the following representation:

$\mathrm{FR}: \Delta w_{t}=0.5813 \cdot \Delta w_{t+1}+0.4143 \cdot \Delta w_{t-1}+0.0096 \cdot\left(w_{t}^{*}-w_{t}\right)$

$\mathrm{DE}: \Delta w_{t}=0.63940 \cdot \Delta w_{t+1}+0.3569 \cdot \Delta w_{t-1}+0.0191 \cdot\left(w_{t}^{*}-w_{t}\right)$

IT: $\Delta w_{t}=0.5993 \cdot \Delta w_{t+1}+0.3956 \cdot \Delta w_{t-1}+0.0572 \cdot\left(w_{t}^{*}-w_{t}\right)$

$\mathrm{ES}: \Delta w_{t}=0.5029 \cdot \Delta w_{t+1}+0.4926 \cdot \Delta w_{t-1}+0.0060 \cdot\left(w_{t}^{*}-w_{t}\right)$

$\mathrm{NL}: \Delta w_{t}=0.6338 \cdot \Delta w_{t+1}+0.3672 \cdot \Delta w_{t-1}+0.0129 \cdot\left(w_{t}^{*}-w_{t}\right)$

\section{A.5 Inventory equation}

\begin{tabular}{|r|c|c|c|c|c|}
\hline & \multicolumn{5}{|c|}{ Lags of Instruments } \\
\hline Instruments & $\mathrm{DE}$ & $\mathrm{FR}$ & $\mathrm{IT}$ & $\mathrm{ES}$ & $\mathrm{NL}$ \\
\hline$\Delta \mathrm{KII}$ & $2-5$ & $2-5$ & $2-5$ & 3,5 & $2,3,5$ \\
\hline$\Delta \mathrm{KII}^{*}$ & $2-4$ & $2-4$ & $3-5$ & $3-5$ & $2,4,5$ \\
\hline$\Delta \mathrm{S}$ & 2,3 & 2 & 3 & 3 & 3 \\
\hline $\mathrm{r}_{\text {short }}$ & $2-5$ & $2-5$ & $2-5$ & $2-5$ & $2,3,5$ \\
\hline $\mathrm{n}^{*} \mathrm{n}$ & 2 & 2,3 & 3 & 3 & 2,3 \\
\hline
\end{tabular}

\section{A.6 Consumption equation}

\begin{tabular}{|r|c|c|c|c|c|}
\hline & \multicolumn{5}{|c|}{ Lags of Instruments } \\
\hline Instruments & $\mathrm{DE}$ & $\mathrm{FR}$ & $\mathrm{IT}$ & $\mathrm{ES}$ & $\mathrm{NL}$ \\
\hline$\frac{C}{Y}$ & 2,3 & 3,4 & $2-4$ & $2-4$ & 2,3 \\
\hline$\left(\frac{V t-1}{Y}\right)-1$ & 3,4 & $1-3$ & $1-3$ & 2,3 & 1,2 \\
\hline $\mathrm{r}_{\text {short }}$ & $2,4-6$ & $4-8$ & $3,5,7$ & $4-8$ & $4-7$ \\
\hline $\mathrm{r}_{\text {long }}$ & 2,4 & $2-4$ & $2-4$ & $2-4$ & $2-4$ \\
\hline $\mathrm{URX}$ & & 4 & - & 4 & $2-4$ \\
\hline$\Delta_{4} \mathrm{URX}$ & & 2,3 & 2,3 & - & - \\
\hline$\frac{P_{S}}{P_{I}}$ & $2,3,6$ & - & 6 & 6 & 2 \\
\hline$\frac{P_{H}}{P_{I}}$ & 2 & - & - & 2,3 & 3 \\
\hline$\frac{P_{I}}{P_{C}}$ & 2 & - & 3 & - & 1 \\
\hline$\frac{K H R}{K P}$ & - & - & - & 1 & - \\
\hline
\end{tabular}




\section{B Figures}

Figure 1: Short-term Interest rate shock (50bp)
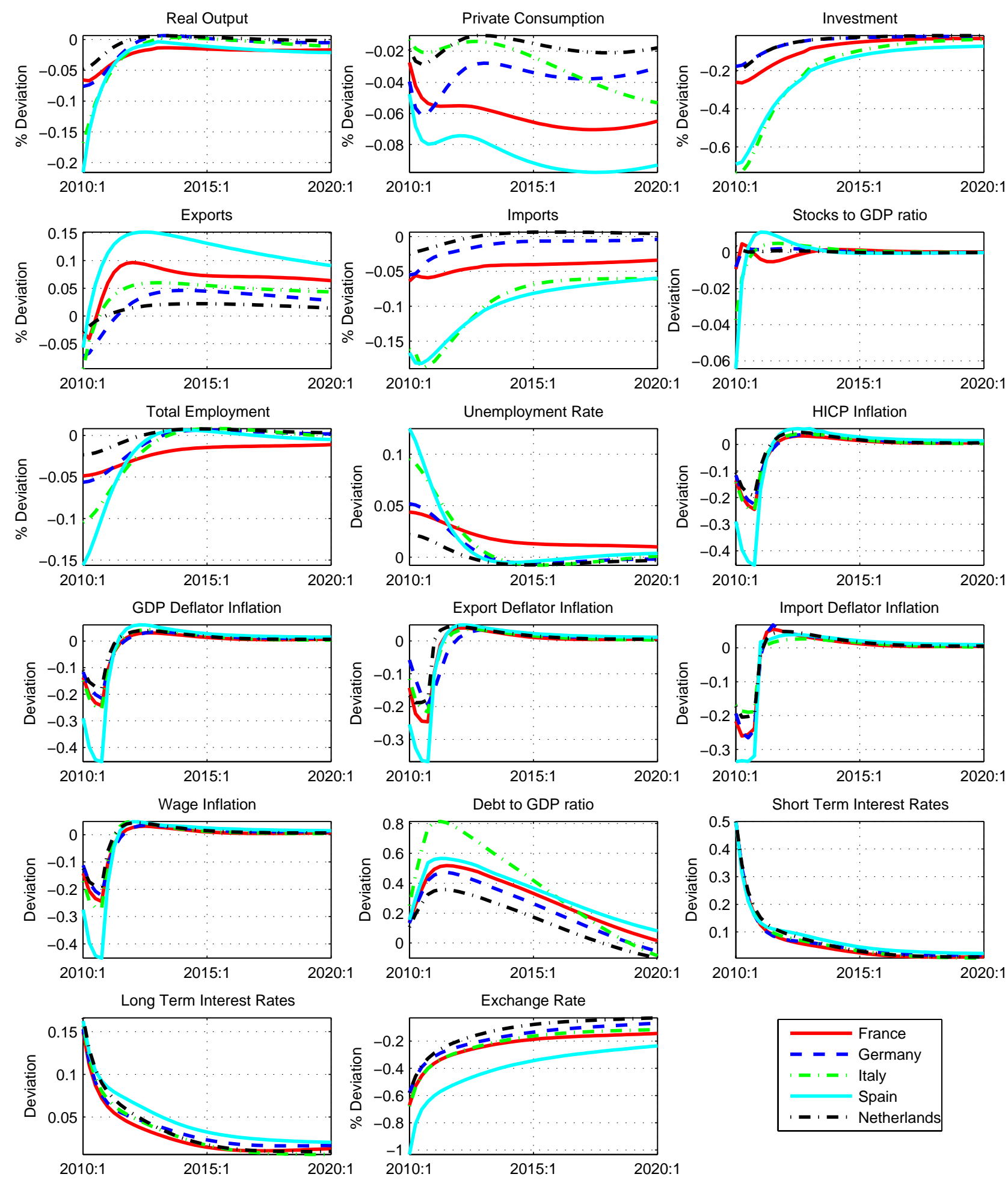

Real variables, employment and exchange rate are presented as percentage deviations from baseline, all other variables are in difference from baseline (either percentage points or percent of GDP). Inflation is computed as year-on-year rate of change. 
Figure 2: Permanent Government Consumption (0.5\% GDP)
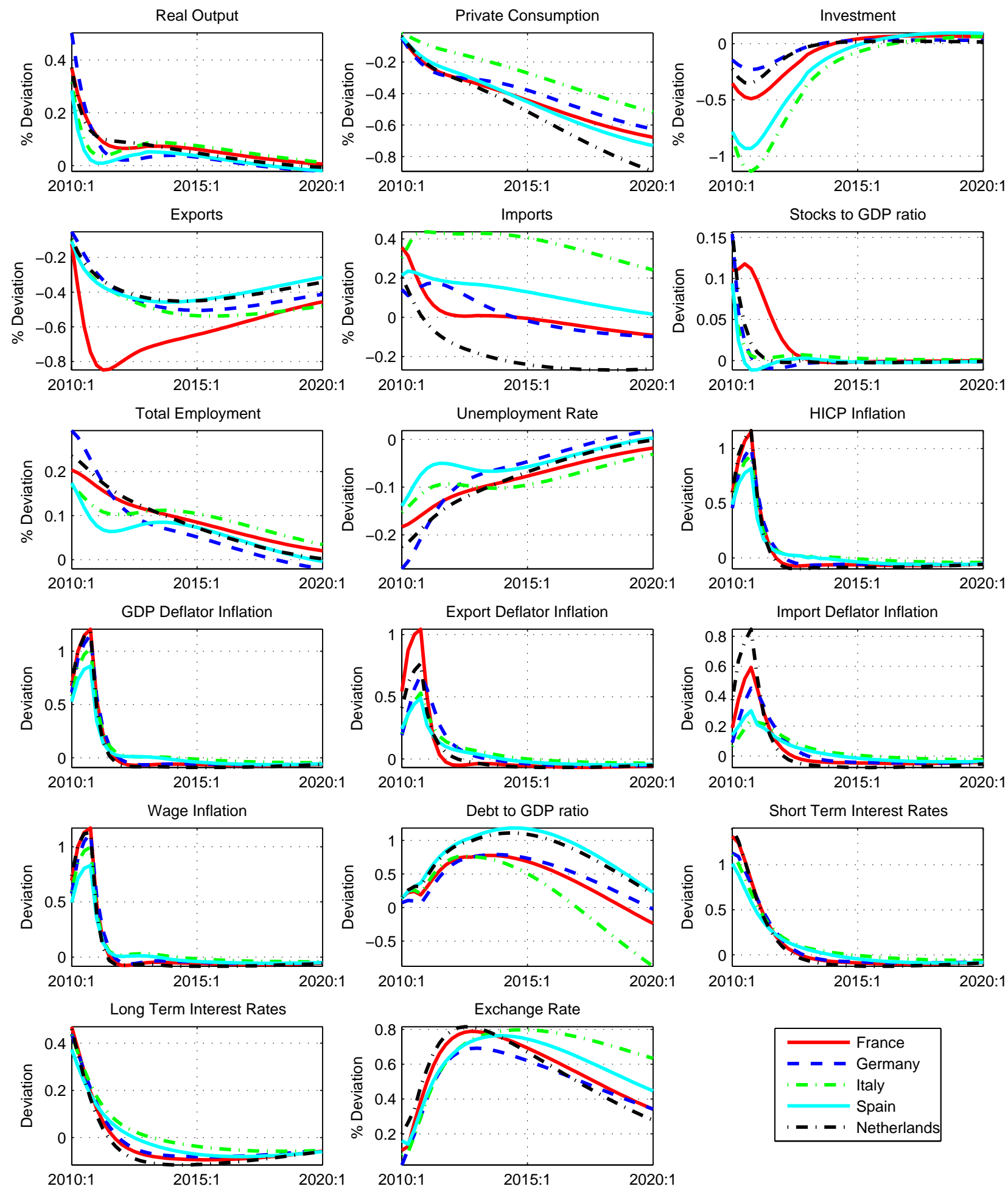

Real variables, employment and exchange rate are presented as percentage deviations from baseline, all other variables are in difference from baseline (either percentage points or percent of GDP). Inflation is computed as year-on-year rate of change. 
Figure 3: Labour Augmenting Technology shock (0.1\% GDP)
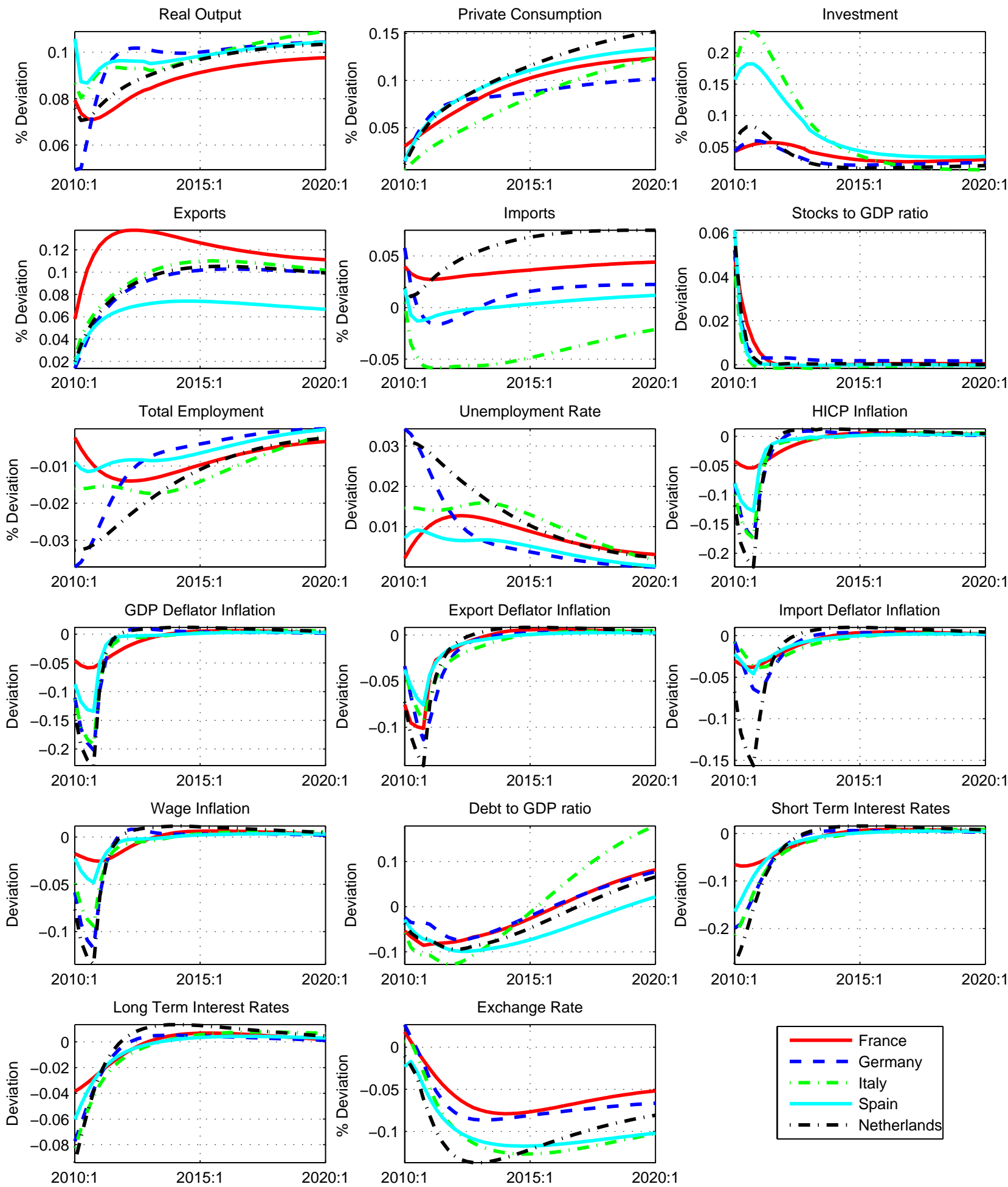

Real variables, employment and exchange rate are presented as percentage deviations from baseline, all other variables are in difference from baseline (either percentage points or percent of GDP). Inflation is computed as year-on-year rate of change. 
Figure 4: Capital Augmenting Technology shock (0.1\% GDP)
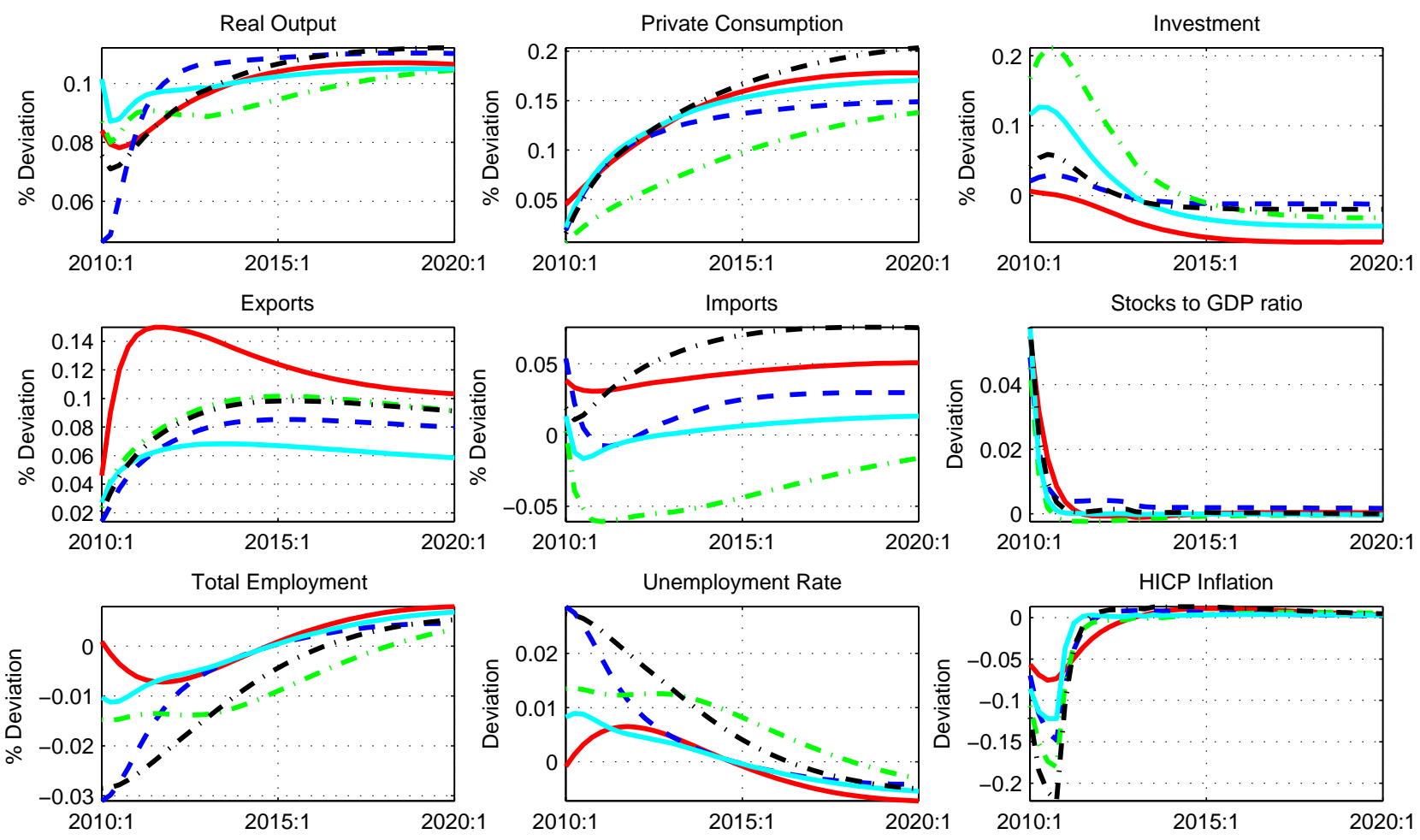

GDP Deflator Inflation
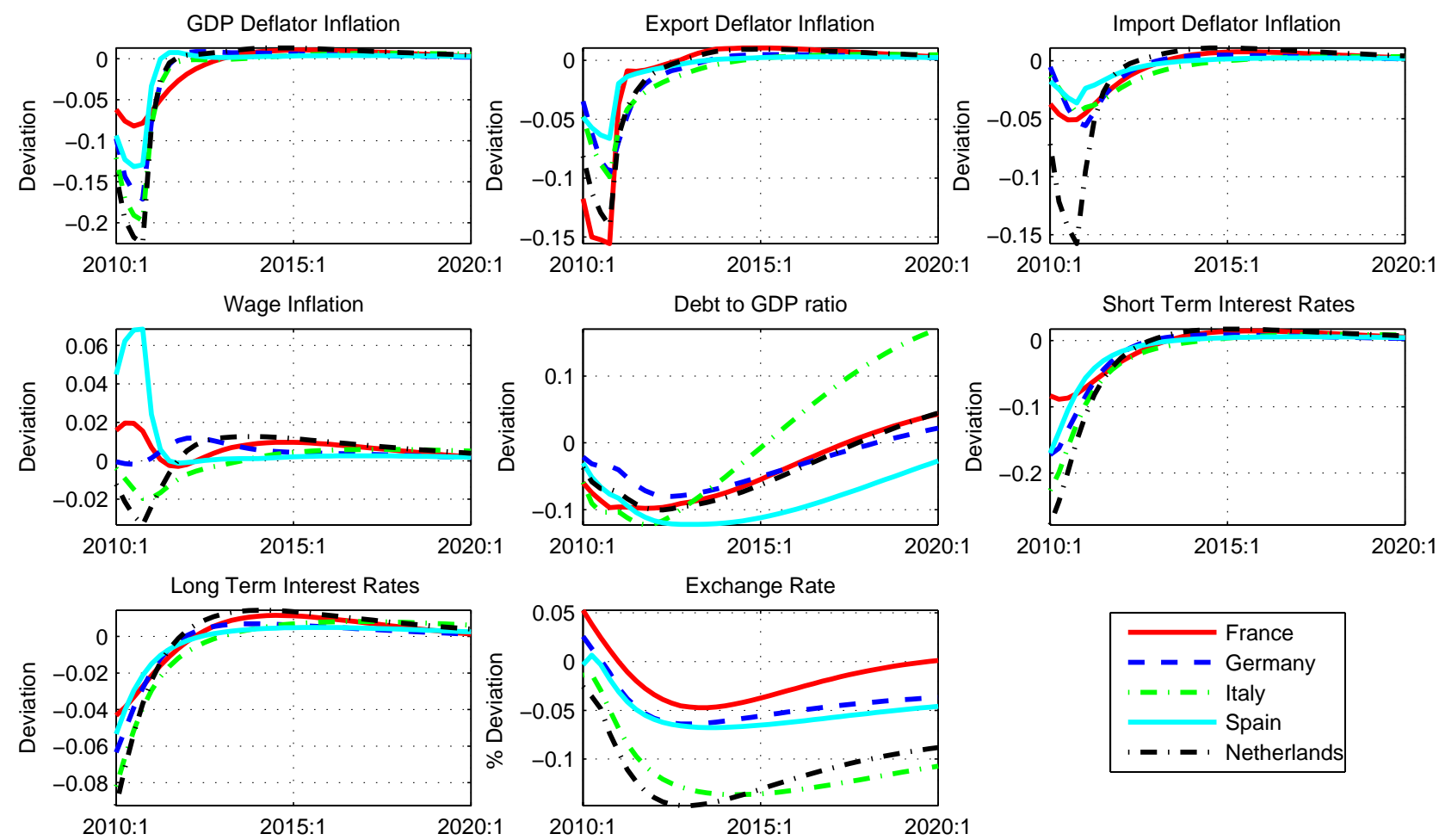

Real variables, employment and exchange rate are presented as percentage deviations from baseline, all other variables are in difference from baseline (either percentage points or percent of GDP). Inflation is computed as year-on-year rate of change. 
Figure 5: Permanent Exchange rate shock (5\%)
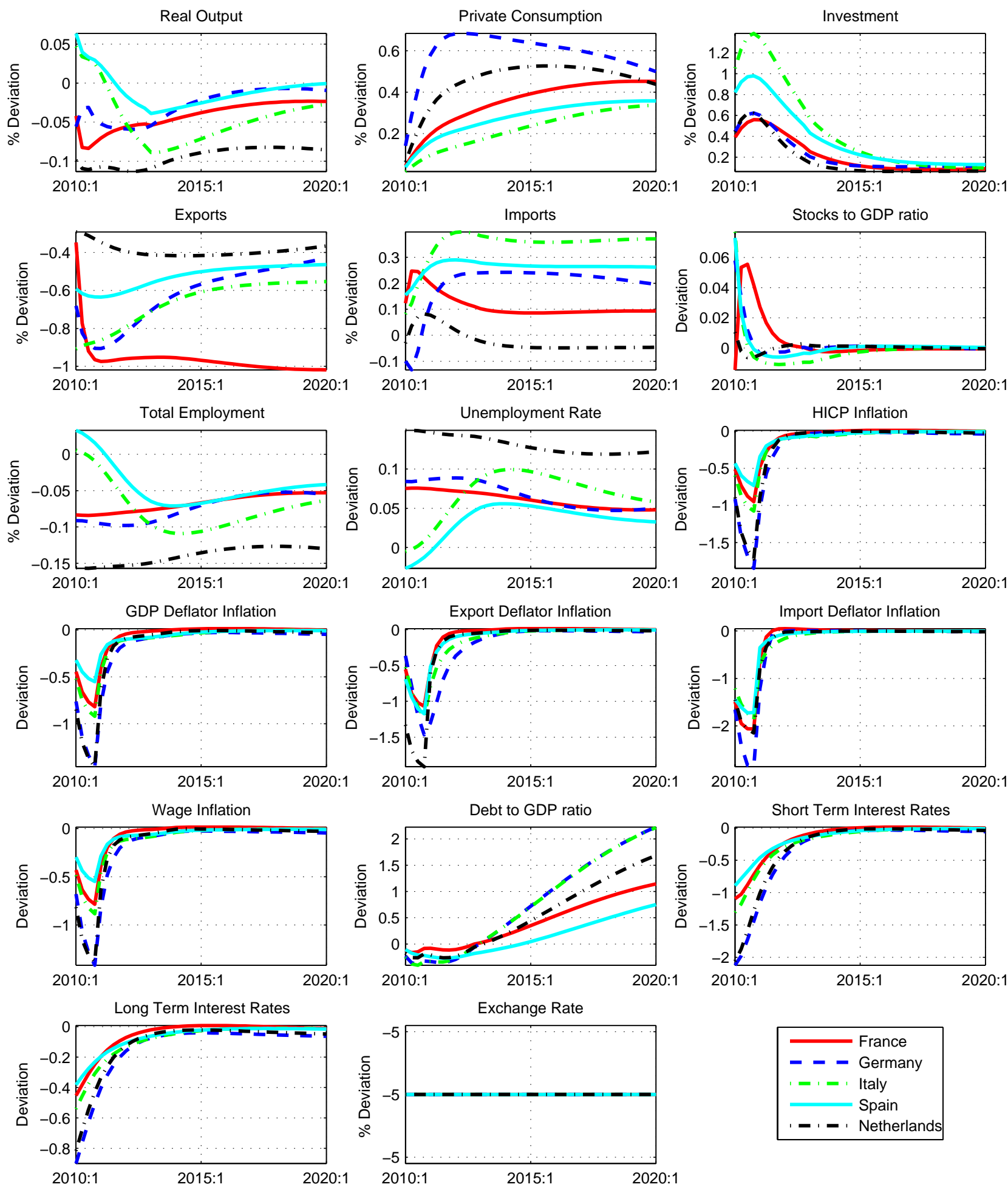

Real variables, employment and exchange rate are presented as percentage deviations from baseline, all other variables are in difference from baseline (either percentage points or percent of GDP). Inflation is computed as year-on-year rate of change. 
Figure 6: Permanent World Demand shock (1\%)
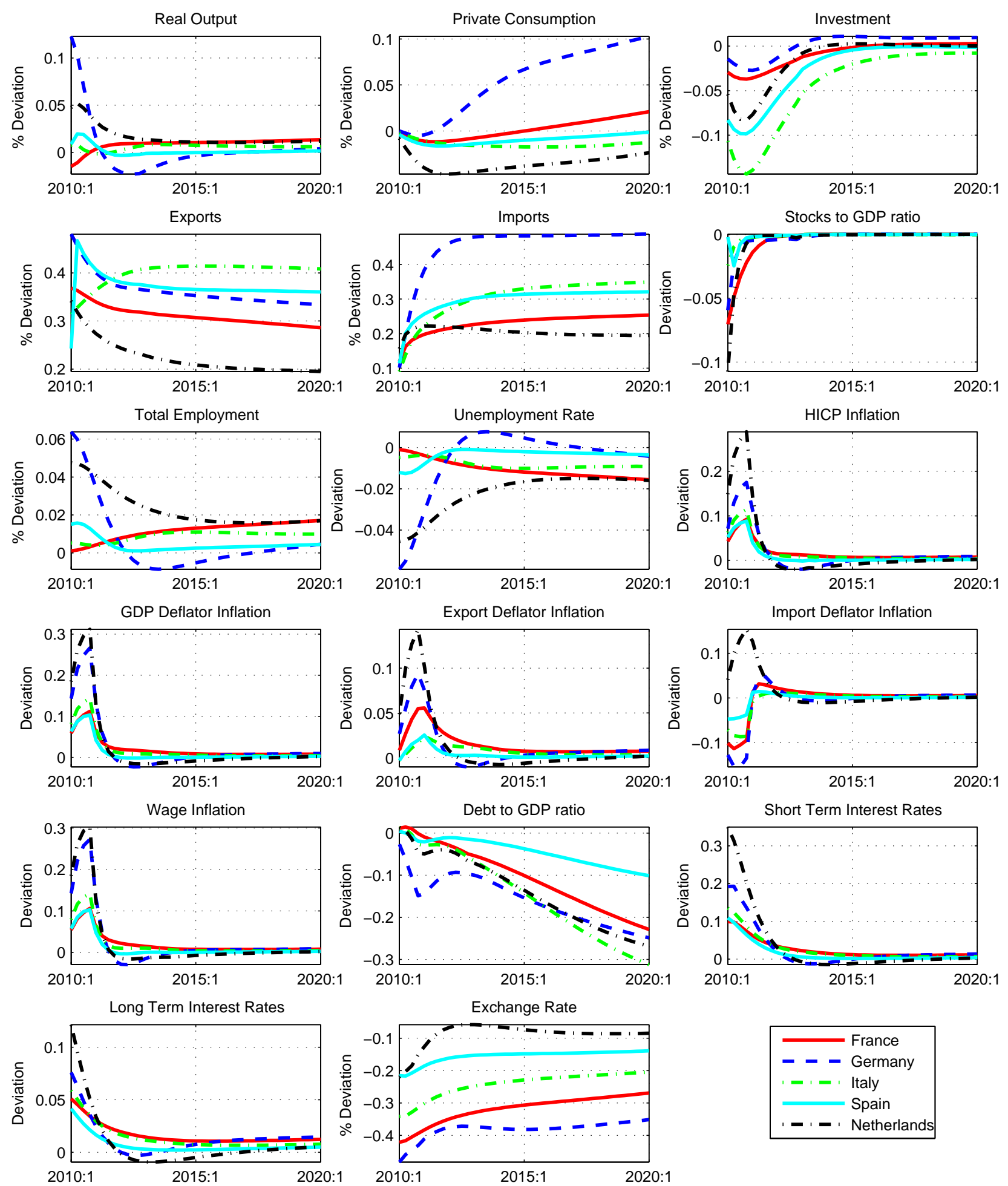

Real variables, employment and exchange rate are presented as percentage deviations from baseline, all other variables are in difference from baseline (either percentage points or percent of GDP). Inflation is computed as year-on-year rate of change. 
Figure 7: Announced and pre-announced shock

Government Consumption ( $0.5 \%$ of GDP) for 3 years - France
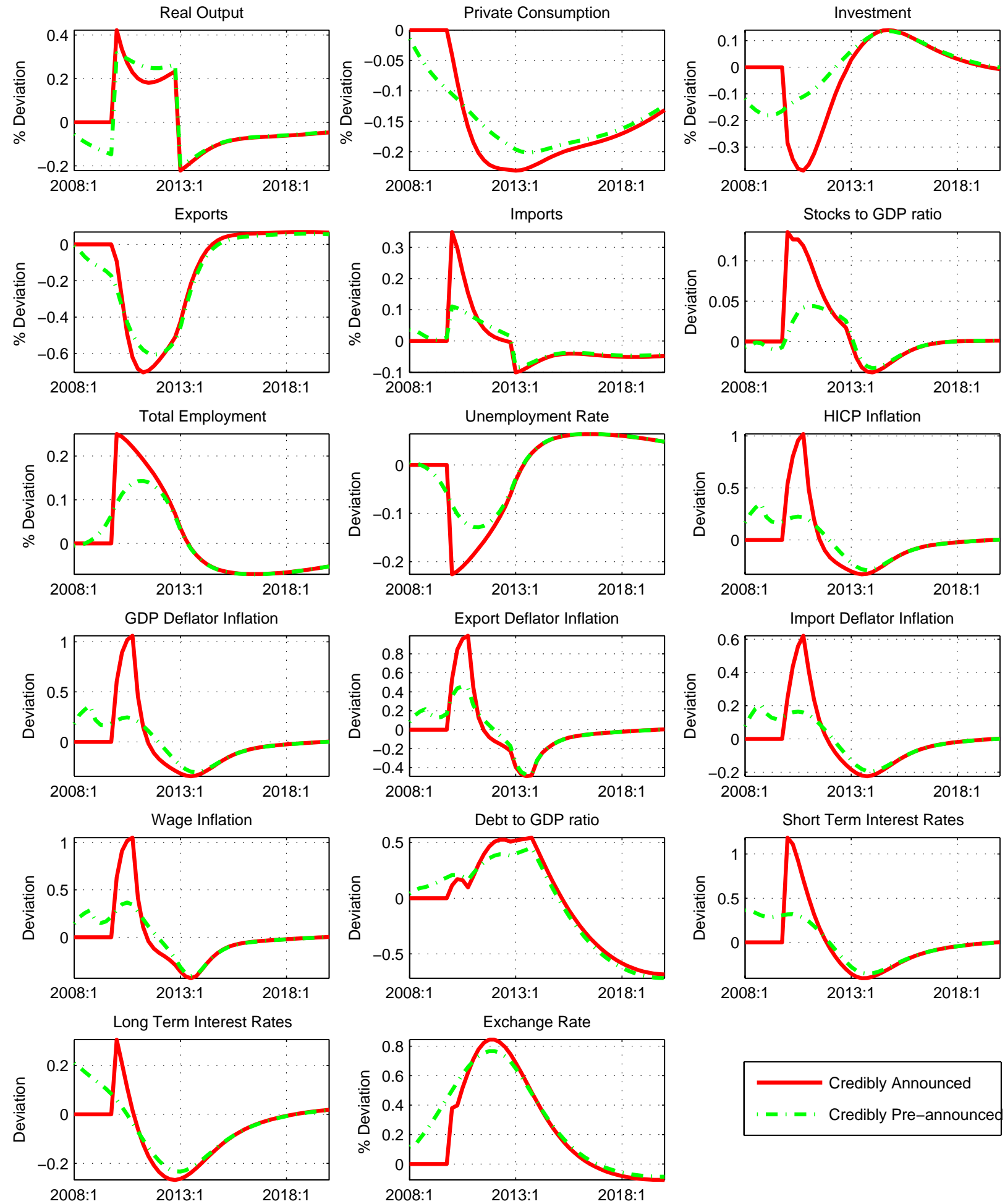

Real variables, employment and exchange rate are presented as percentage deviations from baseline, all other variables are in difference from baseline (either percentage points or percent of GDP). Inflation is computed as year-on-year rate of change. 
Figure 8: Credibly Announced and unannounced / uncredible shock Government Consumption ( $0.5 \%$ of GDP) for 3 years - France
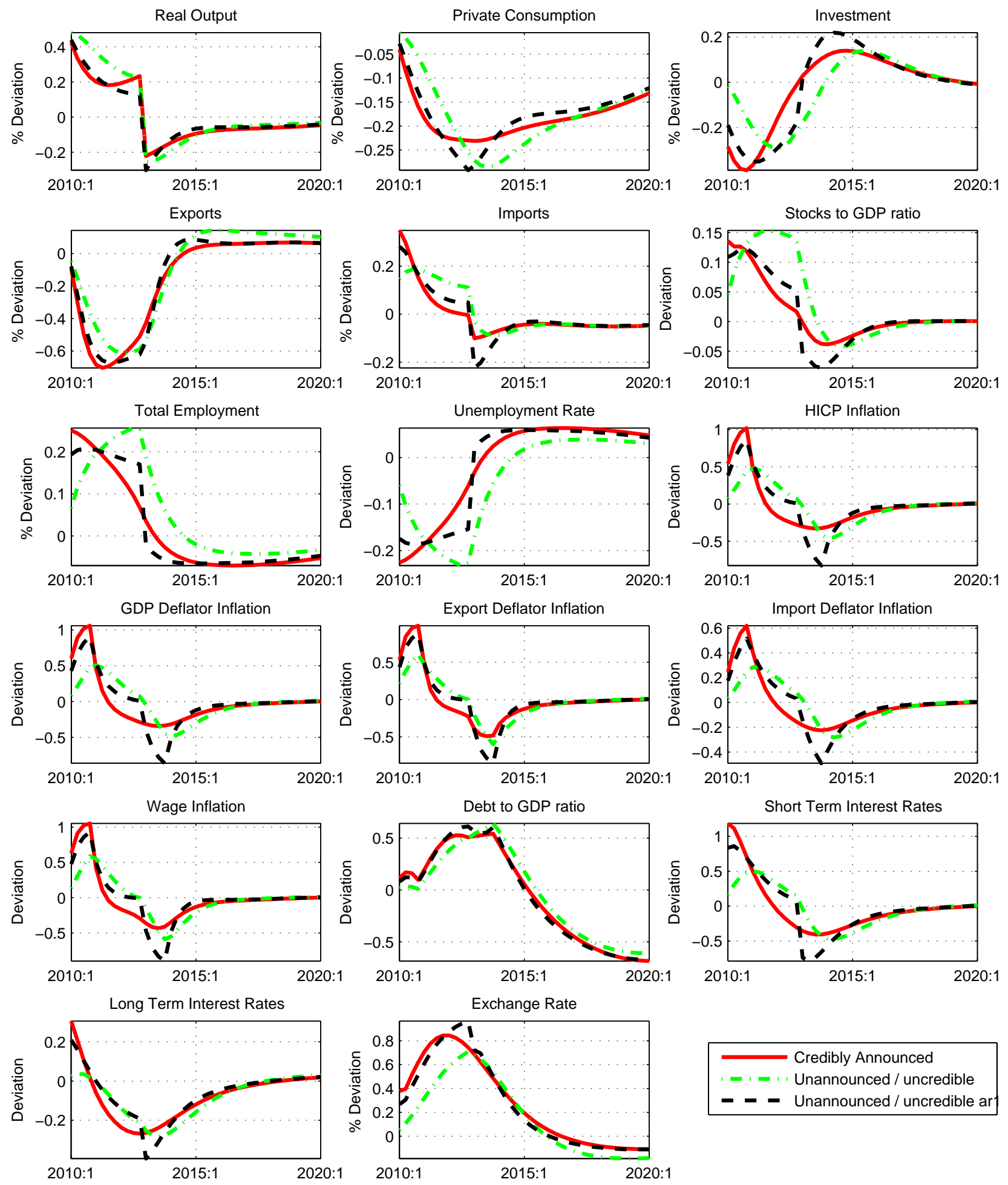

Real variables, employment and exchange rate are presented as percentage deviations from baseline, all other variables are in difference from baseline (either percentage points or percent of GDP). Inflation is computed as year-on-year rate of change. 
